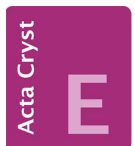

CRYSTALLOGRAPHIC COMMUNICATIONS

ISSN 2056-9890

Received 28 January 2015

Accepted 19 February 2015

Edited by P. C. Healy, Griffith University, Australia

Keywords: crystal structure; metalloligand; manganese; terpyridine

CCDC references: 1050540; 1050539 Supporting information: this article has supporting information at journals.iucr.org/e

\section{Crystal structures of the water and acetone monosolvates of bis[4'-(pyridin-4-yl)-2, $2^{\prime}: 6^{\prime}, \mathbf{2}^{\prime \prime}$ - terpyridine]manganese(II) bis(hexafluorido- phosphate)}

Leandro M. O. Lourenço, Filipe A. Almeida Paz and José A. Fernandes*

CICECO, Chemistry Department, Universidade de Aveiro, Campus Universitário de Santiago, 3810-193 Aveiro, Portugal. *Correspondence e-mail: jafernandes@ua.pt

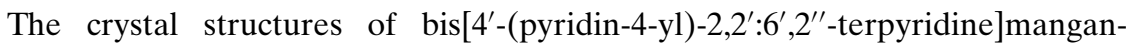
ese(II) bis(hexafluoridophosphate) monohydrate, $\left[\mathrm{Mn}\left(\mathrm{C}_{20} \mathrm{H}_{14} \mathrm{~N}_{4}\right)_{2}\right]\left(\mathrm{PF}_{6}\right)_{2}$--

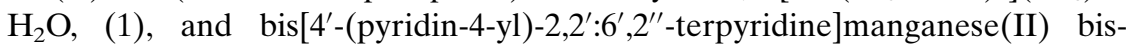
(hexafluoridophosphate) acetone monosolvate, (2), [Mn( $\left.\left.\mathrm{C}_{20} \mathrm{H}_{14} \mathrm{~N}_{4}\right)_{2}\right]$ $\left(\mathrm{PF}_{6}\right)_{2} \cdot \mathrm{CH}_{3} \mathrm{COCH}_{3}$, are described. At $150 \mathrm{~K}$, (1) and (2) have monoclinic $\left(P 2_{1} / c\right)$ and orthorhombic $\left(C 222_{1}\right)$ symmetries, respectively. Both structures exhibit octahedrally coordinated $\mathrm{Mn}^{\mathrm{II}}$ atoms and disorder. They display weak interactions, such as $\mathrm{C}-\mathrm{H} \cdots \mathrm{F}, \mathrm{C}-\mathrm{H} \cdots \mathrm{N}, \mathrm{C}-\mathrm{H} \cdots \pi, \mathrm{F} \cdots \pi$ and $\pi-\pi$. The twofold rotation axis in the molecule of (2) is coincident with a twofold rotation axis of the crystal.

\section{Chemical context}

The synthesis of new metal-organic frameworks (MOFs) can be achieved by several ways with different degrees of reaction control. One way of having a tighter control on the reactions is the use of metalloligands. A metalloligand is a kind of ligand in which the bonding capabilities of the ligand are combined with the directionallity of a metal centre (Halper et al., 2006; Kitagawa et al., 2006; Noro et al., 2005).

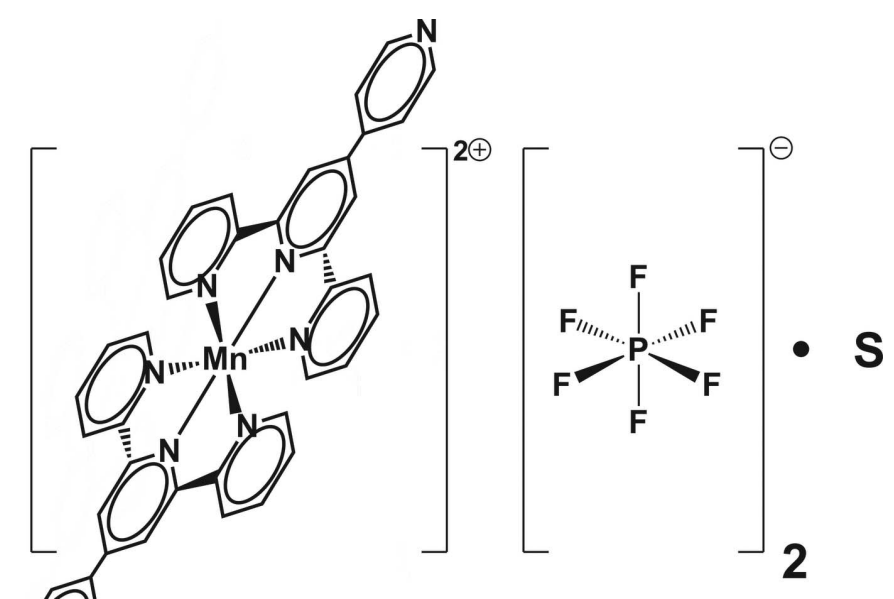

$\mathrm{S}=\mathrm{H}_{2} \mathrm{O}(1)$, acetone (2)

As it is found from a database survey (dedicated section below), there is already a considerable number of compounds of the type $\left[M(\text { Pyterpy })_{2}{ }^{n+}\right]$ [Pyterpy $=4^{\prime}$-(pyridin-4-yl $)-$ 


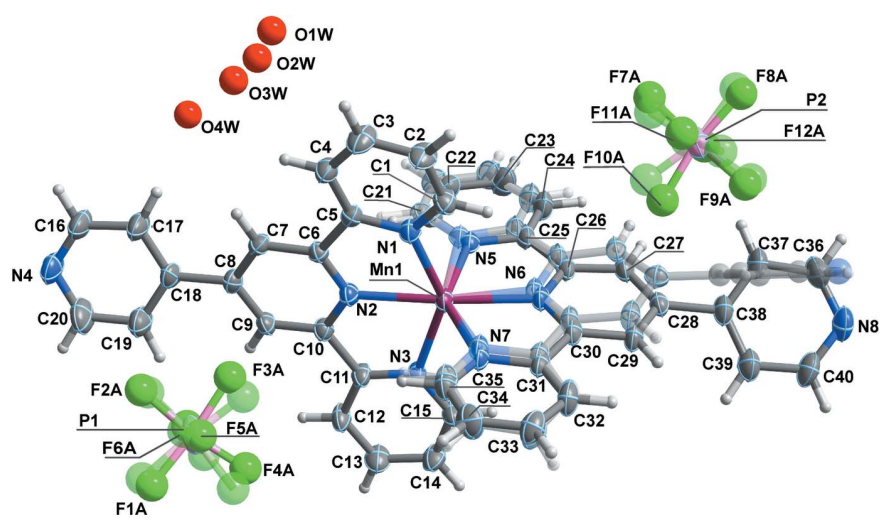

Figure 1

View of the molecular species present in the crystal of (1). Displacement ellipsoids are drawn at the $50 \%$ probability level and the atomic labelling is provided for all non- $\mathrm{H}$ atoms of the components with highest occupancies. Non- $\mathrm{H}$ atoms represented by spheres were isotropically refined and $\mathrm{H}$ atoms are depicted by spheres with arbitrary radius. The componenents with least occupancies are not numbered for the sake of clarity and represented as transparent.

$2,2^{\prime}: 6^{\prime}, 2^{\prime \prime}$-terpyridine]. However, no equivalent structure is found with $\mathrm{Mn}^{2+}$ as metallic centre. In order to fill this gap, we describe in the present report the crystal structure of the water and acetone monosolvates of bis[ $\left[4^{\prime}\right.$-(pyridin-4-yl)-2, $2^{\prime}: 6^{\prime}, 2^{\prime \prime}$ terpyridine]manganese(II) bis(hexafluoridophosphate).

\section{Bis[4'-(pyridin-4-yl)-2, $2^{\prime}: 6^{\prime}, 2^{\prime \prime}$-terpyridine]mangan- ese(II) bis(hexafluoridophosphate) monohydrate, (1)}

\subsection{Structural commentary}

The asymmetric unit of (1) (Fig. 1) comprises a dicationic coordination complex, two highly disordered hexafluoridophosphate charge-balancing anions, and a water molecule distributed among four general locations. The $\mathrm{Mn}^{2+}$ cation is distorted octahedrally coordinated by two $\kappa^{3} N$ - $\left(4^{\prime}\right.$-(pyridin-4yl)-2,2':6', $2^{\prime \prime}$-terpyridine) ligands. One of these ligands is spatially disordered over two close, but distinct, locations, with a distribution of occupancies of 0.85:0.15. Concerning only the major contributor for the disordered ligand, we may say that the coordination environment around the metal cation resembles a highly distorted octahedron with the $\mathrm{Mn}-\mathrm{N}$ distances in the 2.193 (4)-2.268 (4) § range, and the cis and

Table 1

Selected geometric parameters $\left(\AA,{ }^{\circ}\right)$ for $(1)$.

\begin{tabular}{lclc}
\hline $\mathrm{Mn} 1-\mathrm{N} 1$ & $2.231(4)$ & $\mathrm{Mn} 1-\mathrm{N} 5$ & $2.259(5)$ \\
$\mathrm{Mn} 1-\mathrm{N} 2$ & $2.193(4)$ & $\mathrm{Mn} 1-\mathrm{N} 6$ & $2.212(4)$ \\
$\mathrm{Mn} 1-\mathrm{N} 3$ & $2.268(4)$ & $\mathrm{Mn} 1-\mathrm{N} 7$ & $2.260(5)$ \\
& & & \\
$\mathrm{N} 1-\mathrm{Mn} 1-\mathrm{N} 2$ & $72.37(15)$ & $\mathrm{N} 2-\mathrm{Mn} 1-\mathrm{N} 7$ & $117.68(17)$ \\
$\mathrm{N} 1-\mathrm{Mn} 1-\mathrm{N} 3$ & $143.36(15)$ & $\mathrm{N} 3-\mathrm{Mn} 1-\mathrm{N} 5$ & $94.0(3)$ \\
$\mathrm{N} 1-\mathrm{Mn} 1-\mathrm{N} 5$ & $99.8(3)$ & $\mathrm{N} 3-\mathrm{Mn} 1-\mathrm{N} 6$ & $105.2(3)$ \\
$\mathrm{N} 1-\mathrm{Mn} 1-\mathrm{N} 6$ & $111.3(3)$ & $\mathrm{N} 3-\mathrm{Mn} 1-\mathrm{N} 7$ & $96.0(4)$ \\
$\mathrm{N} 1-\mathrm{Mn} 1-\mathrm{N} 7$ & $92.2(4)$ & $\mathrm{N} 5-\mathrm{Mn} 1-\mathrm{N} 6$ & $72.45(17)$ \\
$\mathrm{N} 2-\mathrm{Mn} 1-\mathrm{N} 3$ & $72.21(15)$ & $\mathrm{N} 5-\mathrm{Mn} 1-\mathrm{N} 7$ & $144.44(17)$ \\
$\mathrm{N} 2-\mathrm{Mn} 1-\mathrm{N} 5$ & $97.87(16)$ & $\mathrm{N} 6-\mathrm{Mn} 1-\mathrm{N} 7$ & $71.99(17)$ \\
$\mathrm{N} 2-\mathrm{Mn} 1-\mathrm{N} 6$ & $169.95(18)$ & & \\
\hline
\end{tabular}

Table 2

Hydrogen-bond geometry $\left(\AA,^{\circ}\right)$ for (1).

\begin{tabular}{lllll}
\hline$D-\mathrm{H} \cdots A$ & $D-\mathrm{H}$ & $\mathrm{H} \cdots A$ & $D \cdots A$ & $D-\mathrm{H} \cdots A$ \\
\hline $\mathrm{C} 12-\mathrm{H} 12 \cdots \mathrm{F} 5 A^{\mathrm{i}}$ & 0.95 & 2.50 & $3.422(9)$ & 164 \\
$\mathrm{C} 15-\mathrm{H} 15 \cdots \mathrm{F} 12 A^{\mathrm{ii}}$ & 0.95 & 2.46 & $3.305(9)$ & 149 \\
$\mathrm{C} 16-\mathrm{H} 16 \cdots \mathrm{F} 7 A^{\mathrm{iii}}$ & 0.95 & 2.38 & $3.289(9)$ & 160 \\
$\mathrm{C} 19-\mathrm{H} 19 \cdots \mathrm{F} 5 A^{\mathrm{i}}$ & 0.95 & 2.41 & $3.328(10)$ & 162 \\
$\mathrm{C} 29-\mathrm{H} 29 \cdots \mathrm{N} 4^{\text {iv }}$ & 0.95 & 2.35 & $3.270(8)$ & 163 \\
\hline
\end{tabular}

Symmetry codes: (i) $-x+1,-y+1,-z$; (ii) $x,-y+\frac{1}{2}, z-\frac{1}{2}$; (iii) $x+1, y$, $z$; (iv) $x-1, y, z$.

Table 3

Intramolecular contacts $\left(\AA,^{\circ}\right)$ for $(1)$.

\begin{tabular}{lll}
\hline$D-X \cdots \mathrm{A}$ & $X \cdots \mathrm{A}$ & $D-X \cdots \mathrm{A}$ \\
\hline $\mathrm{C} 13-\mathrm{H} 13 \cdots C g 1^{\mathrm{vi}}$ & 2.83 & 152 \\
$\mathrm{P} 1 A-\mathrm{F} 5 A \cdots C g 2$ & $3.108(8)$ & $129.0(4)$ \\
$\mathrm{P} 2 A-\mathrm{F} 12 A \cdots C g 3^{\text {vii }}$ & $2.906(9)$ & $131.0(4)$ \\
$C g \cdots C g$ & $C g \cdots C g$ & \\
$C g 4 \cdots C g 5$ & $3.779(3)$ & \\
$C g 5 \cdots C g 6^{\mathrm{i}}$ & $3.778(3)$ & \\
\hline
\end{tabular}

Symmetry codes: (i) $-x+1,-y+1,-z$; (vi) $x, \frac{1}{2}-y,-\frac{1}{2}+z$; (vii) $x, \frac{1}{2}-y, \frac{1}{2}+z$. Cg1: centroid of $\{\mathrm{N} 5, \mathrm{C} 21-\mathrm{C} 25\} ; \mathrm{Cg} 2$ : centroid of $\{\mathrm{N} 2, \mathrm{C} 6-\mathrm{C} 10\}$; $C$ g3: centroid of $\{\mathrm{N} 6, \mathrm{C} 26-$ C30\}; $C$ g4: centroid of $\{\mathrm{N} 1, \mathrm{C} 1-\mathrm{C} 5\} ; \mathrm{Cg} 5$ : centroid of $\{\mathrm{N} 3, \mathrm{C} 11-\mathrm{C} 15\} ; \mathrm{Cg} 6$ : centroid of $\{\mathrm{N} 4, \mathrm{C} 16-\mathrm{C} 20\}$.

trans octahedral angles found in the intervals $71.99(17)-$ $117.68(17)$ and $143.36(15)-169.95(18)^{\circ}$, respectively. The angle between the medium planes of the terpyridine moieties is $85.76(14)^{\circ}$, and the angles between the medium planes of non-coordinating pyridines and the terpyridine to which they are attached are 7.9 (2) and 47.1 (3) (see Table 1 for details).

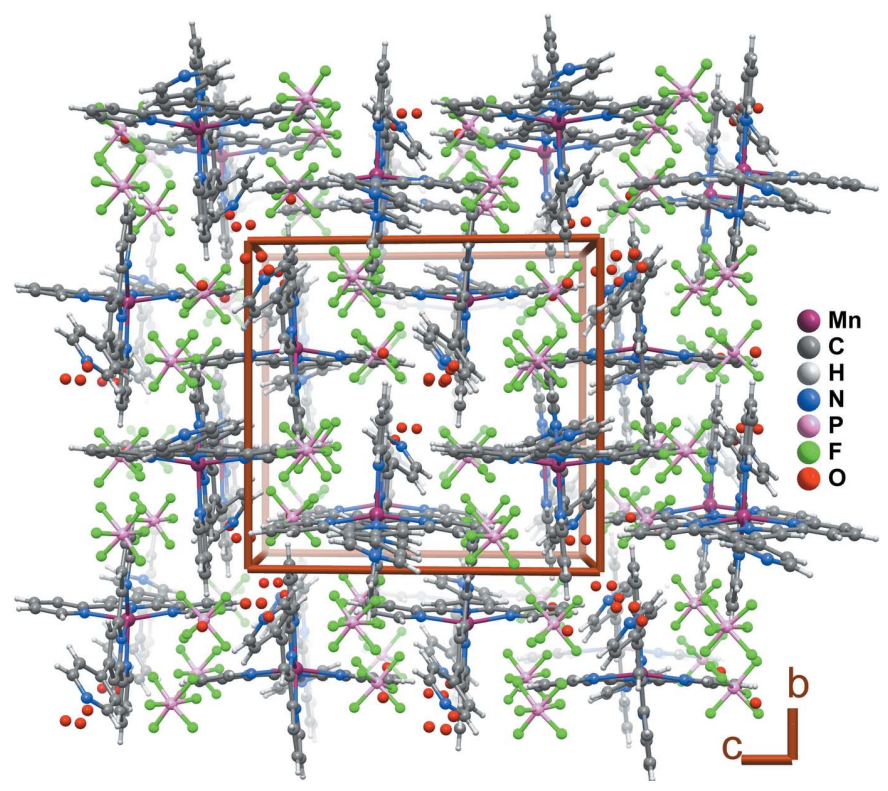

Figure 2

The crystal packing of (1) along the [100] direction. The components for the disordered organic ligand and hexafluoridophosphate with the lowest occupation factors are not represented. Supramolecular interactions are not represented for clarity. 


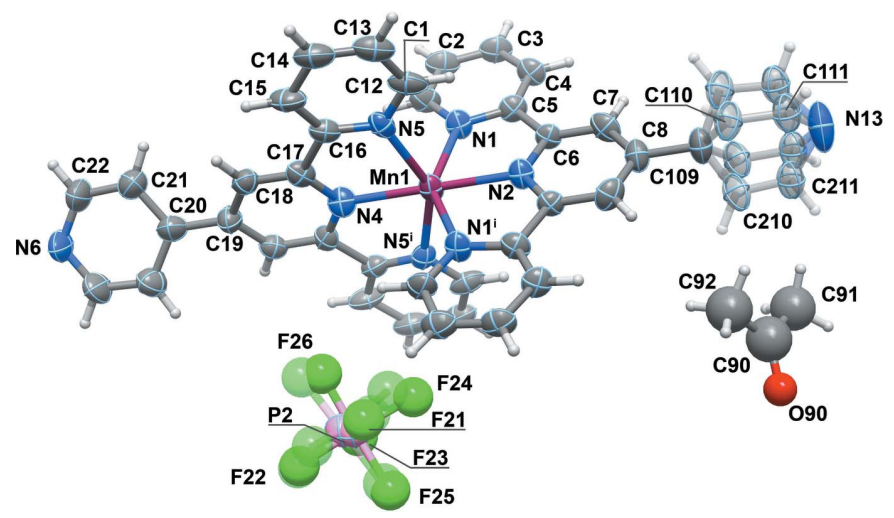

Figure 3

A view of the molecular species present in the crystal of (2). Displacement ellipsoids are drawn at the $50 \%$ probability level and the atomic labelling is provided for all non- $\mathrm{H}$ atoms of the components with highest occupancies. Non-H atoms represented by spheres were isotropically refined and $\mathrm{H}$ atoms are depicted by spheres with arbitrary radius. The componenents with least occupancies are not numbered for the sake of clarity and represented as transparent.

\subsection{Supramolecular features}

The structure of (1) is highly disordered, and the $\mathrm{H}$ atoms belonging to water molecules were not found. For this reason, the description of this structure cannot be thorough (see Fig. 2 for crystal packing). Nevertheless, many weak interactions are present in the crystal structure, namely $\mathrm{C}-\mathrm{H} \cdots \mathrm{F}, \mathrm{C}-\mathrm{H} \cdots \mathrm{N}$, $\mathrm{C}-\mathrm{H} \cdots \pi, \mathrm{F} \cdots \pi$ and $\pi-\pi$ interactions (see Tables 2 and 3 for details). Additionally, there is a close relation between the disordered water molecules and the entities in their neighbourhoods. While the position of site $\mathrm{O} 3 W$ (occupancy 0.15) is incompatible with the position of $\mathrm{N} 8$ (occupancy 0.85) at 2.43 (3) $\AA$, it is foreseeable that it donates a proton to N108 at 2.94 (3) $\AA$. The position of $\mathrm{O} 4 W$ (occupancy 0.85) is also incompatible with the $\mathrm{H} 123$ position [occupancy 0.15 , distance 1.95 (5) $\AA$ ]. Other contact distances are in the limit of possible hydrogen-bonding interactions, namely $\mathrm{O} 1 W \cdots \mathrm{F} 6 B$ [occupancies 0.4 and 0.3333 , distance 2.57 (2) $\AA$ ] , O4W...F3A [occupancies 0.1 and 0.6667 , distance $2.63(5) \AA$ ] and $\mathrm{O} 4 W \cdot \mathrm{H} 23$ [occupancies 0.1 and 0.85 , distance 2.35 (5) $\AA$ ] These relations suggest the water molecule accomodates in the empty spaces left by the disordered organic moieties, or vice versa.

Table 4

Selected geometric parameters $\left(\AA,^{\circ}\right)$ for $(2)$.

\begin{tabular}{lrlr}
\hline Mn1-N1 & $2.210(10)$ & $\mathrm{Mn} 1-\mathrm{N} 4$ & $2.187(14)$ \\
Mn1-N2 & $2.180(13)$ & $\mathrm{Mn} 1-\mathrm{N} 5$ & $2.247(11)$ \\
& & & \\
$\mathrm{N} 1-\mathrm{Mn} 1-\mathrm{N} 1^{\mathrm{i}}$ & $144.8(5)$ & $\mathrm{N} 2-\mathrm{Mn} 1-\mathrm{N} 4$ & 180.0 \\
$\mathrm{~N} 1-\mathrm{Mn} 1-\mathrm{N} 2$ & $72.4(3)$ & $\mathrm{N} 2-\mathrm{Mn} 1-\mathrm{N} 5$ & $107.7(3)$ \\
$\mathrm{N} 1-\mathrm{Mn} 1-\mathrm{N} 4$ & $107.6(3)$ & $\mathrm{N} 4-\mathrm{Mn} 1-\mathrm{N} 5$ & $72.3(3)$ \\
$\mathrm{N} 1-\mathrm{Mn} 1-\mathrm{N} 5$ & $93.5(4)$ & $\mathrm{N} 5-\mathrm{Mn} 1-\mathrm{N} 5^{\mathrm{i}}$ & $144.7(5)$ \\
$\mathrm{N} 1-\mathrm{Mn} 1-\mathrm{N} 5^{\mathrm{i}}$ & $97.0(4)$ & & \\
\hline
\end{tabular}

Symmetry code: (i) $-x+1, y,-z+\frac{3}{2}$.
Table 5

Hydrogen-bond geometry $\left(\AA,^{\circ}\right)$ for (2).

\begin{tabular}{lllll}
\hline$D-\mathrm{H} \cdots A$ & $D-\mathrm{H}$ & $\mathrm{H} \cdots A$ & $D \cdots A$ & $D-\mathrm{H} \cdots A$ \\
\hline $\mathrm{C} 12-\mathrm{H} 12 \cdots \mathrm{F} 25^{\mathrm{ii}}$ & 0.95 & 2.31 & $3.23(3)$ & 162 \\
$\mathrm{C} 15-\mathrm{H} 15 \cdots \mathrm{O} 90^{\mathrm{ii}}$ & 0.95 & 2.58 & $3.53(3)$ & 177 \\
$\mathrm{C} 18-\mathrm{H} 18 \cdots \mathrm{O} 90^{\mathrm{iii}}$ & 0.95 & 2.57 & $3.50(2)$ & 168 \\
$\mathrm{C} 18-\mathrm{H} 18 \cdots \mathrm{O} 90^{\mathrm{ii}}$ & 0.95 & 2.53 & $3.47(2)$ & 169 \\
$\mathrm{C} 22-\mathrm{H} 22 \cdots \mathrm{F} 21^{\mathrm{iv}}$ & 0.95 & 2.47 & $3.42(2)$ & 175 \\
\hline
\end{tabular}

Symmetry codes: (ii) $x-\frac{1}{2},-y+\frac{3}{2},-z+1$; (iii) $x-\frac{1}{2}, y+\frac{1}{2}, z$; (iv) $-x+1,-y+2$, $z+\frac{1}{2}$.

Table 6

Intramolecular contacts $\left(\AA,^{\circ}\right)$ for $(2)$.

\begin{tabular}{lll}
\hline$D-X \cdots \mathrm{A}$ & $X \cdots \mathrm{A}$ & $D-X \cdots \mathrm{A}$ \\
\hline $\mathrm{C} 2-\mathrm{H} 2 \cdots C g 1^{\mathrm{v}}$ & 2.72 & 156 \\
$\mathrm{P} 2-\mathrm{F} 25 \cdots C g 2^{\mathrm{vi}}$ & $3.091(18)$ & $153.0(10)$ \\
$C g \cdots C g$ & $C g \cdots C g$ & \\
$C g 3 \cdots C g 3^{\mathrm{vii}}$ & $3.539(7)$ & \\
\hline
\end{tabular}

Symmetry codes: (v) $x, y, z+1 ;$ (vi) $-x+\frac{3}{2},-y+\frac{3}{2}, z-\frac{1}{2}$; (vii) $-x+1, y,-z+\frac{5}{2}$. Cg1: centroid of $\{\mathrm{N} 5, \mathrm{C} 12-\mathrm{C} 16\} ; \mathrm{Cg} 2$ : centroid of $\left\{\mathrm{N} 2, \mathrm{C} 6-\mathrm{C} 8, \mathrm{C}^{\mathrm{i}}, \mathrm{C} 7\right\}$; $C \mathrm{Cg} 3$ : centroid of $\{\mathrm{N} 1$, $\mathrm{C} 1-\mathrm{C} 5\}$

3. Bis[4'-(pyridin-4-yl)-2, $2^{\prime}: 6^{\prime}, 2^{\prime \prime}$-terpyridine]manganese(II) bis(hexafluoridophosphate) acetone monosolvate, (2)

\subsection{Structural commentary}

Except for the type of the co-crystallizing solvent, compound (2) (Fig. 3) is very similar to (1). However, the molecule of (2) exhibits a twofold rotation axis which is coincident to the axis of the coordination complex, which passes through atoms N13, C109, C8, N2, Mn1, N4, C19, C20 and N6. Thus, the asymmetric unit comprises one half of the

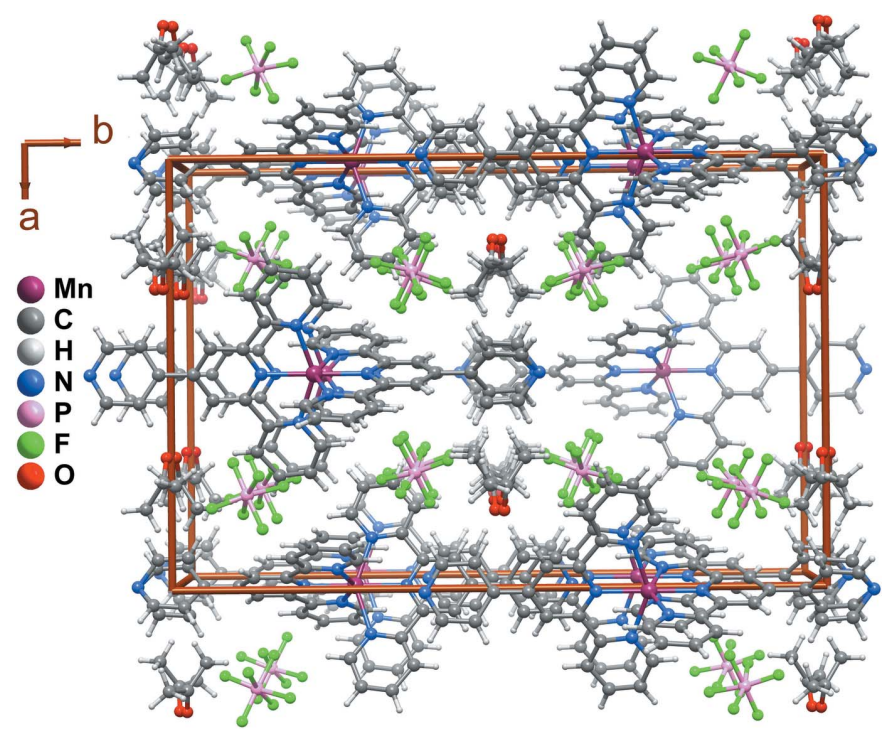

Figure 4

The crystal packing of (2) along the [001] direction. The components for the disordered organic ligand and hexafluoridophosphate with the lowest occupation factors are not represented. Supramolecular interactions are not represented for clarity. 
Table 7

Known structures of the type $\left[M(\text { Pyterpy })_{2}\right]^{n+}\left(X^{-}\right)_{n}$.

\begin{tabular}{|c|c|c|c|c|}
\hline Metal Center & Anion & CCDC code & Reference & Space Group \\
\hline $\mathrm{Fe}^{2+}$ & $\mathrm{PF}_{6}^{-}$ & KITFEZ & Beves, Dunphy, et al. (2008) & $I 4_{1} / a$ \\
\hline $\mathrm{Fe}^{2+}$ & $\mathrm{ClO}_{4}^{-}$ & OFUKAC & Beves, Bray et al. (2008) & $P \overline{1}$ \\
\hline $\mathrm{Fe}^{2+}$ & $\mathrm{SCN}^{-}$ & UGEKEX & Morsali et al. (2009) & $P \overline{1}$ \\
\hline $\mathrm{Fe}^{2+}$ & $\mathrm{ClO}_{4}^{-}$ & XIQFEJ & (a) & $P 2_{1} / c$ \\
\hline $\mathrm{Fe}^{2+}$ & {$\left[\mathrm{Fe}(\mathrm{SCN})_{6}\right]^{3-}$} & XIQFIN & (a) & Pbcn \\
\hline $\mathrm{Fe}^{2+}$ & {$\left[\mathrm{Fe}(\mathrm{SCN})_{6}\right]^{3-}$} & XISWUS & (a) & Pbcn \\
\hline $\mathrm{Co}^{2+}$ & $\mathrm{NO}_{3}^{-}$ & VEYGIQ & Indumathy et al. (2007) & $P \overline{1}$ \\
\hline $\mathrm{Cu}^{2+}$ & $\mathrm{PF}_{6}^{-}$ & FIYHIF & Pitarch López et al. (2005) & $P 4_{1}$ \\
\hline $\mathrm{Zn}^{2+}$ & $\mathrm{NO}_{3}^{-}$ & ULAFET & Ding et al. (2009) & $\mathrm{P}_{3}$ \\
\hline $\mathrm{Zn}^{2+}$ & $\mathrm{ClO}_{4}^{-}$ & BIGDEC & Mehrani et al. (2013) & $C 2 / c$ \\
\hline $\mathrm{Ru}^{2+}$ & $\mathrm{PF}_{6}^{-}$ & OFUKEG & Beves, Bray et al. (2008) & $I 4_{1} / a$ \\
\hline $\mathrm{Ru}^{2+}$ & $\mathrm{PF}_{6}^{-} / \mathrm{NO}_{3}{ }^{-}$ & OFUKIK & Beves, Bray et al. (2008) & $P \overline{1}$ \\
\hline $\mathrm{Ru}^{2+}$ & {$\left[\mathrm{Fe}(\mathrm{SCN})_{6}\right]^{3-}$} & OFUKOQ & Beves, Bray et al. (2008) & $C 2 / c$ \\
\hline $\mathrm{Ru}^{2+}$ & $\mathrm{PF}_{6}^{-} / \mathrm{NO}_{3}^{-}$ & PEHPOI & Constable et al. (2006) & $P 2_{1} / c$ \\
\hline
\end{tabular}

(a) Beves, Constable, Housecroft, Kepert, Neuburger et al. (2007).

dicationic coordination compound, one disordered charge balancing hexafluoridophosphate anion and half of an acetone molecule. In the cation, only one of the noncoordinating pyridine moieties is affected by disorder. Nevertheless, the geometrical environment around the metal cation is still a distorted octahedron (with a symmetry axis in one of the diagonals), with $\mathrm{Mn}-\mathrm{N}$ distances in the $2.180(13)-$ 2.247 (11) $\AA$ range, and the cis and trans octahedral angles in the intervals $72.3(3)-107.7$ (5) and 144.7 (5)-180.0 $0^{\circ}$, respectively. The angle between the medium planes of the terpyridine moieties is $89.5(3)^{\circ}$, and the angles between the medium planes of non-coordinating pyridines and the terpyridine to which they are attached are 35.3 (non-disordered), 62.3 (12) and $65.8(13)^{\circ}$ (disordered) (see Table 4 for details).

\subsection{Supramolecular features}

Similarly to (1), in the structure of compound (2) (Fig. 4) there are a considerable number of weak interactions present, namely $\mathrm{C}-\mathrm{H} \cdots \mathrm{F}, \mathrm{C}-\mathrm{H} \cdots \mathrm{O}, \mathrm{C}-\mathrm{H} \cdots \pi, \mathrm{F} \cdots \pi$ and $\pi-\pi$ interactions (see Tables 5 and 6 for details).

\section{Database survey}

The use of the ligand Pyterpy [described by Constable et al. (2000)] as precursor of metalloligands has contributed for the synthesis of several complexes of the form $\left[M(\text { Pyterpy })_{2}\right]^{n+}\left(X^{-}\right)_{n}$. The metal centres comprise metals with valence +2 of the first transition period, from $\mathrm{Fe}^{2+}$ to $\mathrm{Zn}^{2+}$, as well as $\mathrm{Co}^{3+}, \mathrm{Ru}^{2+}$ and $\mathrm{Rh}^{3+}$ (Groom \& Allen, 2014). The charge-balancing anions are $\mathrm{PF}_{6}{ }^{-}, \mathrm{NO}_{3}{ }^{-}, \mathrm{ClO}_{4}{ }^{-}, \mathrm{SCN}^{-}$or $\left[\mathrm{Fe}(\mathrm{SCN})_{6}\right]^{3-}$ (Beves, Bray et al., 2008; Beves, Constable, Housecroft, Kepert, Neuburger et al., 2007; Beves, Dunphy et al., 2008; Constable et al., 2000, 2006; Ding et al., 2009; Indumathy et al., 2007; Mehrani et al., 2013; Morsali et al., 2009; Paul et al., 2004; Pitarch López et al., 2005; see Table 7 for details).
All the cations exhibit a distorted octahedral geometry, with the Pyterpy ligands in a meridional coordination. Some of the crystal structures sharing the same anion are isotypical. This is the case of the nitrate-containing crystals of $\mathrm{Fe}^{2+}, \mathrm{Co}^{2+}$ and $\mathrm{Ni}^{2+}$ [Cambridge Structural Database (CSD; Groom \& Allen, 2014) refcodes WOMXAX, VEYGIQ and OFUJUV] or the hexafluoridophosphate-containing crystals of $\mathrm{Fe}^{2+}$ and $\mathrm{Ru}^{2+}$ (OFUKEG and KITFEZ). Two different solvates of [Fe(Pyterpy)(PyterpyH)][Fe(SCN) 6 are also isotypical (XIQFIN and XIQFEJ). Additionally, two structures of $\mathrm{Fe}^{2+}$ and $\mathrm{Ru}^{2+}$ have similar cell parameters, despite of not sharing the same anion (OFUKIK and UGEKEX). None of compounds described in this work is isotypical with a previously reported structure.

Until now the use of the metalloligand $\left[M(\text { Pyterpy })_{2}{ }^{n+}\right]$ is still very limited. Some one-dimensional polymers are known (Beves, Constable et al., 2008; Yoshida et al., 2009; Beves,

Table 8

Uses of $\left[M(\text { Pyterpy })_{2}\right]^{n+}$ as metalloligand.

\begin{tabular}{|c|c|c|c|c|}
\hline Metal 1 & Metal 2 & CCDC code & Type $\dagger$ & Reference \\
\hline $\mathrm{Fe}^{2+}$ & $\mathrm{Cu}^{2+}$ & GIVDEV & polymer & $\begin{array}{l}\text { Beves, Constable et al. } \\
\quad(2008)\end{array}$ \\
\hline $\mathrm{Fe}^{2+}$ & $\mathrm{Cu}^{2+}$ & OGOTEK & 5 (linear) & Beves et al. (2009) \\
\hline $\mathrm{Ni}^{2+}$ & $\mathrm{Co}^{2+}$ & WUTTEL & polymer & Yoshida et al. (2009) \\
\hline $\mathrm{Ni}^{2+}$ & $\mathrm{Co}^{2+}$ & WUTTIP & polymer & Yoshida et al. (2009) \\
\hline $\mathrm{Co}^{2+}$ & $\mathrm{Co}^{2+}$ & WUTTOP & polymer & Yoshida et al. (2009) \\
\hline $\mathrm{Ni}^{2+}$ & $\operatorname{Ir}^{3+}$ & MITQUD & 6 (cyclic) & Liu et al. (2014) \\
\hline $\mathrm{Ni}^{2+}$ & $\mathrm{Rh}^{3+}$ & MITRAK & 6 (cyclic) & Liu et al. (2014) \\
\hline $\mathrm{Cu}^{2+}$ & $\mathrm{Ir}^{3+}$ & MITCEZ & 6 (cyclic) & Liu et al. (2014) \\
\hline $\mathrm{Cu}^{2+}$ & $\mathrm{Rh}^{3+}$ & MITCID & 6 (cyclic) & Liu et al. (2014) \\
\hline $\mathrm{Zn}^{2+}$ & $\mathrm{Ir}^{3+}$ & MITQEN & 6 (cyclic) & Liu et al. (2014) \\
\hline $\mathrm{Zn}^{2+}$ & $\mathrm{Ir}^{3+}$ & MITQIR & 6 (cyclic) & Liu et al. (2014) \\
\hline $\mathrm{Zn}^{2+}$ & $\mathrm{Rh}^{3+}$ & MITQOX & 6 (cyclic) & Liu et al. (2014) \\
\hline $\mathrm{Ni}^{2+}$ & $\mathrm{Rh}^{3+}$ & MITCOJ & 3 (linear) & Liu et al. (2014) \\
\hline $\mathrm{Ru}^{2+}$ & $\mathrm{Ag}^{+}$ & WICSIL & polymer & (b) \\
\hline
\end{tabular}

Notes: 'Metal 1' coordinated by Pyterpy; 'Metal 2' bridging metal. $\dagger$ Polymer or number of metals in the oligomer and respective arrangement; $(b)$ Beves, Constable, Housecroft, Kepert, Price et al. (2007). 
Table 9

Experimental details.

Crystal data

Chemical formula

$M_{\mathrm{r}}$

Crystal system, space group

Temperature (K)

$a, b, c(\AA)$

$\alpha, \beta, \gamma\left({ }^{\circ}\right)$

$V\left(\AA^{3}\right)$

$Z$

Radiation type

$\mu\left(\mathrm{mm}^{-1}\right)$

Crystal size ( $\mathrm{mm})$

Data collection

Diffractometer

Absorption correction

$T_{\min }, T_{\max }$

No. of measured, independent and observed

$[I>2 \sigma(I)]$ reflections

$R_{\text {int }}$

$(\sin \theta / \lambda)_{\max }\left(\AA^{-1}\right)$

Refinemen

$R\left[F^{2}>2 \sigma\left(F^{2}\right)\right], w R\left(F^{2}\right), S$

No. of reflections

No. of parameters

No. of restraints

$\mathrm{H}$-atom treatment

$\Delta \rho_{\max }, \Delta \rho_{\min }\left(\mathrm{e} \AA^{-3}\right)$

Absolute structure

Absolute structure parameter

Computer programs: APEX2 and SAINT (Bruker, 2007), SHELXS97, SHELXL97 and SHELXTL (Sheldrick, 2008), SHELXL2014 (Sheldrick, 2015).
(1)

(2)

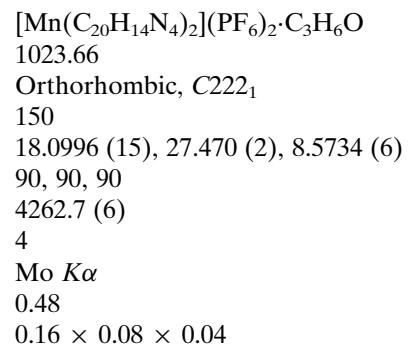

Bruker APEXII CCD

Multi-scan (SADABS; Bruker, 2007)

$0.953,0.960$

$44958,7445,5275$

Bruker APEXII CCD

Multi-scan (SADABS; Bruker, 2007)

$0.955,0.981$

$18921,3908,2683$

0.085

0.602

0.091

0.602

$0.089,0.244,1.07$

7445

608

78

$\mathrm{H}$-atom parameters constrained

$w=1 /\left[\sigma^{2}\left(F_{\mathrm{o}}^{2}\right)+(0.1065 P)^{2}+15.4857 P\right]$

where $P=\left(F_{\mathrm{o}}^{2}+2 F_{\mathrm{c}}^{2}\right) / 3$

$0.98,-1.03$

$0.104,0.270,1.07$

3908

292

102

$\mathrm{H}$-atom parameters constrained

$w=1 /\left[\sigma^{2}\left(F_{\mathrm{o}}{ }^{2}\right)+(0.0919 P)^{2}+48.9339 P\right]$

where $P=\left(F_{\mathrm{o}}^{2}+2 F_{\mathrm{c}}{ }^{2}\right) / 3$

$0.71,-1.05$

Flack $x$ determined using 836 quotients

$\left[\left(I^{+}\right)-\left(I^{-}\right)\right] /\left[\left(I^{+}\right)+\left(I^{-}\right)\right]$(Parsons et al. (2013) $0.21(2)$
Constable, Housecroft, Kepert, Price et al., 2007). Among the oligomers we can find linear structures with three (Liu et al., 2014) or five metal coordination centres (Beves et al., 2009) and hexanuclear cyclic clusters (Liu et al., 2014; see Table 8 for details).

\section{Synthesis and crystallization}

All the reactants were purchased from commercial suppliers and used as received.

\subsection{4'-(Pyridin-4-yl)-2, 2': $6^{\prime}, 2^{\prime \prime}$-terpyridine}

The ligand Pyterpy was synthesized by a mechanochemical reaction of 2-acetylpyridine, 4-pyridinecarboxaldehyde and $\mathrm{NaOH}$, followed by refluxing with ammonium acetate in acetic acid for $24 \mathrm{~h}$ (Cave \& Raston, 2001).

\subsection{Title compounds}

A solution of Pyterpy (132.7 mg, $0.42 \mathrm{mmol}$ ) in $\mathrm{MeOH}$ $(50 \mathrm{~mL})$ was added dropwise to a solution of $\left[\mathrm{Mn}\left(\mathrm{CH}_{3} \mathrm{COO}\right)_{2}\right] \cdot 4 \mathrm{H}_{2} \mathrm{O} \quad(52.4 \mathrm{mg}, 0.21 \mathrm{mmol})$ in $5 \mathrm{~mL}$ of water. The mixture refluxed at $338 \mathrm{~K}$ overnight to obtain a complete reaction. After this period, the solution was concentrated until a light-brown solid was obtained. The solid was filtrated and washed with water and ethanol to remove the impurities. The solid was dried at $333 \mathrm{~K}$. Analysis calculated for $\left[\mathrm{C}_{40} \mathrm{H}_{28} \mathrm{~F}_{12} \mathrm{MnN}_{8}\right]\left[\mathrm{PF}_{6}\right]_{2} \cdot \mathrm{H}_{2} \mathrm{O}: \mathrm{C} 47.97, \mathrm{H} 3.22, \mathrm{~N} 11.19 \%$; found: C 47.37, $\mathrm{H} 3.19, \mathrm{~N} 11.09 \%$.

Suitable crystals for X-ray diffraction were obtained by diffusion of water into a solution of the title compound in acetone. Two types of crystals were harvested corresponding to two different solvates.

\section{Refinement}

Crystal data, data collection and structure refinement details are summarized in Table 9.

$\mathrm{H}$ atoms bound to carbon were placed at their idealized positions and were included in the final structural model in riding-motion approximation, with $\mathrm{C}-\mathrm{H}=0.95 \AA$ (aromatic $\mathrm{C}-\mathrm{H}$ ) or $\mathrm{C}-\mathrm{H}=0.98 \AA$ (aliphatic $\mathrm{C}-\mathrm{H}$ ). The isotropic displacement parameters for these atoms were fixed at 1.2 times $U_{\text {eq }}$ of the respective parent carbon atom. Some parts of the two crystal structures are subjected to spatial disorder.

In (1), the disorder affects one whole ligand which is placed over two close, but not coincident locations, with occupancies 0.85:0.15. The two crystallographically independent $\mathrm{PF}_{6}{ }^{-}$ anions are distributed over four distinct orientations with 
coincidence of the central $\mathrm{P}$ atoms, and occupancies 2/3:1/3, $2 / 3: 1 / 3$. The $P$ atoms were refined anisotropically, and the $F$ atoms isotropically with a common $U_{\text {iso. }}$. The water molecule of crystallization was distributed over four distinct locations, which were isotropically refined with a common $U_{\text {iso, }}$ and total occupancy equal to 1 . The $\mathrm{H}$ atoms of the solvent were not located, but were added in the formula unit.

In (2), the disorder in the organic ligand was limited to a terminal 4-pyridine moiety, which was refined anisotropically over two locations with equal occupancies. The sole $\mathrm{PF}_{6}{ }^{-}$in (2) was distributed among two locations with occupancies 0.6:0.4 with P-atoms not coincident in space. The overall quality of the crystal was not sufficient for a precise determination of the Flack parameter.

\section{Acknowledgements}

We are grateful to Fundação para a Ciência e a Tecnologia (FCT Portugal) for general financial support to CICECO (PEst-C/CTM/LA0011/2013) and the R\&D project, including grant for LMOL, EXPL/QEQ-QUI/0199/2013 (FCOMP-010124-FEDER-041445), for postdoctoral research grant No. SFRH/BPD/63736/2009 (to JAF) and for specific funding toward the purchase of the single-crystal diffractometer.

\section{References}

Beves, J. E., Bray, D. J., Clegg, J. K., Constable, E. C., Housecroft, C. E., Jolliffe, K. A., Kepert, C. J., Lindoy, L. F., Neuburger, M., Price, D. J., Schaffner, S. \& Schaper, F. (2008). Inorg. Chim. Acta, 361, 2582-2590.

Beves, J. E., Constable, E. C., Decurtins, S., Dunphy, E. L., Housecroft, C. E., Keene, T. D., Neuburger, M., Schaffner, S. \& Zampese, J. A. (2009). CrystEngComm, 11, 2406-2416.

Beves, J. E., Constable, E. C., Housecroft, C. E., Kepert, C. J., Neuburger, M., Price, D. J. \& Schaffner, S. (2007). CrystEngComm, 9, 1073-1077.

Beves, J. E., Constable, E. C., Housecroft, C. E., Kepert, C. J. \& Price, D. J. (2007). CrystEngComm, 9, 456-459.
Beves, J. E., Constable, E. C., Housecroft, C. E., Neuburger, M. \& Schaffner, S. (2008). CrystEngComm, 10, 344-348.

Beves, J. E., Dunphy, E. L., Constable, E. C., Housecroft, C. E., Kepert, C. J., Neuburger, M., Price, D. J. \& Schaffner, S. (2008). Dalton Trans. pp. 386-396.

Bruker (2007). APEX2, SAINT and SADABS. Bruker AXS Inc., Madison, Wisconsin, USA.

Cave, G. W. V. \& Raston, C. L. (2001). J. Chem. Soc. Perkin Trans. 1, pp. 3258-3264.

Constable, E. C., Housecroft, C. E., Neuburger, M., Phillips, D., Raithby, P. R., Schofield, E., Sparr, E., Tocher, D. A., Zehnder, M. \& Zimmermann, Y. (2000). Dalton Trans. pp. 2219-2228.

Constable, E. C., Housecroft, C. E., Neuburger, M., Schaffner, S. \& Schaper, F. (2006). Inorg. Chem. Commun. 9, 616-619.

Ding, Y., Wang, F., Ku, Z.-J., Wang, L.-S. \& Zhou, H.-B. (2009). J. Struct. Chem. 50, 1212-1215.

Groom, C. R. \& Allen, F. H. (2014). Angew. Chem. Int. Ed. 53, 662671.

Halper, S. R., Do, L., Stork, J. R. \& Cohen, S. M. (2006). J. Am. Chem. Soc. 128, 15255-15268.

Indumathy, R., Radhika, S., Kanthimathi, M., Weyhermuller, T. \& Unni Nair, B. (2007). J. Inorg. Biochem. 101, 434-443.

Kitagawa, S., Noro, S. \& Nakamura, T. (2006). Chem. Commun. pp. 701-707.

Liu, J.-J., Lin, Y.-J. \& Jin, G.-X. (2014). Organometallics, 33, $1283-$ 1290.

Mehrani, A., Morsali, A. \& Ebrahimpour, P. (2013). J. Coord. Chem. 66, 856-867.

Morsali, A., Monfared, H. H., Ramazani, A., Noshiranzadeh, N., Morsali, A. \& Zeller, M. (2009). J. Coord. Chem. 62, 2631-2640.

Noro, S.-I., Miyasaka, H., Kitagawa, S., Wada, T., Okubo, T., Yamashita, M. \& Mitani, T. (2005). Inorg. Chem. Commun. 44, 133-146.

Parsons, S., Flack, H. D. \& Wagner, T. (2013). Acta Cryst. B69, 249 259.

Paul, J., Spey, S., Adams, H. \& Thomas, J. A. (2004). Inorg. Chim. Acta, 357, 2827-2832.

Pitarch López, J., Kraus, W., Reck, G., Thünemann, A. \& Kurth, D. G. (2005). Inorg. Chem. Commun. 8, 281-284.

Sheldrick, G. M. (2008). Acta Cryst. A64, 112-122.

Sheldrick, G. M. (2015). Acta Cryst. C71, 3-8.

Yoshida, J., Nishikiori, S.-I. \& Kuroda, R. (2009). Bull. Chem. Soc. Jpn, 82, 1377-1385. 


\section{supporting information}

Acta Cryst. (2015). E71, 330-335 [doi:10.1107/S2056989015003503]

Crystal structures of the water and acetone monosolvates of bis[4'-(pyridin-4yl)-2,2':6',2"'-terpyridine]manganese(II) bis(hexafluoridophosphate)

\section{Leandro M. O. Lourenço, Filipe A. Almeida Paz and José A. Fernandes}

Computing details

For both compounds, data collection: APEX2 (Bruker, 2007); cell refinement: SAINT (Bruker, 2007); data reduction: SAINT (Bruker, 2007); program(s) used to solve structure: SHELXS97 (Sheldrick, 2008). Program(s) used to refine structure: SHELXL2014 (Sheldrick, 2015) for (1); SHELXL97 (Sheldrick, 2008) for (2). For both compounds, molecular graphics: SHELXTL (Sheldrick, 2008); software used to prepare material for publication: SHELXTL (Sheldrick, 2008).

(1) Bis[4'-(pyridin-4-yl)-2,2':6',2"'-terpyridine]manganese(II) bis(hexafluoridophosphate) monohydrate

\section{Crystal data}

$\left[\mathrm{Mn}\left(\mathrm{C}_{20} \mathrm{H}_{14} \mathrm{~N}_{4}\right)_{2}\right]\left(\mathrm{PF}_{6}\right)_{2} \cdot \mathrm{H}_{2} \mathrm{O}$

$M_{r}=983.60$

Monoclinic, $P 2{ }_{1} / c$

$a=16.2389(5) \AA$

$b=15.3506(5) \AA$

$c=16.5549(5) \AA$

$\beta=99.3892(17)^{\circ}$

$V=4071.5(2) \AA^{3}$

$Z=4$

\section{Data collection}

Bruker APEXII CCD diffractometer

$\omega$ and $\varphi$ scans

Absorption correction: multi-scan

(SADABS; Bruker, 2007)

$T_{\min }=0.953, T_{\max }=0.960$

44958 measured reflections

\section{Refinement}

Refinement on $F^{2}$

Least-squares matrix: full

$R\left[F^{2}>2 \sigma\left(F^{2}\right)\right]=0.089$

$w R\left(F^{2}\right)=0.244$

$S=1.07$

7445 reflections

608 parameters

78 restraints
$F(000)=1988$

$D_{\mathrm{x}}=1.605 \mathrm{Mg} \mathrm{m}^{-3}$

Mo $K \alpha$ radiation, $\lambda=0.71073 \AA$

Cell parameters from 4312 reflections

$\theta=2.3-21.7^{\circ}$

$\mu=0.50 \mathrm{~mm}^{-1}$

$T=150 \mathrm{~K}$

Block, yellow

$0.12 \times 0.08 \times 0.08 \mathrm{~mm}$

7445 independent reflections

5275 reflections with $I>2 \sigma(I)$

$R_{\text {int }}=0.085$

$\theta_{\max }=25.4^{\circ}, \theta_{\min }=2.7^{\circ}$

$h=-19 \rightarrow 19$

$k=-18 \rightarrow 17$

$l=-19 \rightarrow 17$

Hydrogen site location: inferred from neighbouring sites

$\mathrm{H}$-atom parameters constrained

$w=1 /\left[\sigma^{2}\left(F_{\mathrm{o}}{ }^{2}\right)+(0.1065 P)^{2}+15.4857 P\right]$

where $P=\left(F_{\mathrm{o}}^{2}+2 F_{\mathrm{c}}^{2}\right) / 3$

$(\Delta / \sigma)_{\max }<0.001$

$\Delta \rho_{\max }=0.98$ e $\AA^{-3}$

$\Delta \rho_{\min }=-1.03 \mathrm{e} \AA^{-3}$ 


\section{Special details}

Geometry. All e.s.d.'s (except the e.s.d. in the dihedral angle between two 1.s. planes) are estimated using the full covariance matrix. The cell e.s.d.'s are taken into account individually in the estimation of e.s.d.'s in distances, angles and torsion angles; correlations between e.s.d.'s in cell parameters are only used when they are defined by crystal symmetry. An approximate (isotropic) treatment of cell e.s.d.'s is used for estimating e.s.d.'s involving l.s. planes.

Fractional atomic coordinates and isotropic or equivalent isotropic displacement parameters $\left(\hat{A}^{2}\right)$

\begin{tabular}{|c|c|c|c|c|c|}
\hline & $x$ & $y$ & $z$ & $U_{\text {iso }} * / U_{\text {eq }}$ & Occ. $(<1)$ \\
\hline Mn1 & $0.23762(5)$ & $0.32425(5)$ & $0.12734(5)$ & $0.0275(2)$ & \\
\hline $\mathrm{C} 1$ & 0.2558 & $0.3471(4)$ & $0.3199(3)$ & $0.0346(13)$ & \\
\hline $\mathrm{H} 1$ & 0.1969 & 0.3397 & 0.3092 & $0.042^{*}$ & \\
\hline $\mathrm{C} 2$ & $0.2943(4)$ & $0.3585(4)$ & $0.3993(3)$ & $0.0416(15)$ & \\
\hline $\mathrm{H} 2$ & 0.2625 & 0.3604 & 0.4426 & $0.050^{*}$ & \\
\hline $\mathrm{C} 3$ & $0.3804(4)$ & $0.3672(4)$ & $0.4151(3)$ & $0.0427(15)$ & \\
\hline H3 & 0.4087 & 0.3741 & 0.4697 & $0.051^{*}$ & \\
\hline $\mathrm{C} 4$ & $0.4244(3)$ & $0.3657(4)$ & $0.3509(3)$ & $0.0370(13)$ & \\
\hline $\mathrm{H} 4$ & 0.4835 & 0.3715 & 0.3608 & $0.044 *$ & \\
\hline $\mathrm{C} 5$ & $0.3820(3)$ & $0.3557(3)$ & $0.2717(3)$ & $0.0249(11)$ & \\
\hline C6 & $0.4226(3)$ & $0.3574(3)$ & $0.1976(3)$ & $0.0238(10)$ & \\
\hline $\mathrm{C} 7$ & $0.5077(3)$ & $0.3676(3)$ & $0.1992(3)$ & $0.0290(11)$ & \\
\hline $\mathrm{H} 7$ & 0.5443 & 0.3718 & 0.2501 & $0.035^{*}$ & \\
\hline $\mathrm{C} 8$ & 0.5394 & $0.3716(3)$ & $0.1263(3)$ & $0.0283(11)$ & \\
\hline C9 & $0.4837(3)$ & $0.3648(3)$ & $0.0532(3)$ & $0.0281(11)$ & \\
\hline H9 & 0.5033 & 0.3662 & 0.0022 & $0.034^{*}$ & \\
\hline $\mathrm{C} 10$ & $0.3995(3)$ & 0.3559 (3) & $0.0556(3)$ & $0.0259(11)$ & \\
\hline $\mathrm{C} 11$ & $0.3337(3)$ & $0.3549(4)$ & $-0.0184(3)$ & $0.0300(12)$ & \\
\hline $\mathrm{C} 12$ & $0.3511(3)$ & $0.3682(4)$ & $-0.0969(3)$ & $0.0376(14)$ & \\
\hline H12 & 0.4070 & 0.3751 & -0.1059 & $0.045^{*}$ & \\
\hline $\mathrm{C} 13$ & $0.2859(4)$ & $0.3713(4)$ & $-0.1616(4)$ & $0.0439(15)$ & \\
\hline H13 & 0.2965 & 0.3822 & -0.2154 & $0.053^{*}$ & \\
\hline C14 & $0.2048(4)$ & $0.3586(4)$ & $-0.1479(3)$ & 0.0408 (14) & \\
\hline H14 & 0.1592 & 0.3599 & -0.1918 & $0.049^{*}$ & \\
\hline $\mathrm{C} 15$ & $0.1922(3)$ & $0.3440(4)$ & $-0.0678(3)$ & $0.0359(13)$ & \\
\hline H15 & 0.1370 & 0.3346 & -0.0577 & $0.043^{*}$ & \\
\hline $\mathrm{C} 16$ & $0.7731(4)$ & $0.3864(5)$ & 0.1888 & $0.0526(18)$ & \\
\hline H16 & 0.8130 & 0.3756 & 0.2364 & $0.063^{*}$ & \\
\hline $\mathrm{C} 17$ & $0.6904(4)$ & $0.3686(5)$ & $0.1921(4)$ & $0.0511(18)$ & \\
\hline H17 & 0.6751 & 0.3452 & 0.2406 & $0.061^{*}$ & \\
\hline C18 & 0.6299 & $0.3849(4)$ & $0.1250(3)$ & $0.0313(12)$ & \\
\hline C19 & $0.6575(4)$ & $0.4209(5)$ & $0.0581(4)$ & $0.0488(16)$ & \\
\hline H19 & 0.6187 & 0.4355 & 0.0106 & $0.059^{*}$ & \\
\hline $\mathrm{C} 20$ & $0.7424(4)$ & $0.4359(5)$ & $0.0600(4)$ & 0.0577 (19) & \\
\hline $\mathrm{H} 20$ & 0.7598 & 0.4609 & 0.0131 & $0.069^{*}$ & \\
\hline N1 & 0.2981 & $0.3460(3)$ & $0.2567(3)$ & $0.0279(9)$ & \\
\hline $\mathrm{N} 2$ & 0.3699 & $0.3521(3)$ & $0.1267(3)$ & $0.0267(9)$ & \\
\hline N3 & 0.2550 & $0.3426(3)$ & $-0.0048(3)$ & $0.0290(10)$ & \\
\hline N4 & $0.8001(3)$ & $0.4173(4)$ & $0.1237(3)$ & $0.0484(14)$ & \\
\hline
\end{tabular}




\begin{tabular}{|c|c|c|c|c|c|}
\hline $\mathrm{C} 21$ & $0.3152(3)$ & $0.1327(4)$ & $0.1204(8)$ & $0.0301(13)$ & 0.85 \\
\hline $\mathrm{H} 21$ & 0.3661 & 0.1641 & 0.1243 & $0.036^{*}$ & 0.85 \\
\hline $\mathrm{C} 22$ & $0.3177(4)$ & 0.0437 (4) & $0.1137(8)$ & $0.0398(16)$ & 0.85 \\
\hline $\mathrm{H} 22$ & 0.3689 & 0.0141 & 0.1125 & $0.048^{*}$ & 0.85 \\
\hline $\mathrm{C} 23$ & $0.2438(4)$ & $-0.0015(5)$ & $0.1086(8)$ & $0.0507(19)$ & 0.85 \\
\hline $\mathrm{H} 23$ & 0.2433 & -0.0632 & 0.1038 & $0.061 *$ & 0.85 \\
\hline $\mathrm{C} 24$ & $0.1706(4)$ & 0.0433 & $0.1104(8)$ & $0.0450(17)$ & 0.85 \\
\hline $\mathrm{H} 24$ & 0.1194 & 0.0126 & 0.1073 & $0.054^{*}$ & 0.85 \\
\hline $\mathrm{C} 25$ & $0.1721(3)$ & $0.1329(4)$ & $0.1167(6)$ & $0.0295(13)$ & 0.85 \\
\hline $\mathrm{C} 26$ & $0.0960(3)$ & $0.1864(4)$ & $0.1156(6)$ & $0.0311(14)$ & 0.85 \\
\hline $\mathrm{C} 27$ & $0.0159(4)$ & 0.1533 & 0.1074 (4) & $0.0333(17)$ & 0.85 \\
\hline $\mathrm{H} 27$ & 0.0070 & 0.0921 & 0.1069 & $0.040^{*}$ & 0.85 \\
\hline $\mathrm{C} 29$ & $-0.0365(3)$ & $0.2992(5)$ & 0.1051 (4) & $0.0332(18)$ & 0.85 \\
\hline $\mathrm{H} 29$ & -0.0815 & 0.3393 & 0.1004 & $0.040^{*}$ & 0.85 \\
\hline $\mathrm{C} 30$ & $0.0456(3)$ & $0.3287(4)$ & $0.1172(6)$ & $0.0295(14)$ & 0.85 \\
\hline $\mathrm{C} 31$ & $0.0684(3)$ & 0.4218 & $0.1253(7)$ & $0.0288(13)$ & 0.85 \\
\hline C32 & $0.0107(4)$ & 0.4877 (4) & $0.1306(8)$ & $0.037(2)$ & 0.85 \\
\hline H32 & -0.0469 & 0.4745 & 0.1274 & $0.045^{*}$ & 0.85 \\
\hline $\mathrm{C} 33$ & 0.0385 (4) & $0.5721(5)$ & 0.1407 (4) & $0.039(2)$ & 0.85 \\
\hline $\mathrm{H} 33$ & 0.0001 & 0.6181 & 0.1442 & $0.047^{*}$ & 0.85 \\
\hline $\mathrm{C} 34$ & $0.1223(4)$ & $0.5897(4)$ & $0.1456(10)$ & $0.039(2)$ & 0.85 \\
\hline H34 & 0.1424 & 0.6478 & 0.1524 & $0.047^{*}$ & 0.85 \\
\hline $\mathrm{C} 35$ & $0.1759(4)$ & 0.5220 & $0.1405(15)$ & $0.0365(15)$ & 0.85 \\
\hline H35 & 0.2337 & 0.5344 & 0.1440 & $0.044^{*}$ & 0.85 \\
\hline N5 & $0.2449(3)$ & $0.1775(3)$ & $0.1218(7)$ & $0.0268(10)$ & 0.85 \\
\hline N6 & 0.1094 (3) & $0.2730(3)$ & $0.1208(6)$ & $0.0267(10)$ & 0.85 \\
\hline N7 & $0.1508(3)$ & $0.4387(3)$ & $0.1308(9)$ & $0.0282(16)$ & 0.85 \\
\hline $\mathrm{C} 28$ & $-0.0515(4)$ & $0.2103(5)$ & 0.0998 (4) & $0.0295(15)$ & 0.85 \\
\hline C36 & $-0.2505(6)$ & $0.0891(6)$ & 0.1153 (6) & $0.055(2)$ & 0.85 \\
\hline H36 & -0.2682 & 0.0424 & 0.1460 & $0.066^{*}$ & 0.85 \\
\hline $\mathrm{C} 37$ & $-0.1654(4)$ & $0.1106(5)$ & $0.1289(5)$ & $0.0437(17)$ & 0.85 \\
\hline H37 & -0.1266 & 0.0786 & 0.1667 & $0.052^{*}$ & 0.85 \\
\hline C38 & -0.1400 & $0.1792(5)$ & 0.0859 (4) & $0.0362(15)$ & 0.85 \\
\hline C39 & -0.1991 & $0.2225(5)$ & $0.0295(5)$ & $0.0427(17)$ & 0.85 \\
\hline H39 & -0.1833 & 0.2700 & -0.0015 & $0.051^{*}$ & 0.85 \\
\hline $\mathrm{C} 40$ & $-0.2812(4)$ & $0.1948(5)$ & $0.0196(5)$ & $0.0455(18)$ & 0.85 \\
\hline $\mathrm{H} 40$ & -0.3209 & 0.2237 & -0.0200 & $0.055^{*}$ & 0.85 \\
\hline N8 & -0.3080 & $0.1298(4)$ & $0.0623(4)$ & $0.0504(16)$ & 0.85 \\
\hline $\mathrm{C} 121$ & $0.3340(10)$ & $0.1304(9)$ & $0.125(5)$ & $0.0301(13)$ & 0.15 \\
\hline H121 & 0.3835 & 0.1642 & 0.1291 & $0.036^{*}$ & 0.15 \\
\hline C122 & $0.3405(11)$ & $0.0416(10)$ & $0.119(5)$ & $0.0398(16)$ & 0.15 \\
\hline H122 & 0.3932 & 0.0143 & 0.1208 & $0.048^{*}$ & 0.15 \\
\hline $\mathrm{C} 123$ & $0.2683(11)$ & $-0.0068(9)$ & $0.112(5)$ & 0.0507 (19) & 0.15 \\
\hline H123 & 0.2702 & -0.0682 & 0.1053 & $0.061^{*}$ & 0.15 \\
\hline C124 & $0.1933(11)$ & $0.0343(8)$ & $0.114(5)$ & $0.0450(17)$ & 0.15 \\
\hline H124 & 0.1435 & 0.0012 & 0.1113 & $0.054 *$ & 0.15 \\
\hline $\mathrm{C} 125$ & $0.1911(8)$ & $0.1238(7)$ & $0.122(3)$ & $0.0295(13)$ & 0.15 \\
\hline $\mathrm{C} 126$ & $0.1131(8)$ & $0.1734(6)$ & $0.123(3)$ & $0.0311(14)$ & 0.15 \\
\hline
\end{tabular}




\begin{tabular}{|c|c|c|c|c|c|}
\hline C127 & $0.0362(10)$ & $0.1360(7)$ & $0.126(3)$ & $0.0333(17)$ & 0.15 \\
\hline H127 & 0.0326 & 0.0743 & 0.1275 & $0.040^{*}$ & 0.15 \\
\hline C129 & $-0.0227(9)$ & $0.2790(9)$ & $0.126(3)$ & $0.0332(18)$ & 0.15 \\
\hline H129 & -0.0685 & 0.3174 & 0.1266 & $0.040^{*}$ & 0.15 \\
\hline C130 & $0.0565(7)$ & $0.3130(7)$ & $0.125(3)$ & $0.0295(14)$ & 0.15 \\
\hline C131 & $0.0750(7)$ & $0.4073(7)$ & $0.130(4)$ & $0.0288(13)$ & 0.15 \\
\hline C132 & $0.0134(8)$ & $0.4711(7)$ & $0.119(5)$ & $0.037(2)$ & 0.15 \\
\hline H132 & -0.0439 & 0.4556 & 0.1131 & $0.045^{*}$ & 0.15 \\
\hline C133 & $0.0370(10)$ & $0.5570(8)$ & $0.119(3)$ & 0.039 (2) & 0.15 \\
\hline H133 & -0.0041 & 0.6015 & 0.1084 & $0.047^{*}$ & 0.15 \\
\hline $\mathrm{C} 134$ & $0.1204(10)$ & $0.5783(7)$ & $0.133(7)$ & $0.039(2)$ & 0.15 \\
\hline H134 & 0.1376 & 0.6375 & 0.1362 & $0.047^{*}$ & 0.15 \\
\hline C135 & $0.1779(9)$ & $0.5125(8)$ & $0.143(9)$ & $0.0365(15)$ & 0.15 \\
\hline H135 & 0.2355 & 0.5274 & 0.1528 & $0.044^{*}$ & 0.15 \\
\hline N105 & $0.2616(8)$ & $0.1719(7)$ & $0.125(4)$ & $0.0268(10)$ & 0.15 \\
\hline N106 & $0.1221(7)$ & $0.2607(6)$ & $0.124(3)$ & $0.0267(10)$ & 0.15 \\
\hline N107 & $0.1569(7)$ & $0.4278(7)$ & $0.139(6)$ & $0.0282(16)$ & 0.15 \\
\hline $\mathrm{C} 128$ & $-0.036(3)$ & 0.185 & $0.127(3)$ & $0.029(3)^{*}$ & 0.15 \\
\hline C136 & $-0.2565(12)$ & $0.1536(15)$ & $0.1642(14)$ & $0.029(3)^{*}$ & 0.15 \\
\hline H136 & -0.2954 & 0.1803 & 0.1935 & $0.035^{*}$ & 0.15 \\
\hline $\mathrm{C} 137$ & $-0.1765(13)$ & $0.1876(13)$ & $0.1695(14)$ & $0.029(3)^{*}$ & 0.15 \\
\hline H137 & -0.1607 & 0.2374 & 0.2023 & $0.035^{*}$ & 0.15 \\
\hline C138 & $-0.1195(10)$ & $0.1486(15)$ & $0.1266(15)$ & $0.029(3)^{*}$ & 0.15 \\
\hline C139 & $-0.1425(12)$ & $0.0756(14)$ & $0.0785(14)$ & $0.029(3)^{*}$ & 0.15 \\
\hline H139 & -0.1036 & 0.0490 & 0.0492 & $0.035^{*}$ & 0.15 \\
\hline C140 & $-0.2226(13)$ & $0.0417(13)$ & $0.0732(14)$ & $0.029(3)^{*}$ & 0.15 \\
\hline H140 & -0.2384 & -0.0082 & 0.0403 & $0.035^{*}$ & 0.15 \\
\hline N108 & $-0.2796(11)$ & $0.0807(16)$ & $0.1160(16)$ & $0.039(13)^{*}$ & 0.15 \\
\hline P1A & $0.42327(11)$ & $0.62842(12)$ & $0.19001(12)$ & $0.0535(5)$ & 0.6667 \\
\hline F1A & $0.3907(5)$ & $0.5541(6)$ & $0.2411(5)$ & $0.0670(5)^{*}$ & 0.6667 \\
\hline F2A & $0.3333(4)$ & $0.6356(5)$ & $0.1268(4)$ & $0.0670(5)^{*}$ & 0.6667 \\
\hline F3A & $0.4501(4)$ & $0.7046(4)$ & $0.1273(4)$ & $0.0670(5)^{*}$ & 0.6667 \\
\hline $\mathrm{F} 4 \mathrm{~A}$ & $0.5117(4)$ & $0.6332(5)$ & $0.2411(4)$ & $0.0670(5)^{*}$ & 0.6667 \\
\hline F5A & $0.4558(4)$ & $0.5636(5)$ & $0.1279(5)$ & $0.0670(5)^{*}$ & 0.6667 \\
\hline F6A & $0.3903(4)$ & $0.7023(5)$ & $0.2432(4)$ & $0.0670(5)^{*}$ & 0.6667 \\
\hline P1B & $0.42327(11)$ & $0.62842(12)$ & $0.19001(12)$ & $0.0535(5)$ & 0.3333 \\
\hline F1B & $0.3743(9)$ & $0.5529(11)$ & $0.2303(10)$ & $0.0670(5)^{*}$ & 0.3333 \\
\hline F2B & $0.3353(7)$ & $0.6633(9)$ & $0.1540(8)$ & $0.0670(5)^{*}$ & 0.3333 \\
\hline F3B & $0.4753(8)$ & $0.7007(8)$ & $0.1577(8)$ & $0.0670(5)^{*}$ & 0.3333 \\
\hline F4B & $0.5110(7)$ & $0.5810(9)$ & $0.2381(8)$ & $0.0670(5)^{*}$ & 0.3333 \\
\hline F5B & $0.4312(8)$ & $0.5591(9)$ & $0.1211(9)$ & $0.0670(5)^{*}$ & 0.3333 \\
\hline F6B & $0.4290(9)$ & $0.6817(9)$ & $0.2777(9)$ & $0.0670(5)^{*}$ & 0.3333 \\
\hline $\mathrm{P} 2 \mathrm{~A}$ & $0.01085(12)$ & $0.34258(13)$ & $0.37053(11)$ & $0.0515(5)$ & 0.6667 \\
\hline F7A & $-0.0799(4)$ & $0.3052(5)$ & 0.3288 & $0.0670(5)^{*}$ & 0.6667 \\
\hline F8A & $0.0523(6)$ & $0.2825(6)$ & $0.3097(6)$ & $0.0670(5)^{*}$ & 0.6667 \\
\hline F9A & $0.1001(4)$ & $0.3682(4)$ & $0.4138(4)$ & $0.0670(5)^{*}$ & 0.6667 \\
\hline F10A & $-0.0116(4)$ & $0.4151(5)$ & $0.4360(4)$ & $0.0670(5)^{*}$ & 0.6667 \\
\hline F11A & -0.0065 & $0.4169(4)$ & $0.3052(4)$ & $0.0670(5)^{*}$ & 0.6667 \\
\hline
\end{tabular}




\begin{tabular}{llllll} 
F12A & $0.0152(4)$ & $0.2618(5)$ & $0.4337(5)$ & $0.0670(5)^{*}$ & 0.6667 \\
P2B & $0.01085(12)$ & $0.34258(13)$ & $0.37053(11)$ & $0.0515(5)$ & 0.3333 \\
F7B & $-0.0657(7)$ & $0.3666(9)$ & $0.3129(7)$ & $0.0670(5)^{*}$ & 0.3333 \\
F8B & $0.0417(11)$ & $0.2716(10)$ & $0.3099(11)$ & $0.0670(5)^{*}$ & 0.3333 \\
F9B & $0.1034(7)$ & $0.3303(9)$ & $0.4304(8)$ & $0.0670(5)^{*}$ & 0.3333 \\
F10B & $-0.0470(9)$ & $0.3850(9)$ & $0.4251(9)$ & $0.0670(5)^{*}$ & 0.3333 \\
F11B & $0.0566(8)$ & $0.4202(8)$ & $0.3231(8)$ & $0.0670(5)^{*}$ & 0.3333 \\
F12B & $-0.0136(8)$ & $0.2662(9)$ & $0.4254(9)$ & $0.0670(5)^{*}$ & 0.3333 \\
O1W & $0.5185(7)$ & $0.3890(7)$ & $0.5830(7)$ & $0.0411(19)^{*}$ & 0.4 \\
O2W & $0.5228(8)$ & $0.4277(8)$ & $0.5626(8)$ & $0.0411(19)^{*}$ & 0.35 \\
O3W & $0.5531(17)$ & $0.4242(18)$ & $0.5175(17)$ & $0.0411(19)^{*}$ & 0.15 \\
O4W & $0.637(3)$ & $0.350(3)$ & $0.392(3)$ & $0.0411(19)^{*}$ & 0.1 \\
\hline
\end{tabular}

Atomic displacement parameters $\left(\AA^{2}\right)$

\begin{tabular}{|c|c|c|c|c|c|c|}
\hline & $U^{11}$ & $U^{22}$ & $U^{33}$ & $U^{12}$ & $U^{13}$ & $U^{23}$ \\
\hline Mn1 & 0.0196 & $0.0389(5)$ & 0.0244 & -0.0075 & $0.0051(3)$ & $-0.0017(3)$ \\
\hline $\mathrm{C} 1$ & $0.030(3)$ & $0.046(3)$ & $0.029(3)$ & $-0.003(2)$ & 0.009 (2) & $0.000(2)$ \\
\hline $\mathrm{C} 2$ & $0.036(3)$ & $0.072(4)$ & 0.018 & $0.001(3)$ & 0.009 (2) & 0.000 \\
\hline $\mathrm{C} 3$ & 0.037 (3) & 0.070 (4) & $0.020(3)$ & $0.003(3)$ & $0.003(2)$ & -0.007 (3) \\
\hline $\mathrm{C} 4$ & $0.025(3)$ & $0.057(4)$ & $0.028(3)$ & -0.002 & $0.002(2)$ & $0.002(3)$ \\
\hline $\mathrm{C} 5$ & $0.022(2)$ & $0.027(3)$ & $0.025(3)$ & $-0.001(2)$ & $0.002(2)$ & $0.000(2)$ \\
\hline C6 & $0.022(2)$ & $0.028(3)$ & $0.022(2)$ & $-0.002(2)$ & $0.004(2)$ & $0.000(2)$ \\
\hline $\mathrm{C} 7$ & $0.022(2)$ & $0.038(3)$ & $0.026(3)$ & $0.000(2)$ & $0.000(2)$ & $0.002(2)$ \\
\hline C8 & $0.020(2)$ & $0.033(3)$ & $0.032(3)$ & $0.002(2)$ & $0.004(2)$ & $0.001(2)$ \\
\hline C9 & $0.025(3)$ & $0.035(3)$ & $0.026(3)$ & $-0.006(2)$ & $0.008(2)$ & $0.001(2)$ \\
\hline $\mathrm{C} 10$ & $0.023(2)$ & $0.031(3)$ & $0.023(3)$ & $-0.002(2)$ & $0.004(2)$ & $-0.003(2)$ \\
\hline $\mathrm{C} 11$ & $0.023(3)$ & $0.044(3)$ & $0.022(3)$ & $-0.006(2)$ & $0.002(2)$ & $-0.002(2)$ \\
\hline $\mathrm{C} 12$ & $0.026(3)$ & $0.060(4)$ & $0.027(3)$ & $-0.012(3)$ & $0.008(2)$ & $-0.004(3)$ \\
\hline $\mathrm{C} 13$ & $0.040(3)$ & $0.066(4)$ & $0.026(3)$ & -0.008 & $0.006(3)$ & $-0.002(3)$ \\
\hline $\mathrm{C} 14$ & $0.030(3)$ & $0.064(4)$ & $0.027(3)$ & -0.004 & $0.000(2)$ & $-0.002(3)$ \\
\hline $\mathrm{C} 15$ & $0.028(3)$ & $0.054(4)$ & $0.026(3)$ & $-0.012(3)$ & $0.004(2)$ & $0.002(3)$ \\
\hline $\mathrm{C} 16$ & $0.023(3)$ & $0.086(5)$ & $0.047(4)$ & 0.000 & $0.001(3)$ & 0.009 (4) \\
\hline $\mathrm{C} 17$ & $0.022(3)$ & $0.088(5)$ & $0.043(4)$ & -0.003 & $0.002(3)$ & $0.011(3)$ \\
\hline C18 & $0.020(3)$ & $0.036(3)$ & $0.039(3)$ & $0.004(2)$ & $0.007(2)$ & $0.003(2)$ \\
\hline C19 & $0.028(3)$ & $0.074(5)$ & $0.045(4)$ & $-0.002(3)$ & 0.008 & $0.004(3)$ \\
\hline $\mathrm{C} 20$ & $0.036(4)$ & $0.087(5)$ & $0.054(4)$ & $-0.014(4)$ & $0.020(3)$ & $-0.002(4)$ \\
\hline N1 & $0.024(2)$ & $0.033(2)$ & $0.027(2)$ & $-0.0025(18)$ & $0.0052(18)$ & $-0.0021(19)$ \\
\hline $\mathrm{N} 2$ & $0.021(2)$ & $0.034(2)$ & $0.026(2)$ & $-0.0023(18)$ & $0.0061(17)$ & $0.0006(18)$ \\
\hline N3 & $0.021(2)$ & $0.040(3)$ & $0.025(2)$ & $-0.0082(19)$ & $0.0018(17)$ & $-0.0023(19)$ \\
\hline $\mathrm{N} 4$ & $0.022(2)$ & $0.069(4)$ & $0.054(3)$ & $0.000(2)$ & $0.005(2)$ & 0.000 \\
\hline $\mathrm{C} 21$ & $0.023(3)$ & $0.042(3)$ & $0.024(3)$ & $0.000(3)$ & $-0.001(4)$ & $0.006(2)$ \\
\hline $\mathrm{C} 22$ & $0.033(4)$ & $0.044(4)$ & $0.041(4)$ & $0.005(3)$ & $0.002(5)$ & $0.006(3)$ \\
\hline $\mathrm{C} 23$ & $0.041(5)$ & $0.036(4)$ & $0.071(5)$ & -0.003 & $-0.002(5)$ & $0.004(3)$ \\
\hline $\mathrm{C} 24$ & $0.027(4)$ & $0.050(4)$ & $0.056(4)$ & $-0.007(3)$ & $0.002(5)$ & $0.002(4)$ \\
\hline $\mathrm{C} 25$ & $0.022(3)$ & $0.038(3)$ & $0.029(3)$ & $-0.010(2)$ & $0.002(3)$ & $0.000(3)$ \\
\hline $\mathrm{C} 26$ & $0.024(3)$ & $0.045(4)$ & $0.024(3)$ & $-0.012(3)$ & $0.004(3)$ & $-0.002(3)$ \\
\hline $\mathrm{C} 27$ & $0.026(3)$ & $0.043(4)$ & $0.032(5)$ & -0.009 (3) & $0.007(3)$ & -0.001 \\
\hline
\end{tabular}




\begin{tabular}{|c|c|c|c|c|c|c|}
\hline $\mathrm{C} 29$ & $0.019(3)$ & $0.048(4)$ & $0.034(5)$ & $-0.004(3)$ & $0.006(3)$ & $-0.008(3)$ \\
\hline $\mathrm{C} 30$ & $0.019(3)$ & $0.042(3)$ & $0.028(3)$ & $-0.006(2)$ & $0.007(3)$ & $-0.005(3)$ \\
\hline $\mathrm{C} 31$ & $0.018(2)$ & $0.045(3)$ & $0.023(3)$ & $-0.002(2)$ & $0.004(2)$ & $-0.002(3)$ \\
\hline $\mathrm{C} 32$ & $0.022(3)$ & 0.047 (4) & $0.043(5)$ & $-0.005(3)$ & $0.009(3)$ & $-0.012(4)$ \\
\hline $\mathrm{C} 33$ & $0.029(3)$ & $0.050(4)$ & $0.038(5)$ & $0.002(3)$ & $0.005(3)$ & $-0.012(3)$ \\
\hline $\mathrm{C} 34$ & $0.041(3)$ & $0.034(3)$ & $0.042(7)$ & $-0.009(3)$ & $0.005(3)$ & $-0.010(4)$ \\
\hline $\mathrm{C} 35$ & $0.027(3)$ & $0.045(4)$ & $0.040(4)$ & $-0.005(3)$ & $0.011(3)$ & $-0.002(4)$ \\
\hline N5 & $0.024(3)$ & $0.035(3)$ & $0.022(2)$ & -0.003 & $0.004(3)$ & $-0.001(2)$ \\
\hline N6 & $0.018(2)$ & $0.036(3)$ & $0.027(2)$ & $-0.005(2)$ & $0.006(2)$ & $-0.002(2)$ \\
\hline N7 & $0.018(2)$ & $0.039(3)$ & $0.028(5)$ & $-0.0029(19)$ & $0.005(2)$ & $-0.002(3)$ \\
\hline $\mathrm{C} 28$ & $0.023(3)$ & $0.038(4)$ & $0.028(4)$ & $-0.007(3)$ & 0.007 & $0.001(3)$ \\
\hline $\mathrm{C} 36$ & $0.040(5)$ & $0.064(6)$ & $0.061(6)$ & $-0.015(4)$ & $0.012(4)$ & $0.012(4)$ \\
\hline $\mathrm{C} 37$ & $0.025(3)$ & $0.052(4)$ & $0.054(4)$ & $-0.010(3)$ & $0.004(3)$ & $0.005(4)$ \\
\hline $\mathrm{C} 38$ & $0.024(3)$ & 0.047 (4) & $0.038(4)$ & $-0.010(3)$ & $0.006(3)$ & $-0.007(3)$ \\
\hline C39 & $0.031(4)$ & $0.052(4)$ & $0.045(4)$ & $-0.010(3)$ & $0.006(3)$ & $-0.006(3)$ \\
\hline $\mathrm{C} 40$ & $0.026(3)$ & $0.058(5)$ & $0.050(4)$ & -0.003 & $-0.001(3)$ & -0.014 (4) \\
\hline N8 & $0.027(3)$ & $0.057(4)$ & $0.067(4)$ & $-0.011(3)$ & $0.008(3)$ & $-0.004(4)$ \\
\hline $\mathrm{C} 121$ & $0.023(3)$ & $0.042(3)$ & $0.024(3)$ & $0.000(3)$ & $-0.001(4)$ & $0.006(2)$ \\
\hline $\mathrm{C} 122$ & $0.033(4)$ & $0.044(4)$ & $0.041(4)$ & $0.005(3)$ & $0.002(5)$ & $0.006(3)$ \\
\hline C123 & $0.041(5)$ & $0.036(4)$ & $0.071(5)$ & -0.003 & $-0.002(5)$ & $0.004(3)$ \\
\hline $\mathrm{C} 124$ & $0.027(4)$ & $0.050(4)$ & $0.056(4)$ & $-0.007(3)$ & $0.002(5)$ & $0.002(4)$ \\
\hline $\mathrm{C} 125$ & $0.022(3)$ & $0.038(3)$ & $0.029(3)$ & $-0.010(2)$ & $0.002(3)$ & $0.000(3)$ \\
\hline $\mathrm{C} 126$ & $0.024(3)$ & $0.045(4)$ & $0.024(3)$ & $-0.012(3)$ & $0.004(3)$ & $-0.002(3)$ \\
\hline $\mathrm{C} 127$ & $0.026(3)$ & $0.043(4)$ & $0.032(5)$ & $-0.009(3)$ & $0.007(3)$ & $-0.001(3)$ \\
\hline C129 & $0.019(3)$ & $0.048(4)$ & $0.034(5)$ & $-0.004(3)$ & $0.006(3)$ & $-0.008(3)$ \\
\hline $\mathrm{C} 130$ & $0.019(3)$ & $0.042(3)$ & $0.028(3)$ & $-0.006(2)$ & $0.007(3)$ & $-0.005(3)$ \\
\hline $\mathrm{C} 131$ & $0.018(2)$ & $0.045(3)$ & $0.023(3)$ & $-0.002(2)$ & $0.004(2)$ & $-0.002(3)$ \\
\hline $\mathrm{C} 132$ & $0.022(3)$ & $0.047(4)$ & $0.043(5)$ & $-0.005(3)$ & $0.009(3)$ & $-0.012(4)$ \\
\hline $\mathrm{C} 133$ & $0.029(3)$ & $0.050(4)$ & $0.038(5)$ & $0.002(3)$ & 0.005 & $-0.012(3)$ \\
\hline $\mathrm{C} 134$ & $0.041(3)$ & $0.034(3)$ & $0.042(7)$ & $-0.009(3)$ & 0.005 & $-0.010(4)$ \\
\hline $\mathrm{C} 135$ & $0.027(3)$ & $0.045(4)$ & $0.040(4)$ & $-0.005(3)$ & $0.011(3)$ & $-0.002(4)$ \\
\hline N105 & $0.024(3)$ & $0.035(3)$ & $0.022(2)$ & $-0.003(2)$ & $0.004(3)$ & $-0.001(2)$ \\
\hline N106 & $0.018(2)$ & $0.036(3)$ & $0.027(2)$ & $-0.005(2)$ & $0.006(2)$ & $-0.002(2)$ \\
\hline N107 & $0.018(2)$ & $0.039(3)$ & $0.028(5)$ & $-0.0029(19)$ & $0.005(2)$ & $-0.002(3)$ \\
\hline P1A & $0.0462(10)$ & $0.0529(11)$ & $0.0672(12)$ & $-0.0157(8)$ & $0.0263(9)$ & $-0.0193(9)$ \\
\hline P1B & $0.0462(10)$ & $0.0529(11)$ & $0.0672(12)$ & $-0.0157(8)$ & $0.0263(9)$ & $-0.0193(9)$ \\
\hline P2A & $0.0512(10)$ & $0.0674(12)$ & $0.0375(9)$ & $0.0224(9)$ & $0.0124(8)$ & $0.0119(8)$ \\
\hline P2B & $0.0512(10)$ & $0.0674(12)$ & $0.0375(9)$ & $0.0224(9)$ & $0.0124(8)$ & $0.0119(8)$ \\
\hline
\end{tabular}

Geometric parameters $\left(\AA,{ }^{\circ}\right)$

\begin{tabular}{llll}
\hline $\mathrm{Mn} 1-\mathrm{N} 1$ & $2.231(4)$ & $\mathrm{C} 28-\mathrm{C} 38$ & $1.496(9)$ \\
$\mathrm{Mn} 1-\mathrm{N} 2$ & $2.193(4)$ & $\mathrm{C} 36-\mathrm{N} 8$ & $1.328(11)$ \\
$\mathrm{Mn} 1-\mathrm{N} 3$ & $2.268(4)$ & $\mathrm{C} 36-\mathrm{C} 37$ & $1.402(12)$ \\
$\mathrm{Mn} 1-\mathrm{N} 5$ & $2.259(5)$ & $\mathrm{C} 36-\mathrm{H} 36$ & 0.9500 \\
$\mathrm{Mn} 1-\mathrm{N} 6$ & $2.212(4)$ & $\mathrm{C} 37-\mathrm{C} 38$ & $1.373(10)$ \\
$\mathrm{Mn} 1-\mathrm{N} 7$ & $2.260(5)$ & $\mathrm{C} 37-\mathrm{H} 37$ & 0.9500 \\
$\mathrm{Mn} 1-\mathrm{N} 105$ & $2.373(11)$ & $\mathrm{C} 38-\mathrm{C} 39$ & $1.394(10)$
\end{tabular}




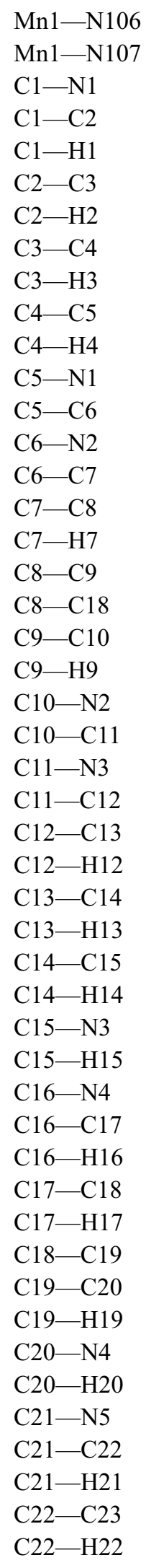

2.107 (10)

$2.090(16)$

$1.343(7)$

$1.371(8)$

0.9500

$1.387(8)$

0.9500

$1.374(8)$

0.9500

$1.388(7)$

0.9500

$1.352(6)$

$1.484(7)$

$1.338(6)$

$1.388(7)$

$1.388(7)$

0.9500

$1.393(7)$

$1.487(7)$

$1.380(7)$

0.9500

$1.343(6)$

$1.489(7)$

$1.346(6)$

1.389 (7)

$1.378(8)$

0.9500

$1.385(8)$

0.9500

$1.393(8)$

0.9500

1.334 (7)

0.9500

$1.315(8)$

$1.381(8)$

0.9500

$1.380(8)$

0.9500

$1.376(8)$

$1.393(8)$

0.9500

$1.322(9)$

0.9500

$1.336(7)$

$1.372(8)$

0.9500

1.377 (9)

0.9500
C $39-\mathrm{C} 40$

C39-H39

$\mathrm{C} 40-\mathrm{N} 8$

$\mathrm{C} 40-\mathrm{H} 40$

C121-N105

C121-C122

C121-H121

C122-C123

C122-H122

C123-C124

C123-H123

C124-C125

C124-H124

C125-N105

C125-C126

C126-N106

$\mathrm{C} 126-\mathrm{C} 127$

C127-C128

C127-H127

C129-C130

C129-C128

C129-H129

C130-N106

C130-C131

C131-N107

C131-C132

C132-C133

C132-H132

C133-C134

C133-H133

C134-C135

C134-H134

C135-N107

C135-H135

C128-C138

C136- 137

C136-N108

C136- H136

C137-C138

C137-H137

C138-C139

C139- $\mathrm{C} 140$

C139-H139

C140-N108

C140-H140

P1A-F4A

P1A-F1A

P1A-F5A

$1.384(9)$
0.9500
$1.336(10)$
0.9500
$1.336(7)$
$1.372(8)$
0.9500
$1.377(9)$
0.9500
$1.377(9)$
0.9500
$1.380(9)$
0.9500
$1.356(7)$
$1.481(8)$
$1.348(7)$
$1.382(7)$
$1.40(5)$
0.9500
$1.391(7)$
$1.47(5)$
0.9500
$1.336(7)$
$1.478(8)$
$1.351(6)$
$1.391(8)$
$1.374(9)$
0.9500
$1.375(8)$
0.9500
$1.368(9)$
0.9500
$1.343(7)$
0.9500
$1.46(5)$
1.3900
1.3900
0.9500
1.3900
0.9500
1.3900
1.3900
0.9500
1.3900
0.9500
$1.544(6)$
$1.564(7)$
$1.582(7)$




\begin{tabular}{|c|c|c|c|}
\hline $\mathrm{C} 23-\mathrm{C} 24$ & $1.377(9)$ & $\mathrm{P} 1 \mathrm{~A}-\mathrm{F} 6 \mathrm{~A}$ & $1.583(7)$ \\
\hline $\mathrm{C} 23-\mathrm{H} 23$ & 0.9500 & $\mathrm{P} 1 \mathrm{~A}-\mathrm{F} 2 \mathrm{~A}$ & $1.656(7)$ \\
\hline $\mathrm{C} 24-\mathrm{C} 25$ & $1.380(9)$ & $\mathrm{P} 1 \mathrm{~A}-\mathrm{F} 3 \mathrm{~A}$ & $1.669(7)$ \\
\hline $\mathrm{C} 24-\mathrm{H} 24$ & 0.9500 & $\mathrm{P} 1 \mathrm{~B}-\mathrm{F} 3 \mathrm{~B}$ & $1.542(11)$ \\
\hline $\mathrm{C} 25-\mathrm{N} 5$ & $1.356(6)$ & $\mathrm{P} 1 \mathrm{~B}-\mathrm{F} 2 \mathrm{~B}$ & $1.550(11)$ \\
\hline $\mathrm{C} 25-\mathrm{C} 26$ & $1.481(8)$ & $\mathrm{P} 1 \mathrm{~B}-\mathrm{F} 5 \mathrm{~B}$ & $1.581(11)$ \\
\hline $\mathrm{C} 26-\mathrm{N} 6$ & $1.348(7)$ & $\mathrm{P} 1 \mathrm{~B}-\mathrm{F} 1 \mathrm{~B}$ & $1.611(11)$ \\
\hline $\mathrm{C} 26-\mathrm{C} 27$ & $1.382(7)$ & $\mathrm{P} 1 \mathrm{~B}-\mathrm{F} 6 \mathrm{~B}$ & $1.656(14)$ \\
\hline $\mathrm{C} 27-\mathrm{C} 28$ & $1.391(10)$ & $\mathrm{P} 1 \mathrm{~B}-\mathrm{F} 4 \mathrm{~B}$ & $1.679(11)$ \\
\hline $\mathrm{C} 27-\mathrm{H} 27$ & 0.9500 & $\mathrm{P} 2 \mathrm{~A}-\mathrm{F} 9 \mathrm{~A}$ & $1.558(7)$ \\
\hline $\mathrm{C} 29-\mathrm{C} 28$ & $1.386(10)$ & $\mathrm{P} 2 \mathrm{~A}-\mathrm{F} 11 \mathrm{~A}$ & $1.565(6)$ \\
\hline $\mathrm{C} 29-\mathrm{C} 30$ & $1.391(7)$ & $\mathrm{P} 2 \mathrm{~A}-\mathrm{F} 8 \mathrm{~A}$ & $1.593(7)$ \\
\hline $\mathrm{C} 29-\mathrm{H} 29$ & 0.9500 & $\mathrm{P} 2 \mathrm{~A}-\mathrm{F} 12 \mathrm{~A}$ & $1.616(7)$ \\
\hline $\mathrm{C} 30-\mathrm{N} 6$ & $1.336(7)$ & $\mathrm{P} 2 \mathrm{~A}-\mathrm{F} 7 \mathrm{~A}$ & $1.627(7)$ \\
\hline $\mathrm{C} 30-\mathrm{C} 31$ & $1.477(8)$ & $\mathrm{P} 2 \mathrm{~A}-\mathrm{F} 10 \mathrm{~A}$ & $1.636(7)$ \\
\hline $\mathrm{C} 31-\mathrm{N} 7$ & $1.351(6)$ & $\mathrm{P} 2 \mathrm{~B}-\mathrm{F} 7 \mathrm{~B}$ & $1.484(11)$ \\
\hline $\mathrm{C} 31-\mathrm{C} 32$ & $1.391(8)$ & $\mathrm{P} 2 \mathrm{~B}-\mathrm{F} 10 \mathrm{~B}$ & $1.549(14)$ \\
\hline $\mathrm{C} 32-\mathrm{C} 33$ & $1.374(9)$ & $\mathrm{P} 2 \mathrm{~B}-\mathrm{F} 12 \mathrm{~B}$ & $1.573(11)$ \\
\hline C $32-\mathrm{H} 32$ & 0.9500 & $\mathrm{P} 2 \mathrm{~B}-\mathrm{F} 8 \mathrm{~B}$ & $1.615(12)$ \\
\hline $\mathrm{C} 33-\mathrm{C} 34$ & $1.375(8)$ & $\mathrm{P} 2 \mathrm{~B}-\mathrm{F} 11 \mathrm{~B}$ & $1.668(11)$ \\
\hline C33-H33 & 0.9500 & $\mathrm{P} 2 \mathrm{~B}-\mathrm{F} 9 \mathrm{~B}$ & $1.670(11)$ \\
\hline $\mathrm{C} 34-\mathrm{C} 35$ & $1.368(9)$ & $\mathrm{O} 1 \mathrm{~W}-\mathrm{O} 2 \mathrm{~W}$ & $0.693(13)$ \\
\hline C $34-\mathrm{H} 34$ & 0.9500 & $\mathrm{O} 1 \mathrm{~W}-\mathrm{O} 3 \mathrm{~W}$ & $1.41(3)$ \\
\hline $\mathrm{C} 35-\mathrm{N} 7$ & $1.343(7)$ & $\mathrm{O} 2 \mathrm{~W}-\mathrm{O} 3 \mathrm{~W}$ & $0.96(3)$ \\
\hline C35-H35 & 0.9500 & & \\
\hline $\mathrm{N} 1-\mathrm{Mn} 1-\mathrm{N} 2$ & $72.37(15)$ & $\mathrm{C} 29-\mathrm{C} 28-\mathrm{C} 38$ & $118.6(6)$ \\
\hline $\mathrm{N} 1-\mathrm{Mn} 1-\mathrm{N} 3$ & $143.36(15)$ & $\mathrm{C} 27-\mathrm{C} 28-\mathrm{C} 38$ & $122.3(6)$ \\
\hline $\mathrm{N} 1-\mathrm{Mn} 1-\mathrm{N} 5$ & $99.8(3)$ & $\mathrm{N} 8-\mathrm{C} 36-\mathrm{C} 37$ & $124.3(9)$ \\
\hline $\mathrm{N} 1-\mathrm{Mn} 1-\mathrm{N} 6$ & $111.3(3)$ & $\mathrm{N} 8-\mathrm{C} 36-\mathrm{H} 36$ & 117.9 \\
\hline $\mathrm{N} 1-\mathrm{Mn} 1-\mathrm{N} 7$ & $92.2(4)$ & $\mathrm{C} 37-\mathrm{C} 36-\mathrm{H} 36$ & 117.9 \\
\hline $\mathrm{N} 2-\mathrm{Mn} 1-\mathrm{N} 3$ & $72.21(15)$ & $\mathrm{C} 38-\mathrm{C} 37-\mathrm{C} 36$ & $118.0(7)$ \\
\hline $\mathrm{N} 2-\mathrm{Mn} 1-\mathrm{N} 5$ & $97.87(16)$ & $\mathrm{C} 38-\mathrm{C} 37-\mathrm{H} 37$ & 121.0 \\
\hline $\mathrm{N} 2-\mathrm{Mn} 1-\mathrm{N} 6$ & $169.95(18)$ & $\mathrm{C} 36-\mathrm{C} 37-\mathrm{H} 37$ & 121.0 \\
\hline $\mathrm{N} 2-\mathrm{Mn} 1-\mathrm{N} 7$ & $117.68(17)$ & $\mathrm{C} 37-\mathrm{C} 38-\mathrm{C} 39$ & $118.7(6)$ \\
\hline $\mathrm{N} 3-\mathrm{Mn} 1-\mathrm{N} 5$ & $94.0(3)$ & $\mathrm{C} 37-\mathrm{C} 38-\mathrm{C} 28$ & $121.9(7)$ \\
\hline N3-Mn1-N6 & $105.2(3)$ & $\mathrm{C} 39-\mathrm{C} 38-\mathrm{C} 28$ & $119.4(6)$ \\
\hline $\mathrm{N} 3-\mathrm{Mn} 1-\mathrm{N} 7$ & $96.0(4)$ & $\mathrm{C} 40-\mathrm{C} 39-\mathrm{C} 38$ & $118.5(7)$ \\
\hline $\mathrm{N} 5-\mathrm{Mn} 1-\mathrm{N} 6$ & $72.45(17)$ & $\mathrm{C} 40-\mathrm{C} 39-\mathrm{H} 39$ & 120.7 \\
\hline $\mathrm{N} 5-\mathrm{Mn} 1-\mathrm{N} 7$ & $144.44(17)$ & $\mathrm{C} 38-\mathrm{C} 39-\mathrm{H} 39$ & 120.7 \\
\hline $\mathrm{N} 6-\mathrm{Mn} 1-\mathrm{N} 7$ & $71.99(17)$ & $\mathrm{N} 8-\mathrm{C} 40-\mathrm{C} 39$ & $123.9(7)$ \\
\hline N107-Mn1-N106 & $77.6(5)$ & $\mathrm{N} 8-\mathrm{C} 40-\mathrm{H} 40$ & 118.1 \\
\hline $\mathrm{N} 107-\mathrm{Mn} 1-\mathrm{N} 2$ & $118.8(4)$ & $\mathrm{C} 39-\mathrm{C} 40-\mathrm{H} 40$ & 118.1 \\
\hline $\mathrm{N} 106-\mathrm{Mn} 1-\mathrm{N} 2$ & $163.6(3)$ & $\mathrm{C} 36-\mathrm{N} 8-\mathrm{C} 40$ & $116.5(7)$ \\
\hline N107-Mn1-N1 & $89(2)$ & $\mathrm{N} 105-\mathrm{C} 121-\mathrm{C} 122$ & $123.4(6)$ \\
\hline $\mathrm{N} 106-\mathrm{Mn} 1-\mathrm{N} 1$ & $110.1(14)$ & $\mathrm{N} 105-\mathrm{C} 121-\mathrm{H} 121$ & 118.3 \\
\hline $\mathrm{N} 107-\mathrm{Mn} 1-\mathrm{N} 3$ & $100(3)$ & $\mathrm{C} 122-\mathrm{C} 121-\mathrm{H} 121$ & 118.3 \\
\hline $\mathrm{N} 106-\mathrm{Mn} 1-\mathrm{N} 3$ & $106.5(14)$ & $\mathrm{C} 121-\mathrm{C} 122-\mathrm{C} 123$ & $118.0(6)$ \\
\hline
\end{tabular}




\begin{tabular}{|c|c|}
\hline N107-Mn1-N105 & $149.2(10)$ \\
\hline N106-Mn1-N105 & $72.0(3)$ \\
\hline N2-Mn1-N105 & $91.6(3)$ \\
\hline N1-Mn1-N105 & $96.8(16)$ \\
\hline N3-Mn1-N105 & $93.3(17)$ \\
\hline $\mathrm{N} 1-\mathrm{C} 1-\mathrm{C} 2$ & $122.4(5)$ \\
\hline $\mathrm{N} 1-\mathrm{C} 1-\mathrm{H} 1$ & 118.8 \\
\hline $\mathrm{C} 2-\mathrm{C} 1-\mathrm{H} 1$ & 118.8 \\
\hline $\mathrm{C} 1-\mathrm{C} 2-\mathrm{C} 3$ & $118.8(5)$ \\
\hline $\mathrm{C} 1-\mathrm{C} 2-\mathrm{H} 2$ & 120.6 \\
\hline $\mathrm{C} 3-\mathrm{C} 2-\mathrm{H} 2$ & 120.6 \\
\hline $\mathrm{C} 4-\mathrm{C} 3-\mathrm{C} 2$ & $119.2(5)$ \\
\hline $\mathrm{C} 4-\mathrm{C} 3-\mathrm{H} 3$ & 120.4 \\
\hline $\mathrm{C} 2-\mathrm{C} 3-\mathrm{H} 3$ & 120.4 \\
\hline $\mathrm{C} 3-\mathrm{C} 4-\mathrm{C} 5$ & $119.5(5)$ \\
\hline $\mathrm{C} 3-\mathrm{C} 4-\mathrm{H} 4$ & 120.3 \\
\hline $\mathrm{C} 5-\mathrm{C} 4-\mathrm{H} 4$ & 120.3 \\
\hline $\mathrm{N} 1-\mathrm{C} 5-\mathrm{C} 4$ & $121.0(5)$ \\
\hline $\mathrm{N} 1-\mathrm{C} 5-\mathrm{C} 6$ & $114.9(4)$ \\
\hline $\mathrm{C} 4-\mathrm{C} 5-\mathrm{C} 6$ & $124.1(4)$ \\
\hline $\mathrm{N} 2-\mathrm{C} 6-\mathrm{C} 7$ & $121.0(4)$ \\
\hline $\mathrm{N} 2-\mathrm{C} 6-\mathrm{C} 5$ & $114.7(4)$ \\
\hline $\mathrm{C} 7-\mathrm{C} 6-\mathrm{C} 5$ & $124.2(4)$ \\
\hline $\mathrm{C} 8-\mathrm{C} 7-\mathrm{C} 6$ & $119.9(5)$ \\
\hline $\mathrm{C} 8-\mathrm{C} 7-\mathrm{H} 7$ & 120.1 \\
\hline $\mathrm{C} 6-\mathrm{C} 7-\mathrm{H} 7$ & 120.1 \\
\hline $\mathrm{C} 7-\mathrm{C} 8-\mathrm{C} 9$ & $118.2(5)$ \\
\hline $\mathrm{C} 7-\mathrm{C} 8-\mathrm{C} 18$ & $121.8(5)$ \\
\hline $\mathrm{C} 9-\mathrm{C} 8-\mathrm{C} 18$ & $120.0(5)$ \\
\hline $\mathrm{C} 10-\mathrm{C} 9-\mathrm{C} 8$ & $119.3(5)$ \\
\hline $\mathrm{C} 10-\mathrm{C} 9-\mathrm{H} 9$ & 120.4 \\
\hline $\mathrm{C} 8-\mathrm{C} 9-\mathrm{H} 9$ & 120.4 \\
\hline $\mathrm{N} 2-\mathrm{C} 10-\mathrm{C} 9$ & $121.8(5)$ \\
\hline $\mathrm{N} 2-\mathrm{C} 10-\mathrm{C} 11$ & $114.1(4)$ \\
\hline $\mathrm{C} 9-\mathrm{C} 10-\mathrm{C} 11$ & $124.0(5)$ \\
\hline $\mathrm{N} 3-\mathrm{C} 11-\mathrm{C} 12$ & $121.4(5)$ \\
\hline $\mathrm{N} 3-\mathrm{C} 11-\mathrm{C} 10$ & $115.9(4)$ \\
\hline $\mathrm{C} 12-\mathrm{C} 11-\mathrm{C} 10$ & $122.7(5)$ \\
\hline $\mathrm{C} 13-\mathrm{C} 12-\mathrm{C} 11$ & $118.9(5)$ \\
\hline $\mathrm{C} 13-\mathrm{C} 12-\mathrm{H} 12$ & 120.6 \\
\hline $\mathrm{C} 11-\mathrm{C} 12-\mathrm{H} 12$ & 120.6 \\
\hline $\mathrm{C} 12-\mathrm{C} 13-\mathrm{C} 14$ & $119.9(5)$ \\
\hline $\mathrm{C} 12-\mathrm{C} 13-\mathrm{H} 13$ & 120.0 \\
\hline $\mathrm{C} 14-\mathrm{C} 13-\mathrm{H} 13$ & 120.0 \\
\hline $\mathrm{C} 13-\mathrm{C} 14-\mathrm{C} 15$ & $118.0(5)$ \\
\hline $\mathrm{C} 13-\mathrm{C} 14-\mathrm{H} 14$ & 121.0 \\
\hline $\mathrm{C} 15-\mathrm{C} 14-\mathrm{H} 14$ & 121.0 \\
\hline $\mathrm{N} 3-\mathrm{C} 15-\mathrm{C} 14$ & $122.3(5)$ \\
\hline
\end{tabular}

$149.2(10)$

$72.0(3)$

$96.8(16)$

$93.3(17)$

$122.4(5)$

118.8

118.8

$118.8(5)$

120.6

120.6

119.2 (5)

120.4

120.4

120.3

120.3

$121.0(5)$

114.9 (4)

$124.1(4)$

$121.0(4)$

114.7 (4)

124.2 (4)

120.1

$118.2(5)$

$121.8(5)$

$120.0(5)$

$119.3(5)$

120.4

120.4

$121.8(5)$

$114.1(4)$

$124.0(5)$

$121.4(5)$

$115.9(4)$

$122.7(5)$

120.6

120.6

$119.9(5)$

120.0

120.0

$118.0(5)$

121.0

$122.3(5)$
C121-C122-H122

$\mathrm{C} 123-\mathrm{C} 122-\mathrm{H} 122$

$\mathrm{C} 122-\mathrm{C} 123-\mathrm{C} 124$

C122-C123-H123

$\mathrm{C} 124-\mathrm{C} 123-\mathrm{H} 123$

$\mathrm{C} 123-\mathrm{C} 124-\mathrm{C} 125$

$\mathrm{C} 123-\mathrm{C} 124-\mathrm{H} 124$

$\mathrm{C} 125-\mathrm{C} 124-\mathrm{H} 124$

$\mathrm{N} 105-\mathrm{C} 125-\mathrm{C} 124$

$\mathrm{N} 105-\mathrm{C} 125-\mathrm{C} 126$

$\mathrm{C} 124-\mathrm{C} 125-\mathrm{C} 126$

N106-C126-C127

N106-C126-C125

$\mathrm{C} 127-\mathrm{C} 126-\mathrm{C} 125$

$\mathrm{C} 126-\mathrm{C} 127-\mathrm{C} 128$

$\mathrm{C} 126-\mathrm{C} 127-\mathrm{H} 127$

C128- C127-H127

$\mathrm{C} 130-\mathrm{C} 129-\mathrm{C} 128$

C130- C129-H129

C128- C129-H129

$\mathrm{N} 106-\mathrm{C} 130-\mathrm{C} 129$

N106-C130-C131

$\mathrm{C} 129-\mathrm{C} 130-\mathrm{C} 131$

N107-C131-C132

N107-C131-C130

$\mathrm{C} 132-\mathrm{C} 131-\mathrm{C} 130$

$\mathrm{C} 133-\mathrm{C} 132-\mathrm{C} 131$

C133- $132-\mathrm{H} 132$

C131-C132-H132

$\mathrm{C} 132-\mathrm{C} 133-\mathrm{C} 134$

$\mathrm{C} 132-\mathrm{C} 133-\mathrm{H} 133$

C134- C133-H133

C135-C134-C133

C135-C134-H134

C133-C134-H134

$\mathrm{N} 107-\mathrm{C} 135-\mathrm{C} 134$

N107-C135-H135

C134- C135-H135

C121-N105-C125

C121-N105-Mn1

C125-N105-Mn1

C130-N106-C126

C130-N106-Mn1

C126-N106-Mn1

C135-N107-C131

C135-N107-Mn1

C131-N107-Mn1

$\mathrm{C} 127-\mathrm{C} 128-\mathrm{C} 138$
121.0

121.0

$119.6(7)$

120.2

120.2

$119.6(6)$

120.2

120.2

$120.7(6)$

$116.0(5)$

$123.2(5)$

$120.5(6)$

$114.9(5)$

124.5 (6)

123 (2)

118.4

118.4

120.8 (19)

119.6

119.6

$121.0(6)$

115.7 (4)

$123.2(6)$

$121.7(5)$

$115.0(5)$

$123.2(5)$

118.8 (5)

120.6

120.6

119.7 (6)

120.2

120.2

$118.6(6)$

120.7

120.7

123.1 (6)

118.5

118.5

$118.6(5)$

$128.0(5)$

113.4 (4)

$120.8(5)$

115.5 (5)

$123.7(5)$

$118.0(5)$

125.5 (13)

$115.6(6)$

$125(3)$ 


\begin{tabular}{|c|c|}
\hline $\mathrm{N} 3-\mathrm{C} 15-\mathrm{H} 15$ & 118.8 \\
\hline $\mathrm{C} 14-\mathrm{C} 15-\mathrm{H} 15$ & 118.8 \\
\hline $\mathrm{N} 4-\mathrm{C} 16-\mathrm{C} 17$ & $124.0(6)$ \\
\hline $\mathrm{N} 4-\mathrm{C} 16-\mathrm{H} 16$ & 118.0 \\
\hline $\mathrm{C} 17-\mathrm{C} 16-\mathrm{H} 16$ & 118.0 \\
\hline $\mathrm{C} 18-\mathrm{C} 17-\mathrm{C} 16$ & $120.1(6)$ \\
\hline $\mathrm{C} 18-\mathrm{C} 17-\mathrm{H} 17$ & 119.9 \\
\hline $\mathrm{C} 16-\mathrm{C} 17-\mathrm{H} 17$ & 119.9 \\
\hline $\mathrm{C} 19-\mathrm{C} 18-\mathrm{C} 17$ & $115.9(5)$ \\
\hline $\mathrm{C} 19-\mathrm{C} 18-\mathrm{C} 8$ & $121.3(5)$ \\
\hline $\mathrm{C} 17-\mathrm{C} 18-\mathrm{C} 8$ & $122.5(5)$ \\
\hline $\mathrm{C} 18-\mathrm{C} 19-\mathrm{C} 20$ & $120.0(6)$ \\
\hline $\mathrm{C} 18-\mathrm{C} 19-\mathrm{H} 19$ & 120.0 \\
\hline $\mathrm{C} 20-\mathrm{C} 19-\mathrm{H} 19$ & 120.0 \\
\hline $\mathrm{N} 4-\mathrm{C} 20-\mathrm{C} 19$ & $123.5(6)$ \\
\hline $\mathrm{N} 4-\mathrm{C} 20-\mathrm{H} 20$ & 118.3 \\
\hline $\mathrm{C} 19-\mathrm{C} 20-\mathrm{H} 20$ & 118.3 \\
\hline $\mathrm{C} 1-\mathrm{N} 1-\mathrm{C} 5$ & $119.0(4)$ \\
\hline $\mathrm{C} 1-\mathrm{N} 1-\mathrm{Mn} 1$ & $123.2(4)$ \\
\hline $\mathrm{C} 5-\mathrm{N} 1-\mathrm{Mn} 1$ & $117.7(3)$ \\
\hline $\mathrm{C} 6-\mathrm{N} 2-\mathrm{C} 10$ & $119.9(4)$ \\
\hline $\mathrm{C} 6-\mathrm{N} 2-\mathrm{Mn} 1$ & $119.6(3)$ \\
\hline $\mathrm{C} 10-\mathrm{N} 2-\mathrm{Mn} 1$ & $120.2(3)$ \\
\hline $\mathrm{C} 15-\mathrm{N} 3-\mathrm{C} 11$ & $119.4(4)$ \\
\hline $\mathrm{C} 15-\mathrm{N} 3-\mathrm{Mn} 1$ & $123.8(3)$ \\
\hline $\mathrm{C} 11-\mathrm{N} 3-\mathrm{Mn} 1$ & $116.8(3)$ \\
\hline $\mathrm{C} 16-\mathrm{N} 4-\mathrm{C} 20$ & $116.4(5)$ \\
\hline $\mathrm{N} 5-\mathrm{C} 21-\mathrm{C} 22$ & $123.4(5)$ \\
\hline $\mathrm{N} 5-\mathrm{C} 21-\mathrm{H} 21$ & 118.3 \\
\hline $\mathrm{C} 22-\mathrm{C} 21-\mathrm{H} 21$ & 118.3 \\
\hline $\mathrm{C} 21-\mathrm{C} 22-\mathrm{C} 23$ & $118.0(6)$ \\
\hline $\mathrm{C} 21-\mathrm{C} 22-\mathrm{H} 22$ & 121.0 \\
\hline $\mathrm{C} 23-\mathrm{C} 22-\mathrm{H} 22$ & 121.0 \\
\hline $\mathrm{C} 22-\mathrm{C} 23-\mathrm{C} 24$ & $119.6(6)$ \\
\hline $\mathrm{C} 22-\mathrm{C} 23-\mathrm{H} 23$ & 120.2 \\
\hline $\mathrm{C} 24-\mathrm{C} 23-\mathrm{H} 23$ & 120.2 \\
\hline $\mathrm{C} 23-\mathrm{C} 24-\mathrm{C} 25$ & $119.7(6)$ \\
\hline $\mathrm{C} 23-\mathrm{C} 24-\mathrm{H} 24$ & 120.2 \\
\hline $\mathrm{C} 25-\mathrm{C} 24-\mathrm{H} 24$ & 120.2 \\
\hline $\mathrm{N} 5-\mathrm{C} 25-\mathrm{C} 24$ & $120.8(5)$ \\
\hline $\mathrm{N} 5-\mathrm{C} 25-\mathrm{C} 26$ & $116.0(5)$ \\
\hline $\mathrm{C} 24-\mathrm{C} 25-\mathrm{C} 26$ & $123.2(5)$ \\
\hline $\mathrm{N} 6-\mathrm{C} 26-\mathrm{C} 27$ & $120.5(6)$ \\
\hline $\mathrm{N} 6-\mathrm{C} 26-\mathrm{C} 25$ & $114.9(4)$ \\
\hline $\mathrm{C} 27-\mathrm{C} 26-\mathrm{C} 25$ & $124.5(5)$ \\
\hline $\mathrm{C} 26-\mathrm{C} 27-\mathrm{C} 28$ & $119.4(6)$ \\
\hline $\mathrm{C} 26-\mathrm{C} 27-\mathrm{H} 27$ & 120.3 \\
\hline $\mathrm{C} 28-\mathrm{C} 27-\mathrm{H} 27$ & 120.3 \\
\hline
\end{tabular}

\begin{tabular}{|c|c|}
\hline $\mathrm{C} 127-\mathrm{C} 128-\mathrm{C} 129$ & $114(3)$ \\
\hline $\mathrm{C} 138-\mathrm{C} 128-\mathrm{C} 129$ & $121(3)$ \\
\hline $\mathrm{C} 137-\mathrm{C} 136-\mathrm{N} 108$ & 120.0 \\
\hline $\mathrm{C} 137-\mathrm{C} 136-\mathrm{H} 136$ & 120.0 \\
\hline $\mathrm{N} 108-\mathrm{C} 136-\mathrm{H} 136$ & 120.0 \\
\hline $\mathrm{C} 136-\mathrm{C} 137-\mathrm{C} 138$ & 120.0 \\
\hline $\mathrm{C} 136-\mathrm{C} 137-\mathrm{H} 137$ & 120.0 \\
\hline $\mathrm{C} 138-\mathrm{C} 137-\mathrm{H} 137$ & 120.0 \\
\hline $\mathrm{C} 139-\mathrm{C} 138-\mathrm{C} 137$ & 120.0 \\
\hline $\mathrm{C} 139-\mathrm{C} 138-\mathrm{C} 128$ & $118(2)$ \\
\hline $\mathrm{C} 137-\mathrm{C} 138-\mathrm{C} 128$ & $122(2)$ \\
\hline $\mathrm{C} 140-\mathrm{C} 139-\mathrm{C} 138$ & 120.0 \\
\hline $\mathrm{C} 140-\mathrm{C} 139-\mathrm{H} 139$ & 120.0 \\
\hline $\mathrm{C} 138-\mathrm{C} 139-\mathrm{H} 139$ & 120.0 \\
\hline $\mathrm{C} 139-\mathrm{C} 140-\mathrm{N} 108$ & 120.0 \\
\hline $\mathrm{C} 139-\mathrm{C} 140-\mathrm{H} 140$ & 120.0 \\
\hline $\mathrm{N} 108-\mathrm{C} 140-\mathrm{H} 140$ & 120.0 \\
\hline $\mathrm{C} 140-\mathrm{N} 108-\mathrm{C} 136$ & 120.0 \\
\hline $\mathrm{F} 4 \mathrm{~A}-\mathrm{P} 1 \mathrm{~A}-\mathrm{F} 1 \mathrm{~A}$ & $96.0(4)$ \\
\hline $\mathrm{F} 4 \mathrm{~A}-\mathrm{P} 1 \mathrm{~A}-\mathrm{F} 5 \mathrm{~A}$ & $90.4(4)$ \\
\hline $\mathrm{F} 1 \mathrm{~A}-\mathrm{P} 1 \mathrm{~A}-\mathrm{F} 5 \mathrm{~A}$ & $94.1(5)$ \\
\hline $\mathrm{F} 4 \mathrm{~A}-\mathrm{P} 1 \mathrm{~A}-\mathrm{F} 6 \mathrm{~A}$ & $91.7(4)$ \\
\hline $\mathrm{F} 1 \mathrm{~A}-\mathrm{P} 1 \mathrm{~A}-\mathrm{F} 6 \mathrm{~A}$ & $92.7(5)$ \\
\hline $\mathrm{F} 5 \mathrm{~A}-\mathrm{P} 1 \mathrm{~A}-\mathrm{F} 6 \mathrm{~A}$ & $172.7(4)$ \\
\hline $\mathrm{F} 4 \mathrm{~A}-\mathrm{P} 1 \mathrm{~A}-\mathrm{F} 2 \mathrm{~A}$ & $171.2(4)$ \\
\hline $\mathrm{F} 1 \mathrm{~A}-\mathrm{P} 1 \mathrm{~A}-\mathrm{F} 2 \mathrm{~A}$ & $92.8(4)$ \\
\hline $\mathrm{F} 5 \mathrm{~A}-\mathrm{P} 1 \mathrm{~A}-\mathrm{F} 2 \mathrm{~A}$ & $89.2(4)$ \\
\hline $\mathrm{F} 6 \mathrm{~A}-\mathrm{P} 1 \mathrm{~A}-\mathrm{F} 2 \mathrm{~A}$ & $87.7(4)$ \\
\hline $\mathrm{F} 4 \mathrm{~A}-\mathrm{P} 1 \mathrm{~A}-\mathrm{F} 3 \mathrm{~A}$ & $89.6(4)$ \\
\hline $\mathrm{F} 1 \mathrm{~A}-\mathrm{P} 1 \mathrm{~A}-\mathrm{F} 3 \mathrm{~A}$ & 173.9 \\
\hline $\mathrm{F} 5 \mathrm{~A}-\mathrm{P} 1 \mathrm{~A}-\mathrm{F} 3 \mathrm{~A}$ & $83.5(4)$ \\
\hline $\mathrm{F} 6 \mathrm{~A}-\mathrm{P} 1 \mathrm{~A}-\mathrm{F} 3 \mathrm{~A}$ & $89.5(4)$ \\
\hline $\mathrm{F} 2 \mathrm{~A}-\mathrm{P} 1 \mathrm{~A}-\mathrm{F} 3 \mathrm{~A}$ & $81.5(3)$ \\
\hline $\mathrm{F} 3 \mathrm{~B}-\mathrm{P} 1 \mathrm{~B}-\mathrm{F} 2 \mathrm{~B}$ & $98.1(7)$ \\
\hline $\mathrm{F} 3 \mathrm{~B}-\mathrm{P} 1 \mathrm{~B}-\mathrm{F} 5 \mathrm{~B}$ & $96.9(7)$ \\
\hline $\mathrm{F} 2 \mathrm{~B}-\mathrm{P} 1 \mathrm{~B}-\mathrm{F} 5 \mathrm{~B}$ & $97.7(7)$ \\
\hline $\mathrm{F} 3 \mathrm{~B}-\mathrm{P} 1 \mathrm{~B}-\mathrm{F} 1 \mathrm{~B}$ & $175.5(8)$ \\
\hline $\mathrm{F} 2 \mathrm{~B}-\mathrm{P} 1 \mathrm{~B}-\mathrm{F} 1 \mathrm{~B}$ & $85.5(7)$ \\
\hline $\mathrm{F} 5 \mathrm{~B}-\mathrm{P} 1 \mathrm{~B}-\mathrm{F} 1 \mathrm{~B}$ & $85.3(9)$ \\
\hline $\mathrm{F} 3 \mathrm{~B}-\mathrm{P} 1 \mathrm{~B}-\mathrm{F} 6 \mathrm{~B}$ & $89.6(7)$ \\
\hline $\mathrm{F} 2 \mathrm{~B}-\mathrm{P} 1 \mathrm{~B}-\mathrm{F} 6 \mathrm{~B}$ & $94.7(7)$ \\
\hline $\mathrm{F} 5 \mathrm{~B}-\mathrm{P} 1 \mathrm{~B}-\mathrm{F} 6 \mathrm{~B}$ & $165.0(8)$ \\
\hline $\mathrm{F} 1 \mathrm{~B}-\mathrm{P} 1 \mathrm{~B}-\mathrm{F} 6 \mathrm{~B}$ & $87.3(9)$ \\
\hline $\mathrm{F} 3 \mathrm{~B}-\mathrm{P} 1 \mathrm{~B}-\mathrm{F} 4 \mathrm{~B}$ & $90.3(7)$ \\
\hline $\mathrm{F} 2 \mathrm{~B}-\mathrm{P} 1 \mathrm{~B}-\mathrm{F} 4 \mathrm{~B}$ & $171.3(7)$ \\
\hline $\mathrm{F} 5 \mathrm{~B}-\mathrm{P} 1 \mathrm{~B}-\mathrm{F} 4 \mathrm{~B}$ & $83.5(7)$ \\
\hline $\mathrm{F} 1 \mathrm{~B}-\mathrm{P} 1 \mathrm{~B}-\mathrm{F} 4 \mathrm{~B}$ & $86.0(7)$ \\
\hline $\mathrm{F} 6 \mathrm{~B}-\mathrm{P} 1 \mathrm{~B}-\mathrm{F} 4 \mathrm{~B}$ & $82.9(7)$ \\
\hline
\end{tabular}




\begin{tabular}{|c|c|}
\hline $\mathrm{C} 28-\mathrm{C} 29-\mathrm{C} 30$ & $118.9(6)$ \\
\hline $\mathrm{C} 28-\mathrm{C} 29-\mathrm{H} 29$ & 120.5 \\
\hline $\mathrm{C} 30-\mathrm{C} 29-\mathrm{H} 29$ & 120.5 \\
\hline $\mathrm{N} 6-\mathrm{C} 30-\mathrm{C} 29$ & $121.1(5)$ \\
\hline N6-C30-C31 & $115.7(4)$ \\
\hline $\mathrm{C} 29-\mathrm{C} 30-\mathrm{C} 31$ & $123.2(5)$ \\
\hline $\mathrm{N} 7-\mathrm{C} 31-\mathrm{C} 32$ & $121.7(5)$ \\
\hline $\mathrm{N} 7-\mathrm{C} 31-\mathrm{C} 30$ & $115.0(5)$ \\
\hline $\mathrm{C} 32-\mathrm{C} 31-\mathrm{C} 30$ & $123.2(5)$ \\
\hline $\mathrm{C} 33-\mathrm{C} 32-\mathrm{C} 31$ & $118.8(5)$ \\
\hline $\mathrm{C} 33-\mathrm{C} 32-\mathrm{H} 32$ & 120.6 \\
\hline $\mathrm{C} 31-\mathrm{C} 32-\mathrm{H} 32$ & 120.6 \\
\hline $\mathrm{C} 32-\mathrm{C} 33-\mathrm{C} 34$ & $119.7(6)$ \\
\hline $\mathrm{C} 32-\mathrm{C} 33-\mathrm{H} 33$ & 120.1 \\
\hline $\mathrm{C} 34-\mathrm{C} 33-\mathrm{H} 33$ & 120.1 \\
\hline $\mathrm{C} 35-\mathrm{C} 34-\mathrm{C} 33$ & $118.7(6)$ \\
\hline $\mathrm{C} 35-\mathrm{C} 34-\mathrm{H} 34$ & 120.7 \\
\hline $\mathrm{C} 33-\mathrm{C} 34-\mathrm{H} 34$ & 120.7 \\
\hline $\mathrm{N} 7-\mathrm{C} 35-\mathrm{C} 34$ & $123.1(5)$ \\
\hline N7-C35-H35 & 118.4 \\
\hline C34-C35-H35 & 118.4 \\
\hline $\mathrm{C} 21-\mathrm{N} 5-\mathrm{C} 25$ & $118.6(5)$ \\
\hline $\mathrm{C} 21-\mathrm{N} 5-\mathrm{Mn} 1$ & $124.3(4)$ \\
\hline $\mathrm{C} 25-\mathrm{N} 5-\mathrm{Mn} 1$ & $117.1(4)$ \\
\hline $\mathrm{C} 30-\mathrm{N} 6-\mathrm{C} 26$ & $120.8(4)$ \\
\hline $\mathrm{C} 30-\mathrm{N} 6-\mathrm{Mn} 1$ & $119.5(3)$ \\
\hline $\mathrm{C} 26-\mathrm{N} 6-\mathrm{Mn} 1$ & $119.6(4)$ \\
\hline $\mathrm{C} 35-\mathrm{N} 7-\mathrm{C} 31$ & $118.0(5)$ \\
\hline $\mathrm{C} 35-\mathrm{N} 7-\mathrm{Mn} 1$ & $124.3(4)$ \\
\hline $\mathrm{C} 31-\mathrm{N} 7-\mathrm{Mn} 1$ & $117.6(4)$ \\
\hline $\mathrm{C} 29-\mathrm{C} 28-\mathrm{C} 27$ & $119.1(6)$ \\
\hline $\mathrm{N} 1-\mathrm{C} 1-\mathrm{C} 2-\mathrm{C} 3$ & $-1.5(10)$ \\
\hline $\mathrm{C} 1-\mathrm{C} 2-\mathrm{C} 3-\mathrm{C} 4$ & $1.1(10)$ \\
\hline $\mathrm{C} 2-\mathrm{C} 3-\mathrm{C} 4-\mathrm{C} 5$ & $0.1(10)$ \\
\hline $\mathrm{C} 3-\mathrm{C} 4-\mathrm{C} 5-\mathrm{N} 1$ & $-1.1(9)$ \\
\hline $\mathrm{C} 3-\mathrm{C} 4-\mathrm{C} 5-\mathrm{C} 6$ & $177.0(5)$ \\
\hline $\mathrm{N} 1-\mathrm{C} 5-\mathrm{C} 6-\mathrm{N} 2$ & $2.3(7)$ \\
\hline $\mathrm{C} 4-\mathrm{C} 5-\mathrm{C} 6-\mathrm{N} 2$ & $-175.9(5)$ \\
\hline $\mathrm{N} 1-\mathrm{C} 5-\mathrm{C} 6-\mathrm{C} 7$ & $179.3(5)$ \\
\hline $\mathrm{C} 4-\mathrm{C} 5-\mathrm{C} 6-\mathrm{C} 7$ & $1.1(8)$ \\
\hline $\mathrm{N} 2-\mathrm{C} 6-\mathrm{C} 7-\mathrm{C} 8$ & $-0.7(8)$ \\
\hline $\mathrm{C} 5-\mathrm{C} 6-\mathrm{C} 7-\mathrm{C} 8$ & $-177.5(5)$ \\
\hline $\mathrm{C} 6-\mathrm{C} 7-\mathrm{C} 8-\mathrm{C} 9$ & $-0.3(8)$ \\
\hline $\mathrm{C} 6-\mathrm{C} 7-\mathrm{C} 8-\mathrm{C} 18$ & $178.3(5)$ \\
\hline $\mathrm{C} 7-\mathrm{C} 8-\mathrm{C} 9-\mathrm{C} 10$ & $1.2(8)$ \\
\hline $\mathrm{C} 18-\mathrm{C} 8-\mathrm{C} 9-\mathrm{C} 10$ & $-177.5(5)$ \\
\hline $\mathrm{C} 8-\mathrm{C} 9-\mathrm{C} 10-\mathrm{N} 2$ & $-1.2(8)$ \\
\hline
\end{tabular}

$\begin{array}{ll}\text { F9A-P2A-F11A } & 100.5(4) \\ \text { F9A-P2A-F8A } & 88.8(4) \\ \text { F11A-P2A-F8A } & 91.9(4) \\ \text { F9A-P2A-F12A } & 87.5(4) \\ \text { F11A-P2A-F12A } & 171.9(4) \\ \text { F8A-P2A-F12A } & 89.2(4) \\ \text { F9A-P2A-F7A } & 173.8(4) \\ \text { F11A-P2A-F7A } & 85.1(4) \\ \text { F8A-P2A-F7A } & 88.5(4) \\ \text { F12A-P2A-F7A } & 86.9(4) \\ \text { F9A-P2A-F10A } & 79.3(4) \\ \text { F11A-P2A-F10A } & 85.8(4) \\ \text { F8A-P2A-F10A } & 167.3(4) \\ \text { F12A-P2A-F10A } & 94.8(4) \\ \text { F7A-P2A-F10A } & 103.7(4) \\ \text { F7B-P2B-F10B } & 75.3(7) \\ \text { F7B-P2B-F12B } & 107.1(7) \\ \text { F10B-P2B-F12B } & 75.1(8) \\ \text { F7B-P2B-F8B } & 94.6(9) \\ \text { F10B-P2B-F8B } & 157.7(8) \\ \text { F12B-P2B-F8B } & 89.4(8) \\ \text { F7B-P2B-F11B } & 84.9(7) \\ \text { F10B-P2B-F11B } & 109.5(7) \\ \text { F12B-P2B-F11B } & 168.1(7) \\ \text { F8B-P2B-F11B } & 89.0(7) \\ \text { F7B-P2B-F9B } & 170.8(7) \\ \text { F10B-P2B-F9B } & 105.9(7) \\ \text { F12B-P2B-F9B } & 82.0(6) \\ \text { F8B-P2B-F9B } & 87.3(8) \\ \text { F11B-P2B-F9B } & 86.1(7) \\ \text { O1W-O2W-O3W } & 116(3) \\ \text { C31-C30-N6-C26 } & 178.8(8) \\ \text { C29-C30-N6-Mn1 } & 173.7(7) \\ \text { C31-C30-N6-Mn1 } & -5.2(9) \\ \text { C27-C26-N6-C30 } & -0.7(12) \\ \text { C25-C26-N6-C30 } & 177.3(7) \\ \text { C27-C26-N6-Mn1 } & -176.8(7) \\ \text { C25-C26-N6-Mn1 } & 1.3(10) \\ \text { C34-C35-N7-C31 } & 0(2) \\ \text { C34-C35-N7-Mn1 } & -177.4(15) \\ \text { C32-C31-N7-C35 } & -1.0(15) \\ \text { C30-C31-N7-C35 } & -178.2(12) \\ \text { C32-C31-N7-Mn1 } & 177.0(9) \\ \text { C30-C31-N7-Mn1 } & -0.3(10) \\ \text { C30-C29-C28-C27 } & 0.4(10) \\ \text { C30-C29-C28-C38 } & -179.3(7) \\ \text { C26-C27-C28-C29 } & -3.3(1) \\ & \end{array}$




\begin{tabular}{|c|c|}
\hline $\mathrm{C} 8-\mathrm{C} 9-\mathrm{C} 10-\mathrm{C} 11$ & $175.0(5)$ \\
\hline $\mathrm{N} 2-\mathrm{C} 10-\mathrm{C} 11-\mathrm{N} 3$ & $-5.8(7)$ \\
\hline $\mathrm{C} 9-\mathrm{C} 10-\mathrm{C} 11-\mathrm{N} 3$ & $177.8(5)$ \\
\hline $\mathrm{N} 2-\mathrm{C} 10-\mathrm{C} 11-\mathrm{C} 12$ & $172.5(5)$ \\
\hline $\mathrm{C} 9-\mathrm{C} 10-\mathrm{C} 11-\mathrm{C} 12$ & $-3.9(9)$ \\
\hline $\mathrm{N} 3-\mathrm{C} 11-\mathrm{C} 12-\mathrm{C} 13$ & $1.9(9)$ \\
\hline $\mathrm{C} 10-\mathrm{C} 11-\mathrm{C} 12-\mathrm{C} 13$ & $-176.3(6)$ \\
\hline $\mathrm{C} 11-\mathrm{C} 12-\mathrm{C} 13-\mathrm{C} 14$ & $-2.0(10)$ \\
\hline $\mathrm{C} 12-\mathrm{C} 13-\mathrm{C} 14-\mathrm{C} 15$ & $0.7(10)$ \\
\hline $\mathrm{C} 13-\mathrm{C} 14-\mathrm{C} 15-\mathrm{N} 3$ & $0.6(10)$ \\
\hline $\mathrm{N} 4-\mathrm{C} 16-\mathrm{C} 17-\mathrm{C} 18$ & $-1.4(12)$ \\
\hline $\mathrm{C} 16-\mathrm{C} 17-\mathrm{C} 18-\mathrm{C} 19$ & $-1.5(10)$ \\
\hline $\mathrm{C} 16-\mathrm{C} 17-\mathrm{C} 18-\mathrm{C} 8$ & $-176.6(6)$ \\
\hline $\mathrm{C} 7-\mathrm{C} 8-\mathrm{C} 18-\mathrm{C} 19$ & $-155.2(6)$ \\
\hline $\mathrm{C} 9-\mathrm{C} 8-\mathrm{C} 18-\mathrm{C} 19$ & $23.4(8)$ \\
\hline $\mathrm{C} 7-\mathrm{C} 8-\mathrm{C} 18-\mathrm{C} 17$ & $19.7(9)$ \\
\hline $\mathrm{C} 9-\mathrm{C} 8-\mathrm{C} 18-\mathrm{C} 17$ & $-161.7(6)$ \\
\hline $\mathrm{C} 17-\mathrm{C} 18-\mathrm{C} 19-\mathrm{C} 20$ & $2.1(10)$ \\
\hline $\mathrm{C} 8-\mathrm{C} 18-\mathrm{C} 19-\mathrm{C} 20$ & $177.3(6)$ \\
\hline $\mathrm{C} 18-\mathrm{C} 19-\mathrm{C} 20-\mathrm{N} 4$ & $0.1(12)$ \\
\hline $\mathrm{C} 2-\mathrm{C} 1-\mathrm{N} 1-\mathrm{C} 5$ & $0.6(8)$ \\
\hline $\mathrm{C} 2-\mathrm{C} 1-\mathrm{N} 1-\mathrm{Mn} 1$ & $178.4(5)$ \\
\hline $\mathrm{C} 4-\mathrm{C} 5-\mathrm{N} 1-\mathrm{C} 1$ & $0.8(8)$ \\
\hline $\mathrm{C} 6-\mathrm{C} 5-\mathrm{N} 1-\mathrm{C} 1$ & $-177.5(5)$ \\
\hline $\mathrm{C} 4-\mathrm{C} 5-\mathrm{N} 1-\mathrm{Mn} 1$ & $-177.2(4)$ \\
\hline $\mathrm{C} 6-\mathrm{C} 5-\mathrm{N} 1-\mathrm{Mn} 1$ & $4.5(6)$ \\
\hline $\mathrm{C} 7-\mathrm{C} 6-\mathrm{N} 2-\mathrm{C} 10$ & $0.7(8)$ \\
\hline $\mathrm{C} 5-\mathrm{C} 6-\mathrm{N} 2-\mathrm{C} 10$ & $177.8(5)$ \\
\hline $\mathrm{C} 7-\mathrm{C} 6-\mathrm{N} 2-\mathrm{Mn} 1$ & $174.5(4)$ \\
\hline $\mathrm{C} 5-\mathrm{C} 6-\mathrm{N} 2-\mathrm{Mn} 1$ & $-8.3(6)$ \\
\hline $\mathrm{C} 9-\mathrm{C} 10-\mathrm{N} 2-\mathrm{C} 6$ & $0.2(8)$ \\
\hline $\mathrm{C} 11-\mathrm{C} 10-\mathrm{N} 2-\mathrm{C} 6$ & $-176.3(5)$ \\
\hline $\mathrm{C} 9-\mathrm{C} 10-\mathrm{N} 2-\mathrm{Mn} 1$ & $-173.6(4)$ \\
\hline $\mathrm{C} 11-\mathrm{C} 10-\mathrm{N} 2-\mathrm{Mn} 1$ & $9.9(6)$ \\
\hline $\mathrm{C} 14-\mathrm{C} 15-\mathrm{N} 3-\mathrm{C} 11$ & $-0.7(9)$ \\
\hline $\mathrm{C} 14-\mathrm{C} 15-\mathrm{N} 3-\mathrm{Mn} 1$ & $177.6(5)$ \\
\hline $\mathrm{C} 12-\mathrm{C} 11-\mathrm{N} 3-\mathrm{C} 15$ & $-0.6(9)$ \\
\hline $\mathrm{C} 10-\mathrm{C} 11-\mathrm{N} 3-\mathrm{C} 15$ & $177.7(5)$ \\
\hline $\mathrm{C} 12-\mathrm{C} 11-\mathrm{N} 3-\mathrm{Mn} 1$ & $-179.0(5)$ \\
\hline $\mathrm{C} 10-\mathrm{C} 11-\mathrm{N} 3-\mathrm{Mn} 1$ & $-0.6(6)$ \\
\hline $\mathrm{C} 17-\mathrm{C} 16-\mathrm{N} 4-\mathrm{C} 20$ & $3.5(12)$ \\
\hline $\mathrm{C} 19-\mathrm{C} 20-\mathrm{N} 4-\mathrm{C} 16$ & $-2.9(11)$ \\
\hline $\mathrm{N} 5-\mathrm{C} 21-\mathrm{C} 22-\mathrm{C} 23$ & $-0.4(18)$ \\
\hline $\mathrm{C} 21-\mathrm{C} 22-\mathrm{C} 23-\mathrm{C} 24$ & $0.0(16)$ \\
\hline $\mathrm{C} 22-\mathrm{C} 23-\mathrm{C} 24-\mathrm{C} 25$ & $0.4(16)$ \\
\hline $\mathrm{C} 23-\mathrm{C} 24-\mathrm{C} 25-\mathrm{N} 5$ & $-0.5(15)$ \\
\hline $\mathrm{C} 23-\mathrm{C} 24-\mathrm{C} 25-\mathrm{C} 26$ & $177.4(10)$ \\
\hline $\mathrm{N} 5-\mathrm{C} 25-\mathrm{C} 26-\mathrm{N} 6$ & $-0.8(10)$ \\
\hline
\end{tabular}

\begin{tabular}{|c|c|}
\hline $\mathrm{C} 26-\mathrm{C} 27-\mathrm{C} 28-\mathrm{C} 38$ & $176.4(7)$ \\
\hline $\mathrm{N} 8-\mathrm{C} 36-\mathrm{C} 37-\mathrm{C} 38$ & $1.3(13)$ \\
\hline $\mathrm{C} 36-\mathrm{C} 37-\mathrm{C} 38-\mathrm{C} 39$ & $-1.7(11)$ \\
\hline $\mathrm{C} 36-\mathrm{C} 37-\mathrm{C} 38-\mathrm{C} 28$ & $176.3(7)$ \\
\hline $\mathrm{C} 29-\mathrm{C} 28-\mathrm{C} 38-\mathrm{C} 37$ & $-135.8(8)$ \\
\hline $\mathrm{C} 27-\mathrm{C} 28-\mathrm{C} 38-\mathrm{C} 37$ & $44.4(10)$ \\
\hline $\mathrm{C} 29-\mathrm{C} 28-\mathrm{C} 38-\mathrm{C} 39$ & $42.1(9)$ \\
\hline $\mathrm{C} 27-\mathrm{C} 28-\mathrm{C} 38-\mathrm{C} 39$ & $-137.6(7)$ \\
\hline $\mathrm{C} 37-\mathrm{C} 38-\mathrm{C} 39-\mathrm{C} 40$ & $0.4(11)$ \\
\hline $\mathrm{C} 28-\mathrm{C} 38-\mathrm{C} 39-\mathrm{C} 40$ & $-177.6(6)$ \\
\hline $\mathrm{C} 38-\mathrm{C} 39-\mathrm{C} 40-\mathrm{N} 8$ & $1.6(11)$ \\
\hline $\mathrm{C} 37-\mathrm{C} 36-\mathrm{N} 8-\mathrm{C} 40$ & $0.5(13)$ \\
\hline $\mathrm{C} 39-\mathrm{C} 40-\mathrm{N} 8-\mathrm{C} 36$ & $-1.9(12)$ \\
\hline $\mathrm{N} 105-\mathrm{C} 121-\mathrm{C} 122-\mathrm{C} 123$ & $1(11)$ \\
\hline $\mathrm{C} 121-\mathrm{C} 122-\mathrm{C} 123-\mathrm{C} 124$ & $-3(8)$ \\
\hline $\mathrm{C} 122-\mathrm{C} 123-\mathrm{C} 124-\mathrm{C} 125$ & $2(9)$ \\
\hline $\mathrm{C} 123-\mathrm{C} 124-\mathrm{C} 125-\mathrm{N} 105$ & $1(8)$ \\
\hline $\mathrm{C} 123-\mathrm{C} 124-\mathrm{C} 125-\mathrm{C} 126$ & $179(6)$ \\
\hline $\mathrm{N} 105-\mathrm{C} 125-\mathrm{C} 126-\mathrm{N} 106$ & $2(5)$ \\
\hline $\mathrm{C} 124-\mathrm{C} 125-\mathrm{C} 126-\mathrm{N} 106$ & $-175(5)$ \\
\hline $\mathrm{N} 105-\mathrm{C} 125-\mathrm{C} 126-\mathrm{C} 127$ & $-175(5)$ \\
\hline $\mathrm{C} 124-\mathrm{C} 125-\mathrm{C} 126-\mathrm{C} 127$ & $7(6)$ \\
\hline $\mathrm{N} 106-\mathrm{C} 126-\mathrm{C} 127-\mathrm{C} 128$ & $3(7)$ \\
\hline $\mathrm{C} 125-\mathrm{C} 126-\mathrm{C} 127-\mathrm{C} 128$ & $-180(4)$ \\
\hline $\mathrm{C} 128-\mathrm{C} 129-\mathrm{C} 130-\mathrm{N} 106$ & $0(6)$ \\
\hline $\mathrm{C} 128-\mathrm{C} 129-\mathrm{C} 130-\mathrm{C} 131$ & $-176(4)$ \\
\hline $\mathrm{N} 106-\mathrm{C} 130-\mathrm{C} 131-\mathrm{N} 107$ & $-5(4)$ \\
\hline $\mathrm{C} 129-\mathrm{C} 130-\mathrm{C} 131-\mathrm{N} 107$ & $171(5)$ \\
\hline $\mathrm{N} 106-\mathrm{C} 130-\mathrm{C} 131-\mathrm{C} 132$ & $171(5)$ \\
\hline $\mathrm{C} 129-\mathrm{C} 130-\mathrm{C} 131-\mathrm{C} 132$ & $-13(5)$ \\
\hline $\mathrm{N} 107-\mathrm{C} 131-\mathrm{C} 132-\mathrm{C} 133$ & $1(7)$ \\
\hline $\mathrm{C} 130-\mathrm{C} 131-\mathrm{C} 132-\mathrm{C} 133$ & $-175(6)$ \\
\hline $\mathrm{C} 131-\mathrm{C} 132-\mathrm{C} 133-\mathrm{C} 134$ & $-4(6)$ \\
\hline $\mathrm{C} 132-\mathrm{C} 133-\mathrm{C} 134-\mathrm{C} 135$ & $4(9)$ \\
\hline $\mathrm{C} 133-\mathrm{C} 134-\mathrm{C} 135-\mathrm{N} 107$ & $0(13)$ \\
\hline $\mathrm{C} 122-\mathrm{C} 121-\mathrm{N} 105-\mathrm{C} 125$ & $2(11)$ \\
\hline $\mathrm{C} 122-\mathrm{C} 121-\mathrm{N} 105-\mathrm{Mn} 1$ & $-177(6)$ \\
\hline $\mathrm{C} 124-\mathrm{C} 125-\mathrm{N} 105-\mathrm{C} 121$ & $-3(7)$ \\
\hline $\mathrm{C} 126-\mathrm{C} 125-\mathrm{N} 105-\mathrm{C} 121$ & $179(5)$ \\
\hline $\mathrm{C} 124-\mathrm{C} 125-\mathrm{N} 105-\mathrm{Mn} 1$ & $176(5)$ \\
\hline $\mathrm{C} 126-\mathrm{C} 125-\mathrm{N} 105-\mathrm{Mn} 1$ & $-2(5)$ \\
\hline $\mathrm{C} 129-\mathrm{C} 130-\mathrm{N} 106-\mathrm{C} 126$ & $2(5)$ \\
\hline $\mathrm{C} 131-\mathrm{C} 130-\mathrm{N} 106-\mathrm{C} 126$ & $178(4)$ \\
\hline $\mathrm{C} 129-\mathrm{C} 130-\mathrm{N} 106-\mathrm{Mn} 1$ & $-177(4)$ \\
\hline $\mathrm{C} 131-\mathrm{C} 130-\mathrm{N} 106-\mathrm{Mn} 1$ & $-1(4)$ \\
\hline $\mathrm{C} 127-\mathrm{C} 126-\mathrm{N} 106-\mathrm{C} 130$ & $-3(6)$ \\
\hline $\mathrm{C} 125-\mathrm{C} 126-\mathrm{N} 106-\mathrm{C} 130$ & $180(3)$ \\
\hline $\mathrm{C} 127-\mathrm{C} 126-\mathrm{N} 106-\mathrm{Mn} 1$ & $176(4)$ \\
\hline
\end{tabular}




$\begin{array}{ll}\mathrm{C} 24-\mathrm{C} 25-\mathrm{C} 26-\mathrm{N} 6 & -178.7(9) \\ \mathrm{N} 5-\mathrm{C} 25-\mathrm{C} 26-\mathrm{C} 27 & 177.2(9) \\ \mathrm{C} 24-\mathrm{C} 25-\mathrm{C} 26-\mathrm{C} 27 & -0.7(12) \\ \mathrm{N} 6-\mathrm{C} 26-\mathrm{C} 27-\mathrm{C} 28 & 3.6(12) \\ \mathrm{C} 25-\mathrm{C} 26-\mathrm{C} 27-\mathrm{C} 28 & -174.3(7) \\ \mathrm{C} 28-\mathrm{C} 29-\mathrm{C} 30-\mathrm{N} 6 & 2.5(11) \\ \mathrm{C} 28-\mathrm{C} 29-\mathrm{C} 30-\mathrm{C} 31 & -178.7(7) \\ \mathrm{N} 6-\mathrm{C} 30-\mathrm{C} 31-\mathrm{N} 7 & 3.5(10) \\ \mathrm{C} 29-\mathrm{C} 30-\mathrm{C} 31-\mathrm{N} 7 & -175.4(9) \\ \mathrm{N} 6-\mathrm{C} 30-\mathrm{C} 31-\mathrm{C} 32 & -173.7(9) \\ \mathrm{C} 29-\mathrm{C} 30-\mathrm{C} 31-\mathrm{C} 32 & 7.4(13) \\ \mathrm{N} 7-\mathrm{C} 31-\mathrm{C} 32-\mathrm{C} 33 & 0.9(13) \\ \mathrm{C} 30-\mathrm{C} 31-\mathrm{C} 32-\mathrm{C} 33 & 177.9(9) \\ \mathrm{C} 31-\mathrm{C} 32-\mathrm{C} 33-\mathrm{C} 34 & -0.3(13) \\ \mathrm{C} 32-\mathrm{C} 33-\mathrm{C} 34-\mathrm{C} 35 & -0.2(16) \\ \mathrm{C} 33-\mathrm{C} 34-\mathrm{C} 35-\mathrm{N} 7 & 0(2) \\ \mathrm{C} 22-\mathrm{C} 21-\mathrm{N} 5-\mathrm{C} 25 & 0.4(17) \\ \mathrm{C} 22-\mathrm{C} 21-\mathrm{N} 5-\mathrm{Mn} 1 & -177.3(10) \\ \mathrm{C} 24-\mathrm{C} 25-\mathrm{N} 5-\mathrm{C} 21 & 0.0(14) \\ \mathrm{C} 26-\mathrm{C} 25-\mathrm{N} 5-\mathrm{C} 21 & -177.9(9) \\ \mathrm{C} 24-\mathrm{C} 25-\mathrm{N} 5-\mathrm{Mn} 1 & 177.9(8) \\ \mathrm{C} 26-\mathrm{C} 25-\mathrm{N} 5-\mathrm{Mn} 1 & -0.1(10) \\ \mathrm{C} 29-\mathrm{C} 30-\mathrm{N} 6-\mathrm{C} 26 & -2.3(12)\end{array}$

$\begin{array}{ll}\mathrm{C} 125-\mathrm{C} 126-\mathrm{N} 106-\mathrm{Mn} 1 & -1(5) \\ \mathrm{C} 134-\mathrm{C} 135-\mathrm{N} 107-\mathrm{C} 131 & -3(11) \\ \mathrm{C} 134-\mathrm{C} 135-\mathrm{N} 107-\mathrm{Mn} 1 & 165(9) \\ \mathrm{C} 132-\mathrm{C} 131-\mathrm{N} 107-\mathrm{C} 135 & 3(8) \\ \mathrm{C} 130-\mathrm{C} 131-\mathrm{N} 107-\mathrm{C} 135 & 179(6) \\ \mathrm{C} 132-\mathrm{C} 131-\mathrm{N} 107-\mathrm{Mn} 1 & -167(5) \\ \mathrm{C} 130-\mathrm{C} 131-\mathrm{N} 107-\mathrm{M} 11 & 9(5) \\ \mathrm{C} 126-\mathrm{C} 127-\mathrm{C} 128-\mathrm{C} 138 & 177(4) \\ \mathrm{C} 126-\mathrm{C} 127-\mathrm{C} 128-\mathrm{C} 129 & -1(7) \\ \mathrm{C} 130-\mathrm{C} 129-\mathrm{C} 128-\mathrm{C} 127 & 0(6) \\ \mathrm{C} 130-\mathrm{C} 129-\mathrm{C} 128-\mathrm{C} 138 & -179(4) \\ \mathrm{N} 108-\mathrm{C} 136-\mathrm{C} 137-\mathrm{C} 138 & 0.0 \\ \mathrm{C} 136-\mathrm{C} 137-\mathrm{C} 138-\mathrm{C} 139 & 0.0 \\ \mathrm{C} 136-\mathrm{C} 137-\mathrm{C} 138-\mathrm{C} 128 & 177(3) \\ \mathrm{C} 127-\mathrm{C} 128-\mathrm{C} 138-\mathrm{C} 139 & -39(5) \\ \mathrm{C} 129-\mathrm{C} 128-\mathrm{C} 138-\mathrm{C} 139 & 140(4) \\ \mathrm{C} 127-\mathrm{C} 128-\mathrm{C} 138-\mathrm{C} 137 & 144(4) \\ \mathrm{C} 129-\mathrm{C} 128-\mathrm{C} 138-\mathrm{C} 137 & -37(5) \\ \mathrm{C} 137-\mathrm{C} 138-\mathrm{C} 139-\mathrm{C} 140 & 0.0 \\ \mathrm{C} 128-\mathrm{C} 138-\mathrm{C} 139-\mathrm{C} 140 & -177(3) \\ \mathrm{C} 138-\mathrm{C} 139-\mathrm{C} 140-\mathrm{N} 108 & 0.0 \\ \mathrm{C} 139-\mathrm{C} 140-\mathrm{N} 108-\mathrm{C} 136 & 0.0 \\ \mathrm{C} 137-\mathrm{C} 136-\mathrm{N} 108-\mathrm{C} 140 & 0.0 \\ & \end{array}$

Hydrogen-bond geometry $\left(\AA,{ }^{\circ}\right)$

\begin{tabular}{lllll}
\hline$D-\mathrm{H} \cdots A$ & $D-\mathrm{H}$ & $\mathrm{H} \cdots A$ & $D \cdots A$ & $D-\mathrm{H} \cdots A$ \\
\hline $\mathrm{C} 12-\mathrm{H} 12 \cdots \mathrm{F} 5 A^{\mathrm{i}}$ & 0.95 & 2.50 & $3.422(9)$ & 164 \\
$\mathrm{C} 15-\mathrm{H} 15 \cdots \mathrm{F} 12 A^{\mathrm{ii}}$ & 0.95 & 2.46 & $3.305(9)$ & 149 \\
$\mathrm{C} 16-\mathrm{H} 16 \cdots \mathrm{F} 7 A^{\mathrm{iii}}$ & 0.95 & 2.38 & $3.289(9)$ & 160 \\
$\mathrm{C} 19-\mathrm{H} 19 \cdots \mathrm{F} 5 A^{\mathrm{i}}$ & 0.95 & 2.41 & $3.328(10)$ & 162 \\
$\mathrm{C} 29-\mathrm{H} 29 \cdots \mathrm{N} 4^{\mathrm{iv}}$ & 0.95 & 2.35 & $3.270(8)$ & 163 \\
\hline
\end{tabular}

Symmetry codes: (i) $-x+1,-y+1,-z$; (ii) $x,-y+1 / 2, z-1 / 2$; (iii) $x+1, y, z$; (iv) $x-1, y, z$.

(2) Bis[4'-(pyridin-4-yl)-2,2':6',2"'-terpyridine]manganese(II) bis(hexafluoridophosphate) acetone monosolvate

Crystal data

$\left[\mathrm{Mn}\left(\mathrm{C}_{20} \mathrm{H}_{14} \mathrm{~N}_{4}\right)_{2}\right]\left(\mathrm{PF}_{6}\right)_{2} \cdot \mathrm{C}_{3} \mathrm{H}_{6} \mathrm{O}$

$M_{r}=1023.66$

Orthorhombic, $C 222_{1}$

$a=18.0996(15) \AA$

$b=27.470(2) \AA$

$c=8.5734(6) \AA$

$V=4262.7(6) \AA^{3}$

$Z=4$

$F(000)=2076$
$D_{\mathrm{x}}=1.595 \mathrm{Mg} \mathrm{m}^{-3}$

Mo $K \alpha$ radiation, $\lambda=0.71073 \AA$

Cell parameters from 6510 reflections

$\theta=2.7-25.2^{\circ}$

$\mu=0.48 \mathrm{~mm}^{-1}$

$T=150 \mathrm{~K}$

Block, yellow

$0.16 \times 0.08 \times 0.04 \mathrm{~mm}$ 


\section{Data collection}

Bruker APEXII CCD diffractometer

Radiation source: sealed tube

Graphite monochromator

$\varphi$ and $\omega$ scans

Absorption correction: multi-scan

(SADABS; Bruker, 2007)

$T_{\min }=0.955, T_{\max }=0.981$

\section{Refinement}

Refinement on $F^{2}$

Least-squares matrix: full

$R\left[F^{2}>2 \sigma\left(F^{2}\right)\right]=0.104$

$w R\left(F^{2}\right)=0.270$

$S=1.07$

3908 reflections

292 parameters

102 restraints

Hydrogen site location: inferred from

neighbouring sites
18921 measured reflections

3908 independent reflections

2683 reflections with $I>2 \sigma(I)$

$R_{\text {int }}=0.091$

$\theta_{\max }=25.4^{\circ}, \theta_{\min }=2.3^{\circ}$

$h=-21 \rightarrow 21$

$k=-31 \rightarrow 33$

$l=-10 \rightarrow 10$

$\mathrm{H}$-atom parameters constrained

$w=1 /\left[\sigma^{2}\left(F_{\mathrm{o}}^{2}\right)+(0.0919 P)^{2}+48.9339 P\right]$

where $P=\left(F_{\mathrm{o}}{ }^{2}+2 F_{\mathrm{c}}{ }^{2}\right) / 3$

$(\Delta / \sigma)_{\max }<0.001$

$\Delta \rho_{\max }=0.71$ e $\AA^{-3}$

$\Delta \rho_{\min }=-1.05$ e $\AA^{-3}$

Absolute structure: Flack $x$ determined using 836 quotients $[(\mathrm{I}+)-(\mathrm{I}-)] /[(\mathrm{I}+)+(\mathrm{I}-)]$ (Parsons et al. (2013)

Absolute structure parameter: 0.21 (2)

Special details

Geometry. All e.s.d.'s (except the e.s.d. in the dihedral angle between two 1.s. planes) are estimated using the full covariance matrix. The cell e.s.d.'s are taken into account individually in the estimation of e.s.d.'s in distances, angles and torsion angles; correlations between e.s.d.'s in cell parameters are only used when they are defined by crystal symmetry. An approximate (isotropic) treatment of cell e.s.d.'s is used for estimating e.s.d.'s involving 1.s. planes.

Fractional atomic coordinates and isotropic or equivalent isotropic displacement parameters $\left(\AA^{2}\right)$

\begin{tabular}{llllll}
\hline & $x$ & $y$ & $z$ & $U_{\text {iso }} * / U_{\text {eq }}$ & Occ. $(<1)$ \\
\hline Mn1 & 0.5000 & $0.77635(9)$ & 0.7500 & $0.0370(7)$ & \\
C1 & $0.4292(7)$ & $0.7832(5)$ & $1.0872(14)$ & $0.048(3)$ & \\
H1 & 0.4307 & 0.8172 & 1.0665 & $0.058^{*}$ & \\
C2 & $0.4020(7)$ & $0.7676(6)$ & $1.2295(16)$ & $0.054(4)$ & $0.065^{*}$ \\
H2 & 0.3866 & 0.7902 & 1.3069 & $0.048(3)$ & \\
C3 & $0.3980(7)$ & $0.7181(5)$ & $1.2545(16)$ & $0.058^{*}$ & \\
H3 & 0.3784 & 0.7062 & 1.3500 & $0.043(3)$ & \\
C4 & $0.4219(7)$ & $0.6858(5)$ & $1.1433(14)$ & $0.051^{*}$ & \\
H4 & 0.4197 & 0.6517 & 1.1621 & $0.039(3)$ & \\
C5 & $0.4488(7)$ & $0.7032(5)$ & $1.0052(13)$ & $0.032(3)$ & \\
C6 & $0.4751(6)$ & $0.6721(4)$ & $0.8756(12)$ & $0.048(3)$ & \\
C7 & $0.4750(7)$ & $0.6216(5)$ & $0.8755(15)$ & $0.058^{*}$ & \\
H7 & 0.4573 & 0.6046 & 0.9644 & $0.044(4)$ & \\
C8 & 0.5000 & $0.5958(6)$ & 0.7500 & $0.050(4)$ & \\
C12 & $0.3343(7)$ & $0.7696(6)$ & $0.6153(14)$ & $0.060^{*}$ & \\
H12 & 0.3387 & 0.7357 & 0.6366 & $0.056(4)$ & \\
C13 & $0.2723(8)$ & $0.7871(6)$ & $0.5405(16)$ & $0.068^{*}$ & \\
H13 & 0.2342 & 0.7651 & 0.5120 & $0.061(4)$ \\
C14 & $0.2645(8)$ & $0.8344(7)$ & $0.5069(18)$ & 0.4539 & \\
H14 & 0.2217 & 0.8457 & & &
\end{tabular}




\begin{tabular}{|c|c|c|c|c|c|}
\hline $\mathrm{C} 15$ & $0.3211(7)$ & $0.8675(6)$ & $0.5515(17)$ & $0.054(4)$ & \\
\hline H15 & 0.3180 & 0.9012 & 0.5279 & $0.064 *$ & \\
\hline $\mathrm{C} 16$ & $0.3811(7)$ & $0.8483(5)$ & $0.6310(14)$ & $0.040(3)$ & \\
\hline $\mathrm{C} 17$ & $0.4424(7)$ & $0.8814(5)$ & $0.6884(13)$ & 0.039 & \\
\hline $\mathrm{C} 18$ & $0.4412(7)$ & $0.9303(5)$ & $0.6864(14)$ & $0.041(3)$ & \\
\hline H18 & 0.4002 & 0.9468 & 0.6417 & $0.049^{*}$ & \\
\hline C19 & 0.5000 & $0.9574(6)$ & 0.7500 & $0.046(4)$ & \\
\hline $\mathrm{C} 20$ & 0.5000 & $1.0098(7)$ & 0.7500 & $0.054(5)$ & \\
\hline $\mathrm{C} 21$ & $0.4345(8)$ & $1.0377(5)$ & $0.7816(19)$ & $0.058(4)$ & \\
\hline $\mathrm{H} 21$ & 0.3892 & 1.0217 & 0.8040 & $0.069^{*}$ & \\
\hline $\mathrm{C} 22$ & $0.4377(9)$ & $1.0875(5)$ & $0.779(2)$ & $0.071(5)$ & \\
\hline $\mathrm{H} 22$ & 0.3935 & 1.1051 & 0.7990 & $0.085^{*}$ & \\
\hline N1 & $0.4541(6)$ & $0.7520(4)$ & $0.9758(11)$ & 0.039 & \\
\hline N2 & 0.5000 & $0.6970(5)$ & 0.7500 & 0.035 & \\
\hline N4 & 0.5000 & $0.8560(5)$ & 0.7500 & $0.041(3)$ & \\
\hline N5 & $0.3898(6)$ & $0.8012(4)$ & $0.6593(11)$ & $0.039(2)$ & \\
\hline N6 & 0.5000 & $1.1129(5)$ & 0.7500 & $0.070(5)$ & \\
\hline C109 & 0.5000 & $0.5411(6)$ & 0.7500 & 0.049 (4) & 0.5 \\
\hline $\mathrm{C} 110$ & $0.4556(18)$ & $0.5139(11)$ & $0.646(4)$ & $0.058(6)$ & 0.5 \\
\hline H110 & 0.4251 & 0.5288 & 0.5695 & $0.070^{*}$ & 0.5 \\
\hline C111 & $0.460(2)$ & $0.4652(12)$ & $0.663(4)$ & $0.061(6)$ & 0.5 \\
\hline H111 & 0.4270 & 0.4475 & 0.5977 & $0.074 *$ & 0.5 \\
\hline N13 & 0.5000 & $0.4386(6)$ & 0.7500 & $0.080(6)$ & 0.5 \\
\hline C209 & 0.5000 & $0.5411(6)$ & 0.7500 & $0.049(4)$ & 0.5 \\
\hline $\mathrm{C} 210$ & $0.4316(18)$ & $0.5177(10)$ & $0.765(4)$ & $0.058(6)$ & 0.5 \\
\hline $\mathrm{H} 210$ & 0.3863 & 0.5347 & 0.7785 & $0.070^{*}$ & 0.5 \\
\hline $\mathrm{C} 211$ & $0.436(2)$ & $0.4708(10)$ & $0.758(4)$ & $0.061(6)$ & 0.5 \\
\hline $\mathrm{H} 211$ & 0.3894 & 0.4545 & 0.7588 & $0.074 *$ & 0.5 \\
\hline $\mathrm{N} 23$ & 0.5000 & $0.4386(6)$ & 0.7500 & $0.080(6)$ & 0.5 \\
\hline P1 & $0.7697(4)$ & $0.8833(3)$ & $0.4862(10)$ & $0.0683(16)^{*}$ & 0.4 \\
\hline F11 & $0.7203(7)$ & 0.8354 (4) & $0.4710(17)$ & $0.0978(19)^{*}$ & 0.4 \\
\hline F12 & $0.7479(8)$ & $0.8984(5)$ & $0.3127(13)$ & $0.0978(19)^{*}$ & 0.4 \\
\hline F13 & $0.8190(7)$ & $0.9311(4)$ & $0.5015(17)$ & $0.0978(19)^{*}$ & 0.4 \\
\hline F14 & $0.7915(8)$ & $0.8681(5)$ & $0.6597(13)$ & $0.0978(19)^{*}$ & 0.4 \\
\hline F15 & $0.8393(7)$ & $0.8541(5)$ & $0.4211(17)$ & $0.0978(19)^{*}$ & 0.4 \\
\hline F16 & $0.7000(7)$ & $0.9124(5)$ & $0.5513(17)$ & $0.0978(19)^{*}$ & 0.4 \\
\hline $\mathrm{P} 2$ & $0.7698(5)$ & $0.8722(3)$ & $0.4736(11)$ & $0.0683(16)^{*}$ & 0.6 \\
\hline F21 & $0.7250(10)$ & $0.8513(7)$ & $0.330(2)$ & $0.0978(19)^{*}$ & 0.6 \\
\hline F22 & $0.7976(10)$ & $0.9225(7)$ & $0.422(2)$ & $0.0978(19)^{*}$ & 0.6 \\
\hline F23 & $0.8155(10)$ & $0.8818(7)$ & $0.635(2)$ & $0.0978(19)^{*}$ & 0.6 \\
\hline F24 & $0.7524(10)$ & $0.8204(7)$ & $0.557(2)$ & $0.0978(19)^{*}$ & 0.6 \\
\hline F25 & $0.8441(10)$ & $0.8478(7)$ & $0.396(2)$ & $0.0978(19)^{*}$ & 0.6 \\
\hline F26 & $0.6985(10)$ & $0.8939(7)$ & $0.548(2)$ & $0.0978(19)^{*}$ & 0.6 \\
\hline O90 & $0.8075(9)$ & $0.5064(8)$ & $0.520(3)$ & $0.066(6)^{*}$ & 0.5 \\
\hline C91 & $0.746(2)$ & $0.4862(10)$ & $0.754(3)$ & $0.126(10)^{*}$ & 0.5 \\
\hline H91A & 0.7806 & 0.4587 & 0.7596 & $0.188^{*}$ & 0.5 \\
\hline H91B & 0.6956 & 0.4745 & 0.7697 & $0.188^{*}$ & 0.5 \\
\hline H91C & 0.7586 & 0.5100 & 0.8350 & $0.188^{*}$ & 0.5 \\
\hline
\end{tabular}




\begin{tabular}{llllll} 
C90 & $0.7521(10)$ & $0.5095(4)$ & $0.599(2)$ & $0.126(10)^{*}$ & 0.5 \\
C92 & $0.6893(17)$ & $0.5377(10)$ & $0.534(5)$ & $0.126(10)^{*}$ & 0.5 \\
H92A & 0.6432 & 0.5200 & 0.5533 & $0.188^{*}$ & 0.5 \\
H92B & 0.6962 & 0.5421 & 0.4218 & $0.188^{*}$ & 0.5 \\
H92C & 0.6870 & 0.5696 & 0.5853 & $0.188^{*}$ & 0.5 \\
\hline
\end{tabular}

Atomic displacement parameters $\left(\AA^{2}\right)$

\begin{tabular}{|c|c|c|c|c|c|c|}
\hline & $U^{11}$ & $U^{22}$ & $U^{33}$ & $U^{12}$ & $U^{13}$ & $U^{23}$ \\
\hline Mn1 & $0.0534(15)$ & $0.0331(13)$ & $0.0246(11)$ & 0.000 & $0.0046(13)$ & 0.000 \\
\hline $\mathrm{C} 1$ & $0.055(8)$ & $0.054(9)$ & $0.036(6)$ & $-0.007(7)$ & $0.017(6)$ & $-0.010(6)$ \\
\hline $\mathrm{C} 2$ & $0.051(8)$ & $0.072(10)$ & $0.039(8)$ & $-0.006(7)$ & $0.007(6)$ & $-0.010(8)$ \\
\hline $\mathrm{C} 3$ & $0.052(7)$ & $0.066(9)$ & $0.028(5)$ & $-0.003(6)$ & $0.008(6)$ & $-0.006(9)$ \\
\hline $\mathrm{C} 4$ & $0.053(8)$ & $0.048(8)$ & $0.026(6)$ & $-0.004(6)$ & $0.001(6)$ & $0.003(6)$ \\
\hline $\mathrm{C} 5$ & $0.046(7)$ & $0.047(8)$ & $0.024(6)$ & $0.002(6)$ & $0.001(5)$ & $-0.003(5)$ \\
\hline C6 & $0.039(7)$ & $0.038(7)$ & $0.020(5)$ & $0.001(5)$ & $0.001(5)$ & $0.001(5)$ \\
\hline $\mathrm{C} 7$ & $0.059(9)$ & $0.052(8)$ & $0.034(6)$ & $-0.009(6)$ & $0.000(6)$ & $0.009(6)$ \\
\hline $\mathrm{C} 8$ & $0.055(11)$ & $0.032(9)$ & $0.044(10)$ & 0.000 & $-0.011(12)$ & 0.000 \\
\hline $\mathrm{C} 12$ & $0.036(7)$ & $0.082(11)$ & $0.033(6)$ & $-0.013(7)$ & $0.004(6)$ & $0.004(7)$ \\
\hline $\mathrm{C} 13$ & $0.040(7)$ & $0.083(8)$ & $0.046(8)$ & $-0.017(8)$ & $0.006(6)$ & $-0.010(7)$ \\
\hline C14 & $0.035(7)$ & $0.091(9)$ & $0.056(9)$ & $-0.004(8)$ & $0.008(7)$ & $0.004(8)$ \\
\hline $\mathrm{C} 15$ & $0.029(7)$ & $0.080(11)$ & $0.053(8)$ & $-0.002(7)$ & $-0.001(6)$ & $0.011(8)$ \\
\hline $\mathrm{C} 16$ & $0.033(6)$ & $0.050(8)$ & $0.036(6)$ & $-0.007(6)$ & $0.002(5)$ & $-0.003(6)$ \\
\hline $\mathrm{C} 17$ & $0.046(7)$ & $0.042(8)$ & $0.027(5)$ & $-0.006(6)$ & $0.004(5)$ & $-0.008(5)$ \\
\hline $\mathrm{C} 18$ & $0.039(7)$ & $0.046(8)$ & $0.039(7)$ & $0.005(6)$ & $0.004(6)$ & $0.002(5)$ \\
\hline C19 & $0.042(10)$ & $0.035(9)$ & 0.060 & 0.000 & $-0.003(12)$ & 0.000 \\
\hline $\mathrm{C} 20$ & $0.045(10)$ & $0.057(12)$ & 0.059 (12) & 0.000 & $0.012(13)$ & 0.000 \\
\hline $\mathrm{C} 21$ & $0.053(9)$ & $0.054(9)$ & $0.066(11)$ & $0.008(7)$ & $0.005(8)$ & $0.003(8)$ \\
\hline $\mathrm{C} 22$ & $0.065(10)$ & $0.044(8)$ & 0.105 (16) & $0.012(7)$ & $0.011(10)$ & $0.002(9)$ \\
\hline N1 & $0.057(7)$ & $0.039(6)$ & $0.022(5)$ & $-0.002(5)$ & $0.002(5)$ & $-0.004(4)$ \\
\hline $\mathrm{N} 2$ & $0.051(8)$ & $0.035(7)$ & $0.020(6)$ & 0.000 & $-0.005(8)$ & 0.000 \\
\hline N4 & $0.049(8)$ & $0.047(8)$ & $0.027(7)$ & 0.000 & $-0.010(8)$ & 0.000 \\
\hline N5 & $0.047(6)$ & $0.038(6)$ & $0.030(5)$ & $-0.007(5)$ & $0.007(5)$ & $0.001(4)$ \\
\hline N6 & $0.061(11)$ & $0.032(9)$ & $0.116(15)$ & 0.000 & 0.007 (14) & 0.000 \\
\hline C109 & $0.075(13)$ & $0.029(9)$ & $0.042(9)$ & 0.000 & $-0.003(13)$ & 0.000 \\
\hline $\mathrm{C} 110$ & $0.069(15)$ & $0.046(12)$ & $0.060(14)$ & $0.004(10)$ & $-0.017(13)$ & $-0.012(12)$ \\
\hline C111 & $0.086(18)$ & $0.046(12)$ & $0.052(16)$ & $-0.001(11)$ & $0.002(14)$ & $-0.005(14)$ \\
\hline N13 & $0.132(18)$ & $0.034(10)$ & $0.075(12)$ & 0.000 & $-0.027(16)$ & 0.000 \\
\hline C209 & 0.075 (13) & $0.029(9)$ & $0.042(9)$ & 0.000 & $-0.003(13)$ & 0.000 \\
\hline $\mathrm{C} 210$ & $0.069(15)$ & $0.046(12)$ & $0.060(14)$ & $0.004(10)$ & $-0.017(13)$ & $-0.012(12)$ \\
\hline $\mathrm{C} 211$ & $0.086(18)$ & $0.046(12)$ & $0.052(16)$ & $-0.001(11)$ & $0.002(14)$ & $-0.005(14)$ \\
\hline N23 & $0.132(18)$ & $0.034(10)$ & $0.075(12)$ & 0.000 & $-0.027(16)$ & 0.000 \\
\hline
\end{tabular}

Geometric parameters $\left(\AA,{ }^{\circ}\right)$

\begin{tabular}{llll}
\hline $\mathrm{Mn} 1-\mathrm{N} 1$ & $2.210(10)$ & $\mathrm{C} 21-\mathrm{C} 22$ & $1.37(2)$ \\
$\mathrm{Mn} 1-\mathrm{N} 2$ & $2.180(13)$ & $\mathrm{C} 21-\mathrm{H} 21$ & 0.9500 \\
$\mathrm{Mn} 1-\mathrm{N} 4$ & $2.187(14)$ & $\mathrm{C} 22-\mathrm{N} 6$ & $1.351(18)$
\end{tabular}




\begin{tabular}{|c|c|}
\hline Mn1-N5 & $2.247(11)$ \\
\hline $\mathrm{Mn} 1-\mathrm{N} 1^{\mathrm{i}}$ & $2.210(10)$ \\
\hline $\mathrm{Mn} 1-\mathrm{N} 5^{\mathrm{i}}$ & $2.247(11)$ \\
\hline $\mathrm{C} 1-\mathrm{N} 1$ & $1.358(16)$ \\
\hline $\mathrm{C} 1-\mathrm{C} 2$ & $1.384(18)$ \\
\hline $\mathrm{C} 1-\mathrm{H} 1$ & 0.9500 \\
\hline $\mathrm{C} 2-\mathrm{C} 3$ & $1.379(19)$ \\
\hline $\mathrm{C} 2-\mathrm{H} 2$ & 0.9500 \\
\hline $\mathrm{C} 3-\mathrm{C} 4$ & $1.372(18)$ \\
\hline $\mathrm{C} 3-\mathrm{H} 3$ & 0.9500 \\
\hline $\mathrm{C} 4-\mathrm{C} 5$ & $1.366(16)$ \\
\hline $\mathrm{C} 4-\mathrm{H} 4$ & 0.9500 \\
\hline $\mathrm{C} 5-\mathrm{N} 1$ & $1.370(16)$ \\
\hline $\mathrm{C} 5-\mathrm{C} 6$ & $1.480(15)$ \\
\hline $\mathrm{C} 6-\mathrm{N} 2$ & $1.353(13)$ \\
\hline $\mathrm{C} 6-\mathrm{C} 7$ & $1.387(18)$ \\
\hline $\mathrm{C} 7-\mathrm{C} 8$ & $1.364(16)$ \\
\hline $\mathrm{C} 7-\mathrm{H} 7$ & 0.9500 \\
\hline $\mathrm{C} 8-\mathrm{C}^{\mathrm{i}}$ & $1.364(16)$ \\
\hline C8-C109 & $1.50(2)$ \\
\hline $\mathrm{C} 8-\mathrm{C} 209$ & $1.50(2)$ \\
\hline $\mathrm{C} 12-\mathrm{C} 13$ & $1.38(2)$ \\
\hline $\mathrm{C} 12-\mathrm{N} 5$ & $1.380(16)$ \\
\hline $\mathrm{C} 12-\mathrm{H} 12$ & 0.9500 \\
\hline $\mathrm{C} 13-\mathrm{C} 14$ & $1.34(2)$ \\
\hline $\mathrm{C} 13-\mathrm{H} 13$ & 0.9500 \\
\hline $\mathrm{C} 14-\mathrm{C} 15$ & $1.42(2)$ \\
\hline C14-H14 & 0.9500 \\
\hline $\mathrm{C} 15-\mathrm{C} 16$ & $1.386(18)$ \\
\hline C15-H15 & 0.9500 \\
\hline $\mathrm{C} 16-\mathrm{N} 5$ & $1.326(16)$ \\
\hline $\mathrm{C} 16-\mathrm{C} 17$ & $1.517(17)$ \\
\hline $\mathrm{C} 17-\mathrm{C} 18$ & $1.344(18)$ \\
\hline $\mathrm{C} 17-\mathrm{N} 4$ & $1.361(15)$ \\
\hline $\mathrm{C} 18-\mathrm{C} 19$ & $1.408(16)$ \\
\hline C18-H18 & 0.9500 \\
\hline $\mathrm{C} 19-\mathrm{C} 18^{\mathrm{i}}$ & $1.408(16)$ \\
\hline $\mathrm{C} 19-\mathrm{C} 20$ & $1.44(3)$ \\
\hline $\mathrm{C} 20-\mathrm{C} 21^{\mathrm{i}}$ & $1.437(17)$ \\
\hline $\mathrm{C} 20-\mathrm{C} 21$ & $1.437(17)$ \\
\hline $\mathrm{N} 1-\mathrm{Mn} 1-\mathrm{N} 1^{\mathrm{i}}$ & $144.8(5)$ \\
\hline $\mathrm{N} 1-\mathrm{Mn} 1-\mathrm{N} 2$ & $72.4(3)$ \\
\hline $\mathrm{N} 1-\mathrm{Mn} 1-\mathrm{N} 4$ & $107.6(3)$ \\
\hline $\mathrm{N} 1-\mathrm{Mn} 1-\mathrm{N} 5$ & $93.5(4)$ \\
\hline $\mathrm{N} 1-\mathrm{Mn} 1-\mathrm{N} 5^{\mathrm{i}}$ & $97.0(4)$ \\
\hline $\mathrm{N} 2-\mathrm{Mn} 1-\mathrm{N} 4$ & 180.0 \\
\hline $\mathrm{N} 2-\mathrm{Mn} 1-\mathrm{N} 5$ & $107.7(3)$ \\
\hline
\end{tabular}

\begin{tabular}{|c|c|}
\hline $\mathrm{C} 22-\mathrm{H} 22$ & 0.9500 \\
\hline $\mathrm{N} 2-\mathrm{C} 6^{\mathrm{i}}$ & $1.353(13)$ \\
\hline $\mathrm{N} 4-\mathrm{C} 17^{\mathrm{i}}$ & $1.361(15)$ \\
\hline $\mathrm{N} 6-\mathrm{C} 22^{\mathrm{i}}$ & $1.351(18)$ \\
\hline $\mathrm{C} 109-\mathrm{C} 110$ & $1.41(3)$ \\
\hline $\mathrm{C} 109-\mathrm{C} 110^{\mathrm{i}}$ & $1.41(3)$ \\
\hline $\mathrm{C} 110-\mathrm{C} 111$ & $1.35(4)$ \\
\hline $\mathrm{C} 110-\mathrm{H} 110$ & 0.9500 \\
\hline $\mathrm{C} 111-\mathrm{N} 13$ & $1.27(3)$ \\
\hline C111-H111 & 0.9500 \\
\hline $\mathrm{N} 13-\mathrm{C} 111^{\mathrm{i}}$ & $1.27(4)$ \\
\hline $\mathrm{C} 209-\mathrm{C} 210^{\mathrm{i}}$ & $1.40(3)$ \\
\hline $\mathrm{C} 209-\mathrm{C} 210$ & $1.40(3)$ \\
\hline $\mathrm{C} 210-\mathrm{C} 211$ & $1.29(4)$ \\
\hline $\mathrm{C} 210-\mathrm{H} 210$ & 0.9500 \\
\hline $\mathrm{C} 211-\mathrm{N} 23$ & $1.46(3)$ \\
\hline $\mathrm{C} 211-\mathrm{H} 211$ & 0.9500 \\
\hline $\mathrm{N} 23-\mathrm{C} 211^{\mathrm{i}}$ & $1.46(3)$ \\
\hline $\mathrm{P} 1-\mathrm{F} 14$ & $1.594(7)$ \\
\hline $\mathrm{P} 1-\mathrm{F} 16$ & $1.594(7)$ \\
\hline $\mathrm{P} 1-\mathrm{F} 11$ & $1.594(7)$ \\
\hline $\mathrm{P} 1-\mathrm{F} 13$ & $1.594(7)$ \\
\hline $\mathrm{P} 1-\mathrm{F} 12$ & $1.594(7)$ \\
\hline $\mathrm{P} 1-\mathrm{F} 15$ & $1.594(7)$ \\
\hline $\mathrm{P} 2-\mathrm{F} 22$ & $1.54(2)$ \\
\hline $\mathrm{P} 2-\mathrm{F} 26$ & 1.557 (19) \\
\hline $\mathrm{P} 2-\mathrm{F} 21$ & $1.578(19)$ \\
\hline $\mathrm{P} 2-\mathrm{F} 24$ & $1.62(2)$ \\
\hline $\mathrm{P} 2-\mathrm{F} 23$ & $1.634(19)$ \\
\hline $\mathrm{P} 2-\mathrm{F} 25$ & $1.644(19)$ \\
\hline $\mathrm{O} 90-\mathrm{C} 90$ & $1.209(4)$ \\
\hline C91-C90 & $1.481(4)$ \\
\hline C91-H91A & 0.9800 \\
\hline C91-H91B & 0.9800 \\
\hline C91-H91C & 0.9800 \\
\hline $\mathrm{C} 90-\mathrm{C} 92$ & $1.482(4)$ \\
\hline $\mathrm{C} 92-\mathrm{H} 92 \mathrm{~A}$ & 0.9800 \\
\hline C92-H92B & 0.9800 \\
\hline $\mathrm{C} 92-\mathrm{H} 92 \mathrm{C}$ & 0.9800 \\
\hline $\mathrm{C} 66^{\mathrm{i}-\mathrm{N} 2}-\mathrm{C} 6$ & $119.3(14)$ \\
\hline $\mathrm{C} 6{ }^{\mathrm{i}}-\mathrm{N} 2-\mathrm{Mn} 1$ & $120.4(7)$ \\
\hline $\mathrm{C} 6-\mathrm{N} 2-\mathrm{Mn} 1$ & $120.4(7)$ \\
\hline $\mathrm{C} 17^{\mathrm{i}}-\mathrm{N} 4-\mathrm{C} 17$ & $118.3(15)$ \\
\hline $\mathrm{C} 17^{\mathrm{i}}-\mathrm{N} 4-\mathrm{Mn} 1$ & $120.9(7)$ \\
\hline $\mathrm{C} 17-\mathrm{N} 4-\mathrm{Mn} 1$ & $120.9(7)$ \\
\hline $\mathrm{C} 16-\mathrm{N} 5-\mathrm{C} 12$ & $118.6(12)$ \\
\hline
\end{tabular}




\begin{tabular}{|c|c|c|c|}
\hline $\mathrm{N} 4-\mathrm{Mn} 1-\mathrm{N} 5$ & $72.3(3)$ & $\mathrm{C} 16-\mathrm{N} 5-\mathrm{Mn} 1$ & $117.6(8)$ \\
\hline $\mathrm{N} 5-\mathrm{Mn} 1-\mathrm{N} 5^{\mathrm{i}}$ & $144.7(5)$ & $\mathrm{C} 12-\mathrm{N} 5-\mathrm{Mn} 1$ & $123.4(9)$ \\
\hline $\mathrm{N} 2-\mathrm{Mn} 1-\mathrm{N} 1^{\mathrm{i}}$ & $72.4(3)$ & $\mathrm{C} 22-\mathrm{N} 6-\mathrm{C} 22^{\mathrm{i}}$ & $117.6(17)$ \\
\hline $\mathrm{N} 4-\mathrm{Mn} 1-\mathrm{N} 1^{\mathrm{i}}$ & $107.6(3)$ & $\mathrm{C} 110-\mathrm{C} 109-\mathrm{C} 110^{\mathrm{i}}$ & $116(3)$ \\
\hline $\mathrm{N} 2-\mathrm{Mn} 1-\mathrm{N} 5^{\mathrm{i}}$ & $107.7(3)$ & $\mathrm{C} 110-\mathrm{C} 109-\mathrm{C} 8$ & $121.9(14)$ \\
\hline $\mathrm{N} 4-\mathrm{Mn} 1-\mathrm{N} 5^{\mathrm{i}}$ & $72.3(3)$ & $\mathrm{C} 110-\mathrm{C} 109-\mathrm{C} 8$ & $121.9(14)$ \\
\hline $\mathrm{N} 1^{\mathrm{i}}-\mathrm{Mn} 1-\mathrm{N} 5^{\mathrm{i}}$ & $93.5(4)$ & $\mathrm{C} 111-\mathrm{C} 110-\mathrm{C} 109$ & $115(3)$ \\
\hline $\mathrm{N} 1-\mathrm{C} 1-\mathrm{C} 2$ & $122.8(14)$ & $\mathrm{C} 111-\mathrm{C} 110-\mathrm{H} 110$ & 122.5 \\
\hline $\mathrm{N} 1-\mathrm{C} 1-\mathrm{H} 1$ & 118.6 & $\mathrm{C} 109-\mathrm{C} 110-\mathrm{H} 110$ & 122.5 \\
\hline $\mathrm{C} 2-\mathrm{C} 1-\mathrm{H} 1$ & 118.6 & $\mathrm{~N} 13-\mathrm{C} 111-\mathrm{C} 110$ & $132(3)$ \\
\hline $\mathrm{C} 3-\mathrm{C} 2-\mathrm{C} 1$ & $117.5(14)$ & N13-C111-H111 & 114.1 \\
\hline $\mathrm{C} 3-\mathrm{C} 2-\mathrm{H} 2$ & 121.3 & $\mathrm{C} 110-\mathrm{C} 111-\mathrm{H} 111$ & 114.1 \\
\hline $\mathrm{C} 1-\mathrm{C} 2-\mathrm{H} 2$ & 121.3 & $\mathrm{C} 111-\mathrm{N} 13-\mathrm{C} 111^{\mathrm{i}}$ & $110(3)$ \\
\hline $\mathrm{C} 4-\mathrm{C} 3-\mathrm{C} 2$ & $120.9(14)$ & $\mathrm{C} 210^{\mathrm{i}}-\mathrm{C} 209-\mathrm{C} 210$ & $125(3)$ \\
\hline $\mathrm{C} 4-\mathrm{C} 3-\mathrm{H} 3$ & 119.6 & $\mathrm{C} 210-\mathrm{C} 209-\mathrm{C} 8$ & $117.3(13)$ \\
\hline $\mathrm{C} 2-\mathrm{C} 3-\mathrm{H} 3$ & 119.6 & $\mathrm{C} 210-\mathrm{C} 209-\mathrm{C} 8$ & $117.3(13)$ \\
\hline $\mathrm{C} 5-\mathrm{C} 4-\mathrm{C} 3$ & $119.2(13)$ & $\mathrm{C} 211-\mathrm{C} 210-\mathrm{C} 209$ & $114(3)$ \\
\hline $\mathrm{C} 5-\mathrm{C} 4-\mathrm{H} 4$ & 120.4 & $\mathrm{C} 211-\mathrm{C} 210-\mathrm{H} 210$ & 123.1 \\
\hline $\mathrm{C} 3-\mathrm{C} 4-\mathrm{H} 4$ & 120.4 & $\mathrm{C} 209-\mathrm{C} 210-\mathrm{H} 210$ & 123.1 \\
\hline $\mathrm{C} 4-\mathrm{C} 5-\mathrm{N} 1$ & $121.8(12)$ & $\mathrm{C} 210-\mathrm{C} 211-\mathrm{N} 23$ & $131(3)$ \\
\hline $\mathrm{C} 4-\mathrm{C} 5-\mathrm{C} 6$ & $124.3(12)$ & $\mathrm{C} 210-\mathrm{C} 211-\mathrm{H} 211$ & 114.7 \\
\hline $\mathrm{N} 1-\mathrm{C} 5-\mathrm{C} 6$ & $113.9(11)$ & $\mathrm{N} 23-\mathrm{C} 211-\mathrm{H} 211$ & 114.7 \\
\hline $\mathrm{N} 2-\mathrm{C} 6-\mathrm{C} 7$ & $120.3(11)$ & 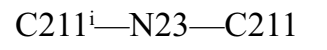 & $106(3)$ \\
\hline $\mathrm{N} 2-\mathrm{C} 6-\mathrm{C} 5$ & $114.4(11)$ & $\mathrm{F} 14-\mathrm{P} 1-\mathrm{F} 16$ & $90.00(6)$ \\
\hline $\mathrm{C} 7-\mathrm{C} 6-\mathrm{C} 5$ & $125.2(10)$ & $\mathrm{F} 14-\mathrm{P} 1-\mathrm{F} 11$ & $90.00(6)$ \\
\hline $\mathrm{C} 8-\mathrm{C} 7-\mathrm{C} 6$ & $121.3(12)$ & $\mathrm{F} 16-\mathrm{P} 1-\mathrm{F} 11$ & $90.00(6)$ \\
\hline $\mathrm{C} 8-\mathrm{C} 7-\mathrm{H} 7$ & 119.4 & $\mathrm{~F} 14-\mathrm{P} 1-\mathrm{F} 13$ & $90.00(6)$ \\
\hline $\mathrm{C} 6-\mathrm{C} 7-\mathrm{H} 7$ & 119.4 & $\mathrm{~F} 16-\mathrm{P} 1-\mathrm{F} 13$ & $90.00(6)$ \\
\hline $\mathrm{C} 7^{\mathrm{i}}-\mathrm{C} 8-\mathrm{C} 7$ & $117.5(16)$ & $\mathrm{F} 11-\mathrm{P} 1-\mathrm{F} 13$ & $180.00(10)$ \\
\hline $\mathrm{C} 7-\mathrm{C} 8-\mathrm{C} 109$ & $121.2(8)$ & $\mathrm{F} 14-\mathrm{P} 1-\mathrm{F} 12$ & $180.0(3)$ \\
\hline $\mathrm{C} 7-\mathrm{C} 8-\mathrm{C} 109$ & $121.2(8)$ & $\mathrm{F} 16-\mathrm{P} 1-\mathrm{F} 12$ & $90.00(6)$ \\
\hline $\mathrm{C} 7-\mathrm{C} 8-\mathrm{C} 209$ & $121.2(8)$ & $\mathrm{F} 11-\mathrm{P} 1-\mathrm{F} 12$ & $90.00(6)$ \\
\hline $\mathrm{C} 7-\mathrm{C} 8-\mathrm{C} 209$ & $121.2(8)$ & $\mathrm{F} 13-\mathrm{P} 1-\mathrm{F} 12$ & $90.00(6)$ \\
\hline $\mathrm{C} 13-\mathrm{C} 12-\mathrm{N} 5$ & $119.9(14)$ & $\mathrm{F} 14-\mathrm{P} 1-\mathrm{F} 15$ & $90.00(6)$ \\
\hline $\mathrm{C} 13-\mathrm{C} 12-\mathrm{H} 12$ & 120.1 & $\mathrm{~F} 16-\mathrm{P} 1-\mathrm{F} 15$ & $180.0(10)$ \\
\hline $\mathrm{N} 5-\mathrm{C} 12-\mathrm{H} 12$ & 120.1 & $\mathrm{~F} 11-\mathrm{P} 1-\mathrm{F} 15$ & $90.00(6)$ \\
\hline $\mathrm{C} 14-\mathrm{C} 13-\mathrm{C} 12$ & $121.8(14)$ & $\mathrm{F} 13-\mathrm{P} 1-\mathrm{F} 15$ & $90.00(6)$ \\
\hline $\mathrm{C} 14-\mathrm{C} 13-\mathrm{H} 13$ & 119.1 & $\mathrm{~F} 12-\mathrm{P} 1-\mathrm{F} 15$ & $90.00(6)$ \\
\hline $\mathrm{C} 12-\mathrm{C} 13-\mathrm{H} 13$ & 119.1 & $\mathrm{~F} 22-\mathrm{P} 2-\mathrm{F} 26$ & $92.5(11)$ \\
\hline $\mathrm{C} 13-\mathrm{C} 14-\mathrm{C} 15$ & $119.1(15)$ & $\mathrm{F} 22-\mathrm{P} 2-\mathrm{F} 21$ & $105.8(12)$ \\
\hline $\mathrm{C} 13-\mathrm{C} 14-\mathrm{H} 14$ & 120.5 & $\mathrm{~F} 26-\mathrm{P} 2-\mathrm{F} 21$ & $91.9(10)$ \\
\hline $\mathrm{C} 15-\mathrm{C} 14-\mathrm{H} 14$ & 120.5 & $\mathrm{~F} 22-\mathrm{P} 2-\mathrm{F} 24$ & $168.3(12)$ \\
\hline $\mathrm{C} 16-\mathrm{C} 15-\mathrm{C} 14$ & $116.9(15)$ & $\mathrm{F} 26-\mathrm{P} 2-\mathrm{F} 24$ & 89.7 (11) \\
\hline $\mathrm{C} 16-\mathrm{C} 15-\mathrm{H} 15$ & 121.5 & $\mathrm{~F} 21-\mathrm{P} 2-\mathrm{F} 24$ & 85.7 (10) \\
\hline $\mathrm{C} 14-\mathrm{C} 15-\mathrm{H} 15$ & 121.5 & $\mathrm{~F} 22-\mathrm{P} 2-\mathrm{F} 23$ & $86.1(11)$ \\
\hline $\mathrm{N} 5-\mathrm{C} 16-\mathrm{C} 15$ & $123.6(12)$ & $\mathrm{F} 26-\mathrm{P} 2-\mathrm{F} 23$ & $90.6(11)$ \\
\hline $\mathrm{N} 5-\mathrm{C} 16-\mathrm{C} 17$ & $116.1(11)$ & $\mathrm{F} 21-\mathrm{P} 2-\mathrm{F} 23$ & $167.8(12)$ \\
\hline $\mathrm{C} 15-\mathrm{C} 16-\mathrm{C} 17$ & $120.3(13)$ & $\mathrm{F} 24-\mathrm{P} 2-\mathrm{F} 23$ & $82.4(10)$ \\
\hline
\end{tabular}


C18-C17-N4

$\mathrm{C} 18-\mathrm{C} 17-\mathrm{C} 16$

N4-C17-C16

$\mathrm{C} 17-\mathrm{C} 18-\mathrm{C} 19$

C17-C18-H18

C19-C18-H18

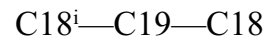

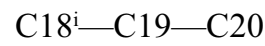

$\mathrm{C} 18-\mathrm{C} 19-\mathrm{C} 20$

$\mathrm{C} 21^{\mathrm{i}}-\mathrm{C} 20-\mathrm{C} 21$

$\mathrm{C} 21^{\mathrm{i}}-\mathrm{C} 20-\mathrm{C} 19$

$\mathrm{C} 21-\mathrm{C} 20-\mathrm{C} 19$

$\mathrm{C} 22-\mathrm{C} 21-\mathrm{C} 20$

$\mathrm{C} 22-\mathrm{C} 21-\mathrm{H} 21$

$\mathrm{C} 20-\mathrm{C} 21-\mathrm{H} 21$

$\mathrm{N} 6-\mathrm{C} 22-\mathrm{C} 21$

$\mathrm{N} 6-\mathrm{C} 22-\mathrm{H} 22$

$\mathrm{C} 21-\mathrm{C} 22-\mathrm{H} 22$

$\mathrm{C} 1-\mathrm{N} 1-\mathrm{C} 5$

C1-N1-Mn1

C5-N1-Mn1

$\mathrm{N} 1-\mathrm{C} 1-\mathrm{C} 2-\mathrm{C} 3$

$\mathrm{C} 1-\mathrm{C} 2-\mathrm{C} 3-\mathrm{C} 4$

$\mathrm{C} 2-\mathrm{C} 3-\mathrm{C} 4-\mathrm{C} 5$

$\mathrm{C} 3-\mathrm{C} 4-\mathrm{C} 5-\mathrm{N} 1$

$\mathrm{C} 3-\mathrm{C} 4-\mathrm{C} 5-\mathrm{C} 6$

$\mathrm{C} 4-\mathrm{C} 5-\mathrm{C} 6-\mathrm{N} 2$

$\mathrm{N} 1-\mathrm{C} 5-\mathrm{C} 6-\mathrm{N} 2$

$\mathrm{C} 4-\mathrm{C} 5-\mathrm{C} 6-\mathrm{C} 7$

$\mathrm{N} 1-\mathrm{C} 5-\mathrm{C} 6-\mathrm{C} 7$

$\mathrm{N} 2-\mathrm{C} 6-\mathrm{C} 7-\mathrm{C} 8$

$\mathrm{C} 5-\mathrm{C} 6-\mathrm{C} 7-\mathrm{C} 8$

$\mathrm{C} 6-\mathrm{C} 7-\mathrm{C} 8-\mathrm{C}^{\mathrm{i}}$

$\mathrm{C} 6-\mathrm{C} 7-\mathrm{C} 8-\mathrm{C} 109$

$\mathrm{C} 6-\mathrm{C} 7-\mathrm{C} 8-\mathrm{C} 209$

$\mathrm{N} 5-\mathrm{C} 12-\mathrm{C} 13-\mathrm{C} 14$

$\mathrm{C} 12-\mathrm{C} 13-\mathrm{C} 14-\mathrm{C} 15$

$\mathrm{C} 13-\mathrm{C} 14-\mathrm{C} 15-\mathrm{C} 16$

$\mathrm{C} 14-\mathrm{C} 15-\mathrm{C} 16-\mathrm{N} 5$

$\mathrm{C} 14-\mathrm{C} 15-\mathrm{C} 16-\mathrm{C} 17$

$\mathrm{N} 5-\mathrm{C} 16-\mathrm{C} 17-\mathrm{C} 18$

$\mathrm{C} 15-\mathrm{C} 16-\mathrm{C} 17-\mathrm{C} 18$

N5-C16-C17-N4

$\mathrm{C} 15-\mathrm{C} 16-\mathrm{C} 17-\mathrm{N} 4$

$\mathrm{N} 4-\mathrm{C} 17-\mathrm{C} 18-\mathrm{C} 19$

$\mathrm{C} 16-\mathrm{C} 17-\mathrm{C} 18-\mathrm{C} 19$

$\mathrm{C} 17-\mathrm{C} 18-\mathrm{C} 19-\mathrm{C} 18^{\mathrm{i}}$
122.1 (12)

125.6 (12)

$112.2(11)$

120.6 (13)

119.7

119.7

116.3 (16)

$121.8(8)$

$121.8(8)$

$115.5(18)$

$122.2(9)$

122.2 (9)

119.7 (15)

120.1

120.1

123.7 (15)

118.1

118.1

117.7 (11)

$123.3(9)$

118.9 (8)

$-2(2)$

1 (2)

-1 (2)

1 (2)

-179.0 (12)

-179.5 (11)

0.1 (15)

1 (2)

-179.5 (12)

0.1 (17)

179.6 (10)

0.0 (8)

$180.0(8)$

180.0 (8)

1 (2)

-1 (2)

-1 (2)

4 (2)

$-177.6(12)$

-172.7 (12)

8.5 (19)

4.9 (14)

$-174.0(10)$

-0.5 (17)

176.9 (10)

0.2 (8)
F22-P2-F25

F26-P2-F25

$\mathrm{F} 21-\mathrm{P} 2-\mathrm{F} 25$

F24-P2-F25

F23-P2-F25

C90-C91-H91A

C90- C91-H91B

H91A-C91-H91B

C90-C91-H91C

H91A-C91-H91C

H91B-C91-H91C

O90- $\mathrm{C} 90-\mathrm{C} 91$

$\mathrm{O} 90-\mathrm{C} 90-\mathrm{C} 92$

$\mathrm{C} 91-\mathrm{C} 90-\mathrm{C} 92$

C90- $992-\mathrm{H} 92 \mathrm{~A}$

C90- $992-\mathrm{H} 92 \mathrm{~B}$

H92A-C92-H92B

C90-C92- $\mathrm{H} 92 \mathrm{C}$

$\mathrm{H} 92 \mathrm{~A}-\mathrm{C} 92-\mathrm{H} 92 \mathrm{C}$

H92B-C92-H92C

$\mathrm{C} 4-\mathrm{C} 5-\mathrm{N} 1-\mathrm{C} 1$

$\mathrm{C} 6-\mathrm{C} 5-\mathrm{N} 1-\mathrm{C} 1$

$\mathrm{C} 4-\mathrm{C} 5-\mathrm{N} 1-\mathrm{Mn} 1$

$\mathrm{C} 6-\mathrm{C} 5-\mathrm{N} 1-\mathrm{Mn} 1$

$\mathrm{C} 7-\mathrm{C} 6-\mathrm{N} 2-\mathrm{C}^{\mathrm{i}}$

$\mathrm{C} 5-\mathrm{C} 6-\mathrm{N} 2-\mathrm{C}^{\mathrm{i}}$

$\mathrm{C} 7-\mathrm{C} 6-\mathrm{N} 2-\mathrm{Mn} 1$

$\mathrm{C} 5-\mathrm{C} 6-\mathrm{N} 2-\mathrm{Mn} 1$

$\mathrm{C} 18-\mathrm{C} 17-\mathrm{N} 4-\mathrm{C} 17^{\mathrm{i}}$

$\mathrm{C} 16-\mathrm{C} 17-\mathrm{N} 4-\mathrm{C} 17^{\mathrm{i}}$

$\mathrm{C} 18-\mathrm{C} 17-\mathrm{N} 4-\mathrm{Mn} 1$

$\mathrm{C} 16-\mathrm{C} 17-\mathrm{N} 4-\mathrm{Mn} 1$

$\mathrm{C} 15-\mathrm{C} 16-\mathrm{N} 5-\mathrm{C} 12$

$\mathrm{C} 17-\mathrm{C} 16-\mathrm{N} 5-\mathrm{C} 12$

$\mathrm{C} 15-\mathrm{C} 16-\mathrm{N} 5-\mathrm{Mn} 1$

$\mathrm{C} 17-\mathrm{C} 16-\mathrm{N} 5-\mathrm{Mn} 1$

$\mathrm{C} 13-\mathrm{C} 12-\mathrm{N} 5-\mathrm{C} 16$

$\mathrm{C} 13-\mathrm{C} 12-\mathrm{N} 5-\mathrm{Mn} 1$

$\mathrm{C} 21-\mathrm{C} 22-\mathrm{N} 6-\mathrm{C} 22^{\mathrm{i}}$

$\mathrm{C} 7 \mathrm{i}-\mathrm{C} 8-\mathrm{C} 109-\mathrm{C} 110$

$\mathrm{C} 7-\mathrm{C} 8-\mathrm{C} 109-\mathrm{C} 110$

$\mathrm{C} 7-\mathrm{C} 8-\mathrm{C} 109-\mathrm{C} 110^{\mathrm{i}}$

$\mathrm{C} 7-\mathrm{C} 8-\mathrm{C} 109-\mathrm{C} 110^{\mathrm{i}}$

$\mathrm{C} 110 \mathrm{i}-\mathrm{C} 109-\mathrm{C} 110-\mathrm{C} 111$

$\mathrm{C} 8-\mathrm{C} 109-\mathrm{C} 110-\mathrm{C} 111$

C109-C110-C111-N13
89.0 (11)

178.5 (12)

87.5 (10)

88.9 (10)

$89.7(10)$

109.5

109.5

109.5

109.5

109.5

109.5

$122(3)$

118 (2)

$120(3)$

109.5

109.5

109.5

109.5

109.5

109.5

-2 (2)

178.3 (11)

179.1 (10)

-0.5 (15)

0.0 (8)

-179.6 (11)

180.0 (8)

0.4 (11)

0.2 (8)

-177.4 (11)

$-179.8(9)$

2.6 (11)

-3.8 (19)

177.4 (10)

169.0 (10)

$-9.8(13)$

1.5 (17)

-170.9 (9)

0.4 (14)

-64.7 (18)

115.3 (18)

115.3 (18)

-64.7 (18)

2 (2)

-178 (2)

-5 (6) 


\begin{tabular}{llll}
$\mathrm{C} 17-\mathrm{C} 18-\mathrm{C} 19-\mathrm{C} 20$ & $-179.8(8)$ & $\mathrm{C} 110-\mathrm{C} 111-\mathrm{N} 13-\mathrm{C} 111^{\mathrm{i}}$ & $3(3)$ \\
$\mathrm{C} 18-\mathrm{C} 19-\mathrm{C} 20-\mathrm{C} 21^{\mathrm{i}}$ & $40.0(9)$ & $\mathrm{C} 7-\mathrm{C} 8-\mathrm{C} 209-\mathrm{C} 210^{\mathrm{i}}$ & $61.4(18)$ \\
$\mathrm{C} 18-\mathrm{C} 19-\mathrm{C} 20-\mathrm{C} 21^{\mathrm{i}}$ & $-140.0(9)$ & $\mathrm{C} 7-\mathrm{C} 8-\mathrm{C} 209-\mathrm{C} 210^{\mathrm{i}}$ & $-118.6(18)$ \\
$\mathrm{C} 18-\mathrm{C} 19-\mathrm{C} 20-\mathrm{C} 21$ & $-140.0(9)$ & $\mathrm{C} 7-\mathrm{C} 8-\mathrm{C} 209-\mathrm{C} 210$ & $-118.6(18)$ \\
$\mathrm{C} 18-\mathrm{C} 19-\mathrm{C} 20-\mathrm{C} 21$ & $40.0(9)$ & $\mathrm{C} 7-\mathrm{C} 8-\mathrm{C} 209-\mathrm{C} 210$ & $61.4(18)$ \\
$\mathrm{C} 21^{\mathrm{i}}-\mathrm{C} 20-\mathrm{C} 21-\mathrm{C} 22$ & $0.4(12)$ & $\mathrm{C} 210^{\mathrm{i}}-\mathrm{C} 209-\mathrm{C} 210-\mathrm{C} 211$ & $-2(3)$ \\
$\mathrm{C} 19-\mathrm{C} 20-\mathrm{C} 21-\mathrm{C} 22$ & $-179.6(12)$ & $\mathrm{C} 8-\mathrm{C} 209-\mathrm{C} 210-\mathrm{C} 211$ & $178(3)$ \\
$\mathrm{C} 20-\mathrm{C} 21-\mathrm{C} 22-\mathrm{N} 6$ & $-1(3)$ & $\mathrm{C} 209-\mathrm{C} 210-\mathrm{C} 211-\mathrm{N} 23$ & $5(6)$ \\
$\mathrm{C} 2-\mathrm{C} 1-\mathrm{N} 1-\mathrm{C} 5$ & $3(2)$ & $\mathrm{C} 210-\mathrm{C} 211-\mathrm{N} 23-\mathrm{C} 211^{\mathrm{i}}$ & $-3(3)$ \\
$\mathrm{C} 2-\mathrm{C} 1-\mathrm{N} 1-\mathrm{Mn} 1$ & $-178.6(10)$ & & \\
\hline
\end{tabular}

Symmetry code: (i) $-x+1, y,-z+3 / 2$.

Hydrogen-bond geometry $\left(A,{ }^{\circ}\right)$

\begin{tabular}{lllll}
\hline$D-\mathrm{H} \cdots A$ & $D-\mathrm{H}$ & $\mathrm{H} \cdots A$ & $D \cdots A$ & $D-\mathrm{H} \cdots A$ \\
\hline $\mathrm{C} 12-\mathrm{H} 12 \cdots \mathrm{F} 25^{\mathrm{ii}}$ & 0.95 & 2.31 & $3.23(3)$ & 162 \\
$\mathrm{C} 15-\mathrm{H} 15 \cdots \mathrm{O} 90^{\mathrm{ii}}$ & 0.95 & 2.58 & $3.53(3)$ & 177 \\
$\mathrm{C} 18-\mathrm{H} 18 \cdots \mathrm{O} 90^{\mathrm{iii}}$ & 0.95 & 2.57 & $3.50(2)$ & 168 \\
$\mathrm{C} 18-\mathrm{H} 18 \cdots \mathrm{O} 90^{\mathrm{ii}}$ & 0.95 & 2.53 & $3.47(2)$ & 169 \\
$\mathrm{C} 22-\mathrm{H} 22 \cdots \mathrm{F} 21^{\mathrm{iv}}$ & 0.95 & 2.47 & $3.42(2)$ & 175
\end{tabular}

Symmetry codes: (ii) $x-1 / 2,-y+3 / 2,-z+1$; (iii) $x-1 / 2, y+1 / 2, z$; (iv) $-x+1,-y+2, z+1 / 2$. 